\title{
Between carbonatite and lamproite-Diamondiferous Torngat ultramafic lamprophyres formed by carbonate-fluxed melting of cratonic MARID-type metasomes
}

\author{
Sebastian Tappe ${ }^{\mathrm{a}, *}$, Stephen F. Foley ${ }^{\mathrm{b}}$, Bruce A. Kjarsgaard ${ }^{\mathrm{c}}$, Rolf L. Romer ${ }^{\mathrm{d}}$, \\ Larry M. Heaman ${ }^{\mathrm{a}}$, Andreas Stracke ${ }^{\mathrm{e}}$, George A. Jenner ${ }^{\mathrm{f}}$ \\ ${ }^{a}$ De Beers Canada Inc., 65, Overlea Boulevard, Suite 300, Toronto, Ont., Canada M4H 1P1 \\ ${ }^{\mathrm{b}}$ Institut für Geowissenschaften, Universität Mainz, Becherweg 21, 55099 Mainz, Germany \\ ${ }^{\mathrm{c}}$ Geological Survey of Canada, Ottawa, Canada K1 A OE8 \\ ${ }^{\mathrm{d}}$ GeoForschungszentrum Potsdam, Telegrafenberg, 14473 Potsdam, Germany \\ ${ }^{\mathrm{e}}$ Institut für Isotopengeologie und Mineralische Rohstoffe, Department of Earth Sciences, \\ ETH-Zürich, Clausiusstrasse 25, CH-8092 Zürich, Switzerland \\ ${ }^{\mathrm{f}}$ Department of Earth Sciences, Memorial University, St. John's, Canada AlB 3 X5
}

Received 12 November 2007; accepted in revised form 11 March 2008; available online 30 March 2008

\begin{abstract}
New U-Pb perovskite ages reveal that diamondiferous ultramafic lamprophyre magmas erupted through the Archean crust of northern Labrador and Quebec (eastern Canada) between ca. 610 and $565 \mathrm{Ma}$, a period of strong rifting activity throughout contiguous Laurentia and Baltica. The observed Torngat carbonate-rich aillikite/carbonatite and carbonate-poor mela-aillikite dyke varieties show a large spread in $\mathrm{Sr}-\mathrm{Nd}-\mathrm{Hf}-\mathrm{Pb}$ isotope ratios with pronounced correlations between isotope systems. An isotopically depleted component is identified solely within aillikites ${ }^{87} \mathrm{Sr} /{ }^{86} \mathrm{Sr}_{\mathrm{i}}=0.70323-0.70377 ; \varepsilon \mathrm{Nd}_{\mathrm{i}}=+1.2-$ $+1.8 ; \varepsilon \mathrm{Hf}_{\mathrm{i}}=+1.4-+3.5 ;{ }^{206} \mathrm{~Pb} /{ }^{204} \mathrm{~Pb}_{\mathrm{i}}=18.2-18.5$ ), whereas some aillikites and all mela-aillikites range to more enriched isotope signatures $\left({ }^{87} \mathrm{Sr} /{ }^{86} \mathrm{Sr}_{\mathrm{i}}=0.70388-0.70523 ; \varepsilon \mathrm{Nd}_{\mathrm{i}}=-0.5\right.$ to $-3.9 ; \varepsilon \mathrm{Hf}_{\mathrm{i}}=-0.6$ to $\left.-6.0 ;{ }^{206} \mathrm{~Pb} /{ }^{204} \mathrm{~Pb}_{\mathrm{i}}=17.8-18.2\right)$. These contrasting isotopic characteristics of aillikites/carbonatites and mela-aillikites, along with subtle differences in their modal carbonate, $\mathrm{SiO}_{2}, \mathrm{Al}_{2} \mathrm{O}_{3}, \mathrm{Na}_{2} \mathrm{O}, \mathrm{Cs}-\mathrm{Rb}$, and $\mathrm{Zr}-\mathrm{Hf}$ contents, are consistent with two distinctive metasomatic assemblages of different age in the mantle magma source region.

Integration of petrologic, geochemical, and isotopic information leads us to propose that the isotopically enriched component originated from a reduced phlogopite-richterite-Ti-oxide dominated source assemblage that is reminiscent of MARID suite xenoliths. In contrast, the isotopically depleted component was derived from a more oxidized phlogopite-carbonate dominated source assemblage. We argue that low-degree $\mathrm{CO}_{2}$-rich potassic silicate melts from the convective upper mantle were preferentially channelled into an older, pre-existing MARID-type vein network at the base of the North Atlantic craton lithosphere, where they froze to form new phlogopite-carbonate dominated veins. Continued stretching and thinning of the cratonic lithosphere during the Late Neoproterozoic remobilized the carbonate-rich vein material and induced volatile-fluxed fusion of the MARID-type veins and the cold peridotite substrate. Isotopic modelling suggests that only $5-12 \%$ trace element contribution from such geochemically extreme MARID-type material is required to produce the observed compositional shift from the isotopically most depleted aillikites/carbonatites towards enriched mela-aillikites.

We conclude that cold cratonic mantle lithosphere can host several generations of contrasting vein assemblages, and that each may have formed during past tectonic and magmatic events under distinctively different physicochemical conditions. Although cratonic MARID-type and carbonate-bearing veins in peridotite can be the respective sources for lamproite and
\end{abstract}

\footnotetext{
* Corresponding author. Fax: +1 416 423-9919.

E-mail address: sebastian.tappe@ca.debeersgroup.com (S. Tappe).
} 
carbonatite magmas when present as the sole metasome, their concomitant fusion in a complex source region may give rise to a whole new variety of deep volatile-rich magmas and we suggest that orangeites (formerly Group 2 kimberlites), kamafugites, and certain types of ultramafic lamprophyre are formed in this manner.

(C) 2008 Published by Elsevier Ltd.

\section{INTRODUCTION}

Carbonatites and lamproites are some of the most extreme partial melting products of mantle materials that have been previously enriched in incompatible elements and volatiles. On the basis of experimental studies they appear to represent opposite ends of the volatile spectrum; carbonatites clearly require abundant $\mathrm{CO}_{2}$ in the source (Wyllie and Huang, 1975; Olafsson and Eggler, 1983; Eggler, 1989; Wyllie, 1989), whereas lamproites require $\mathrm{H}_{2} \mathrm{O}-$ rich sources (Arima and Edgar, 1983b; Foley et al., 1987; Edgar and Vukadinovic, 1992) in which carbon, if present in any abundance at all, probably exists as $\mathrm{CH}_{4}$ (Foley et al., 1986; Foley, 1993). Coupled to these distinctive volatile compositions in the source regions, carbonatites and lamproites each have highly specific characteristics as regards major element composition and incompatible element distribution, indicating contrasting source enrichment styles that resulted in specific metasomatic assemblages (Green and Wallace, 1988; Meen et al., 1989; Foley, 1992a,b; Thibault et al., 1992; Mitchell, 1995; Chakhmouradian, 2006). In this paper we present the case for the concurrent melting of two such contrasting metasomatic assemblages that had been introduced into the North Atlantic craton (NAC) mantle lithosphere at different times. The outcome was a suite of carbonate-potassic silicate magmas, the Torngat ultramafic lamprophyres, which form a diamondiferous dyke swarm across northern Labrador and easternmost Quebec, Canada (Tappe et al., 2004).

The Torngat ultramafic lamprophyre (UML) suite comprises rare dolomite carbonatite and abundant aillikite, which is the carbonate-rich UML variant. Aillikite compositionally grades into carbonate-poor (to carbonate-free) varieties, for which we introduced the term mela-aillikite (Tappe et al., 2004, 2005). Although we previously noted these mela-aillikites can have mineralogical similarities to olivine lamproites, i.e., the occurrence of olivine and phlogopite phenocrysts with groundmass high-Ti potassian richterite, they differ from olivine lamproites in being significantly $\mathrm{SiO}_{2}$-undersaturated (30$37 \mathrm{wt} \%$ as opposed to $41-46 \mathrm{wt}^{\%} \% \mathrm{SiO}_{2}$ for typical olivine lamproites; Mitchell and Bergman, 1991; Foley, 1993). This points to aillikite and, importantly, mela-aillikite magma generation in the presence of $\mathrm{CO}_{2}$ (cf. Green and Falloon, 1998), a volatile species not essential in the genesis of lamproites (Foley et al., 1986). Previously, $\mathrm{CO}_{2}$ degassing during UML magma emplacement was suggested as a possible explanation for the Torngat aillikite/carbonatite and mela-aillikite compositional continuum (Tappe et al., 2004).

In this study we report $\mathrm{U}-\mathrm{Pb}$ perovskite ages, radiogenic $(\mathrm{Sr}-\mathrm{Nd}-\mathrm{Hf}-\mathrm{Pb})$ and stable $(\mathrm{C}-\mathrm{O})$ isotope compositions of the Torngat UML suite complemented by data from newly discovered UML dykes from the northern Labrador coast (Fig. 1). We argue that the UML magmas formed by melting of a young phlogopite-carbonate dominated vein assemblage, which caused carbonate-fluxed fusion of ancient cratonic MARID-type veins and the peridotite substrate. If this mechanism gave rise to the aillikite/ carbonatite and mela-aillikite continuum described here, then it appears that the Torngat case straddles conceptual borderlines between carbonatite and lamproite magma geneses. It may thus provide an alternative perspective on the origin of deep volatile-rich magmas (e.g., orangeites and kamafugites) and the processes that surround alkaline melt production at high pressures.

\section{BACKGROUND}

\subsection{North Atlantic craton, Torngat Mountains, and diamondiferous UML dykes}

Two contrasting Archean domains are juxtaposed in northern Labrador (Fig. 1). The Early Archean Saglek block to the east shares many compositional and structural features with the tonalitic crust of western Greenland and, thus, was assigned to the North Atlantic craton (Bridgwater et al., 1973). The reworked Late Archean block to the west is poorly understood and has been variably assigned to the Churchill (Rae subdomain) or the Superior provinces (Korstgård et al., 1987; Rivers et al., 1996; Wardle et al., 2002). Less uncertain, however, is that these two ancient terranes collided at about 1900 to $1800 \mathrm{Ma}$ (Hoffman, 1990), from then on forming an integral part of the supercraton Laurentia (Hoffman, 1988). The Paleoproterozoic suture zones form high-grade orogenic belts with steep topography, for example the Torngat Mountains of northern Labrador and Quebec (Rivers et al., 1996).

Numerous UML dykes occur in northern Labrador and Quebec following a northwest to northeast trend $\left(300^{\circ}\right.$ to $060^{\circ}$ ). They are undeformed, up to 3-m-wide, steeply dipping $\left(>45^{\circ}\right)$, and individual segments can be traced for several kilometres along strike. Digonnet et al. (2000) reported a ${ }^{40} \mathrm{Ar} /{ }^{39} \mathrm{Ar}$ phlogopite age of $550 \pm 15 \mathrm{Ma}(2 \sigma)$ for an aillikite dyke from Abloviak Fjord in the Torngat Mountains of northern Quebec. Tappe et al. (2004) demonstrated the extension of this UML swarm into Labrador to the southeast and emphasized the compositional diversity. The aillikite/carbonatite and mela-aillikite dyke varieties recognized in the Abloviak Fjord region were collectively referred to as 'Torngat UML' by Tappe et al. (2004), who determined an $\mathrm{U}-\mathrm{Pb}$ perovskite emplacement age of $584.0 \pm 3.6 \mathrm{Ma}$ $(2 \sigma)$ for aillikite dyke Q39. These dykes intruded an area that is at least $1500 \mathrm{~km}^{2}$ in size and, thus, form one of the largest known UML swarms. Some of the Torngat UMLs contain sparse but high-quality diamonds (up to 0.685 carats; Twin 


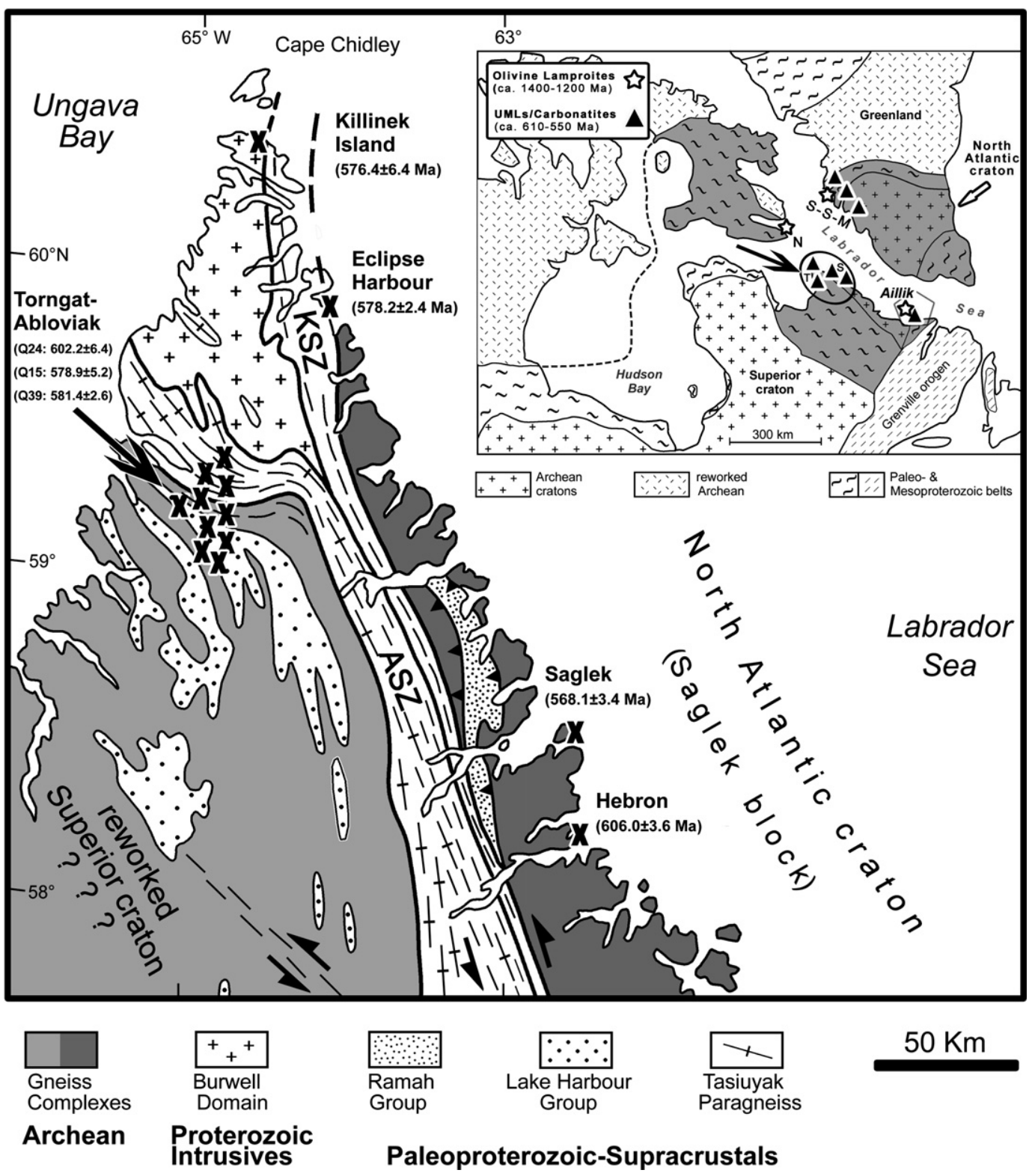

Fig. 1. Simplified geological map of the Torngat Mountains in northern Labrador and Quebec (modified from Wardle et al., 2002). The Torngat and Saglek block UML dyke occurrences investigated during this study are shown as black Xs. The new U-Pb perovskite ages and $2 \sigma$ errors are given in brackets. The inset map provides an overview of the northeastern Canadian-Greenland Shield with grey-shaded areas representing the North Atlantic craton with surrounding mobile belts on either side of the Labrador Sea. Mesoproterozoic olivine lamproite (ca. 1400 to $1200 \mathrm{Ma}$ ) and Neoproterozoic UML/carbonatite (ca. 610 to $550 \mathrm{Ma}$ ) occurrences in the vicinity of the Labrador Sea are also shown. Note that the position of Greenland is restored for Cenozoic continental drift due to now extinct sea-floor spreading in the Labrador Sea (modified from Tappe et al., 2007). Tectonic features and alkaline rock occurrences mentioned in the text are abbreviated as follows: ASZ, Abloviak shear zone; KSZ, Komaktorvik shear zone; N, Napoleon Bay; S, Saglek block; SSM, Sisimiut-Sarfartoq-Maniitsoq areas; T, Torngat Mountains.

Mining Annual Report 2002; http://www.sedar.com). The Torngat samples provide the basis for this isotope study and we have included new UML samples from the northern Labrador coast at Hebron, Saglek, Eclipse Harbour, and Killinek Island in order to examine potential variations among UMLs from different cratonic entities. These four coastal Labrador samples are hereafter collectively referred to as the 'Saglek block UML' (Fig. 1).

\subsection{Aillikite/carbonatite and mela-aillikite compositional continuum}

Aillikite is the carbonate-rich member of the UML group (Rock, 1991; Tappe et al., 2005), which encompasses olivine- and phlogopite-bearing hypabyssal rocks (Fig. 2) derived from a volatile-rich, potassic, $\mathrm{SiO}_{2}$-poor parental magma. In general, aillikite dykes are often found in the 

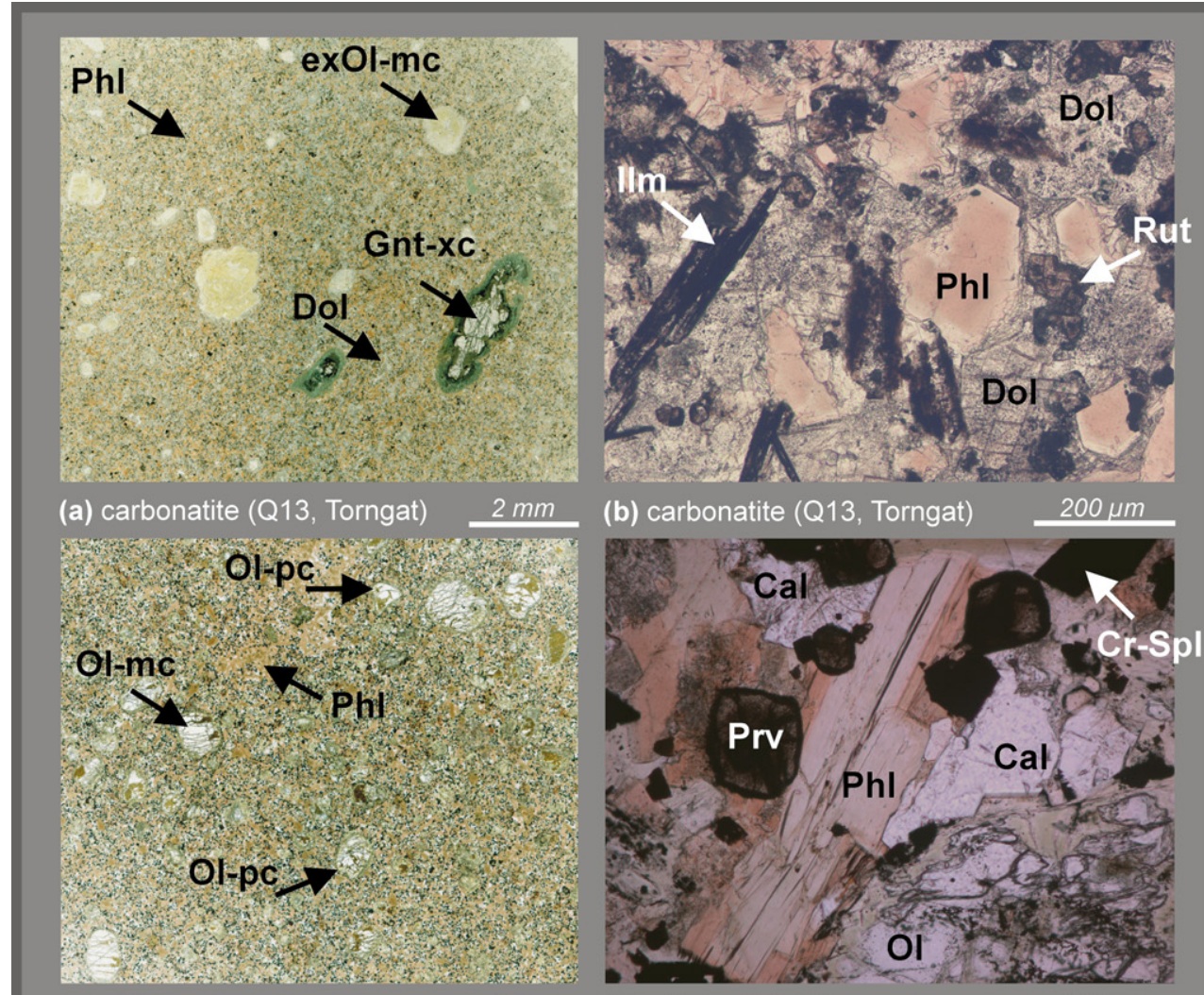

(c) aillikite (Q34, Torngat)
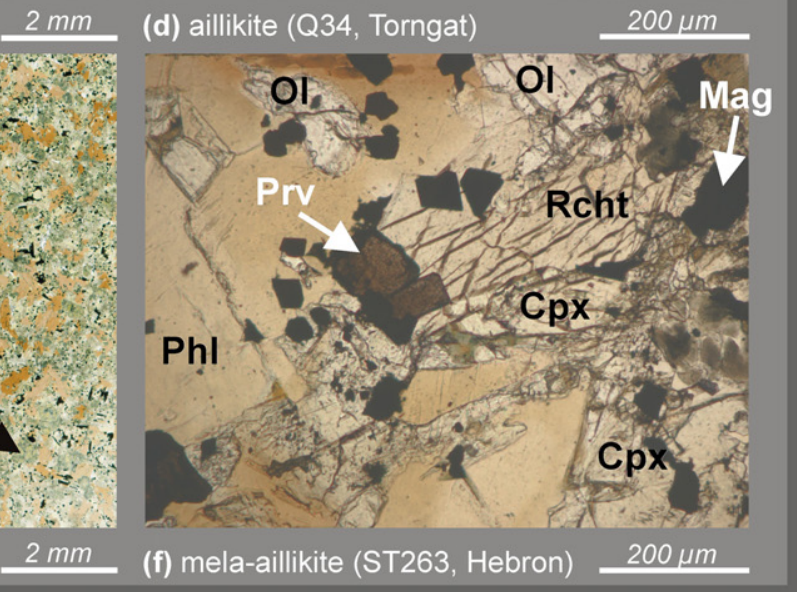

Fig. 2. Overview thin section scans (left) and photomicrographs (plane polarized light, right) of the northern Labrador aillikite/carbonatite and mela-aillikite dyke rocks: (a) Fine-grained dolomite carbonatite Q13 containing few olivine macrocrysts (Ol-mc) and pyrope garnet xenocrysts (Gnt-xc) set in a dolomite and phlogopite dominated matrix. (b) Photomicrograph of dolomite carbonatite Q13 showing the dolomite (Dol) and phlogopite (Phl) dominated matrix plus rutile (Rut) grains and ilmenite (Ilm) laths. (c) Porphyritic aillikite Q34 containing abundant olivine (O1pc) and phlogopite phenocrysts, as well as rare olivine macrocrysts set in a carbonate groundmass.(d) Photomicrograph of aillikite Q34 showing olivine, phlogopite, chromian spinel (Cr-Spl), and perovskite (Prv) microphenocrysts in a calcite (Cal) groundmass. (e) Intergranular melaaillikite Q15 with abundant phlogopite plates, clinopyroxene $(\mathrm{Cpx})$ prisms, and ilmenite laths. Rare carbonate is restricted to the interstices between mafic silicate crystals. (f) Photomicrograph of Hebron mela-aillikite ST263 exhibiting diopside-rich clinopyroxene and titanian magnesiokatophorite-rich richteritic amphibole prisms (Rcht) that are confined to interstitial space between large phlogopite plates. Note furthermore the fresh olivine, magnetite (Mag), and perovskite microphenocrysts that are typically enclosed by phlogopite plates.

vicinity of carbonatite intrusions and can be observed grading into varieties of carbonatite where they have $>50 \mathrm{vol} \%$ modal carbonate. Recently, Mitchell (2005) suggested a lower limit of $30 \mathrm{vol} \%$ carbonate in order to reinforce the genetic link between, for example, some aillikites and car- bonatites. Prime examples of this association are observed at Fen in Scandinavia (e.g., Dahlgren, 1994), Sarfartoq in western Greenland (e.g., Larsen and Rex, 1992; Mitchell et al., 1999), and Aillik Bay in central Labrador (e.g., Tappe et al., 2006). However, regional aillikite dyke swarms also 
occur in areas where there is no indication of central-complex carbonatite intrusions, such as in the Torngat Mountains. In this area, Late Neoproterozoic aillikite/ carbonatite and carbonate-poor mela-aillikite dykes co-exist, and appear to represent a compositional continuum between carbonate-rich and carbonate-free end-members. The following provides background information about the Torngat UMLs summarized from Tappe et al. (2004).

The Torngat mela-aillikites contain significant groundmass clinopyroxene and rare richteritic amphibole instead of abundant primary carbonate (Fig. 2). This reflects lower $\mathrm{CO}_{2}(<5 \mathrm{wt} \%)$, but slightly elevated $\mathrm{SiO}_{2}(30-37.4 \mathrm{wt} \%)$, $\mathrm{Al}_{2} \mathrm{O}_{3}\left(4-6 \mathrm{wt}^{\%} \%\right)$, and $\mathrm{Na}_{2} \mathrm{O}(0.3-0.9 \mathrm{wt} \%)$ contents compared to the aillikite magma $\left(3-22 \mathrm{wt} \% \mathrm{CO}_{2} ; 22-30 \mathrm{wt} \%\right.$ $\mathrm{SiO}_{2} ; 2.2-4.4$ wt $\% \mathrm{Al}_{2} \mathrm{O}_{3} ;<0.3 \mathrm{wt} \% \mathrm{Na}_{2} \mathrm{O}$; Fig. 3). However, Torngat aillikites and mela-aillikites have overlapping high MgO (12-24 wt\%), Ni (229-1110 ppm), and Cr (290$1570 \mathrm{ppm}$ ) contents and, thus, cannot be related by fractional crystallization of spinels and/or common mafic silicate minerals such as olivine. Furthermore, overlapping high $\mathrm{TiO}_{2}(3-10 \mathrm{wt} \%)$ and $\mathrm{K}_{2} \mathrm{O}(1.4-3.5 \mathrm{wt} \%$; Fig. 3) excludes a genetic link between the UML varieties by fractionation or accumulation of Ti-oxides and/or phlogopite. Some aillikites are conspicuously richer in ferric iron than mela-aillikites $\left(0.4-2.3\right.$ vs. $\left.0.7-1.7 \quad \mathrm{Fe}_{2} \mathrm{O}_{3} / \mathrm{FeO}\right)$ at similar bulk-rock total iron contents, implying these magmas record different primary oxidation states (Tappe et al., 2004).

Based on their mineral assemblages and compositions, Tappe et al. (2004) interpreted the Torngat aillikites and mela-aillikites as being derived from a common magma but following different crystallization paths due to $\mathrm{CO}_{2}$ loss, distinctive cooling histories, and variable redox conditions during emplacement. Here, we utilize tracer isotope ratios that allow us to formulate a more robust model in which the aillikite/carbonatite and mela-aillikite compositional continuum is related to the melting process of a common but heterogeneous upper mantle source region.

\section{ANALYTICAL TECHNIQUES}

\section{1. $\mathrm{U}-\mathrm{Pb}$ perovskite geochronology}

Two aillikite and five mela-aillikite hand specimens were processed through standard crushing and mineral separation procedures (Wilfley table, methylene iodide, Frantz isodynamic separator) at the University of Alberta following the techniques described in Heaman and Kjarsgaard (2000). Perovskite recovery was best in $20-100 \mu \mathrm{m}$ range. Fresh euhedral crystals without visible inclusions were individually selected, collected as morphologic fractions and dissolved in a mixture of $\mathrm{HF}$ and $\mathrm{HNO}_{3}$ (typically more than 100 grains per fraction to obtain $>50 \mu$ g aliquots). A ${ }^{235} \mathrm{U}_{-}{ }^{205} \mathrm{~Pb}$ tracer was added to the perovskite/acid mix before dissolution in order to determine $\mathrm{U}$ and $\mathrm{Pb}$ concentrations by isotope dilution. Thorium concentrations were calculated based on the amount of ${ }^{208} \mathrm{~Pb}$ present and the ${ }^{207} \mathrm{~Pb} /{ }^{206} \mathrm{~Pb}$ model age. Uranium and $\mathrm{Pb}$ were isolated and concentrated from perovskite using a $\mathrm{HBr}$ anion exchange chromatography technique. The isotopic composition of these elements was measured on a VG354 thermal ionization mass spectrometer operating in analogue Daly mode. All isotopic data reported in Table 1 were corrected for mass discrimination $(0.105 \% / \mathrm{amu} \mathrm{Pb}$ and $0.123 \% / \mathrm{amu} \mathrm{U})$, tracer, and blank contribution. The presence of initial common lead was corrected using the terrestrial lead evolution model of Stacey and Kramers (1975). The ${ }^{206} \mathrm{~Pb} /{ }^{238} \mathrm{U}$ perovskite ages were shown to be most robust because they are least sensitive to this initial common lead correction (Heaman, 1989; Heaman and Kjarsgaard, 2000).

\subsection{Bulk-rock major and trace element composition}

Rock powders of $<20 \mu \mathrm{m}$ grain size were prepared in an agate planetary ball mill at the Universities of Greifswald and Mainz (Germany) and supplied to Activation Laboratories (Ancaster, Canada) for analyses. Major elements and selected trace elements were determined using a fusion digestion and ICP-AES; and representative analyses have already been published in Tappe et al. (2004). Some samples were additionally analyzed by XRF on fused glass discs at the Universities of Greifswald and Mainz. Results of the two techniques agree within analytical uncertainty. Rare earth elements and other trace elements (e.g., $\mathrm{Nb}, \mathrm{Th}, \mathrm{U}$ ) were analysed using an acid dissolution and ICP-MS. The $\mathrm{U}$ and $\mathrm{Pb}$ concentrations of those samples analyzed for $\mathrm{Pb}$ isotope composition were determined by ID-TIMS in Potsdam (Germany) using a mixed ${ }^{235} \mathrm{U}_{-}{ }^{208} \mathrm{~Pb}$ tracer. Concentrations for elements determined by ICPMS and XRF (e.g., Sr, Ce, Y, Zr, U, Th, and Pb), as well as IDTIMS ( $\mathrm{U}$ and $\mathrm{Pb}$ ) compare within analytical error. Volatiles were determined by a combination of loss on ignition (corrected for the conversion of $\mathrm{FeO}$ to $\mathrm{Fe}_{2} \mathrm{O}_{3}$ ) and direct determination of $\mathrm{CO}_{2}$ using a $\mathrm{C}-\mathrm{S}$ analyser.

\subsection{Bulk-rock $\mathrm{Sr}-\mathrm{Nd}-\mathrm{Hf}-\mathrm{Pb}$ isotope composition}

Isotope compositions were determined on the same bulk-rock powders as the major and trace element contents. Two sample dissolution setups were tested: (1) Powders were loaded together with $\mathrm{HF} / \mathrm{HNO}_{3}$ in microcapsules, which were placed together in an external Teflon-lined steel autoclave, and heated in an oven at $160{ }^{\circ} \mathrm{C}$ for 7 days. (2) Powders were attacked in a $\mathrm{HF} / \mathrm{HNO}_{3}$ mixture in Savillex beakers on a hotplate for 3 days. Subsequently, the $\mathrm{HF} / \mathrm{HNO}_{3}$ mixture from both acid digestion procedures was gently evaporated to incipient sample dryness. The sample material was then taken up in $6 \mathrm{~N} \mathrm{HCl}$ and heated again until a clear solution was obtained; i.e., for 2-3 days. This test indicated complete sample dissolution without bombs, because the measured $\mathrm{Hf}$ isotope composition of duplicate pairs of aillikite Q24 and melaaillikite Q23 are identical within analytical error (Electronic Annex A). Thus, the less tedious powder digestion procedure using Savillex beakers was applied for the remainder of the samples.

$\mathrm{Sr}$ and $\mathrm{Nd}$ were separated and concentrated by standard procedures using Bio-Rad AG50W cation and Eichrom Ln-Spec anion exchange resin, respectively. $\mathrm{Sr}$ and $\mathrm{Nd}$ isotope compositions were measured on a VG 54-30 Sector (Ta single filaments) and Finnigan MAT 262 ( $\operatorname{Re}$ double filaments) thermal ionization mass spectrometer, respectively, in dynamic mode (GFZ Potsdam, Germany). The measured ${ }^{87} \mathrm{Sr} /{ }^{86} \mathrm{Sr}$ ratios were normalized to ${ }^{86} \mathrm{Sr} /{ }^{88} \mathrm{Sr}=0.1194$ and ${ }^{143} \mathrm{Nd} /{ }^{144} \mathrm{Nd}$ to ${ }^{146} \mathrm{Nd} /{ }^{144} \mathrm{Nd}=0.7219$. During the measurement period, the NBS-987 Sr reference material yielded an average value for ${ }^{87} \mathrm{Sr} /{ }^{86} \mathrm{Sr}$ of $0.710270 \pm 14(2 \sigma \mathrm{SD}$ for 18 measurements) and the La Jolla standard yielded a ${ }^{143} \mathrm{Nd} /{ }^{144} \mathrm{Nd}$ value of $0.511850 \pm 7$ ( $2 \sigma$ S.D. for 11 measurements).

Hf was separated using Eichrom Ln-Spec anion exchange resin following the procedure described by Münker et al. (2001). The Hf isotopic composition was analyzed by plasma ionization multicollector mass spectrometry (MC-ICP-MS) on a $N u$ Plasma multicollector ICP-MS instrument in static mode (MPI Mainz, Germany) and all isotope ratios were corrected with an exponential fractionation law using ${ }^{179} \mathrm{Hf} /{ }^{177} \mathrm{Hf}=0.7325$. Mass ${ }^{173} \mathrm{Yb}$ and ${ }^{175} \mathrm{Lu}$ were monitored for isobaric interferences of ${ }^{176} \mathrm{Yb}$ and ${ }^{176} \mathrm{Lu}$ 


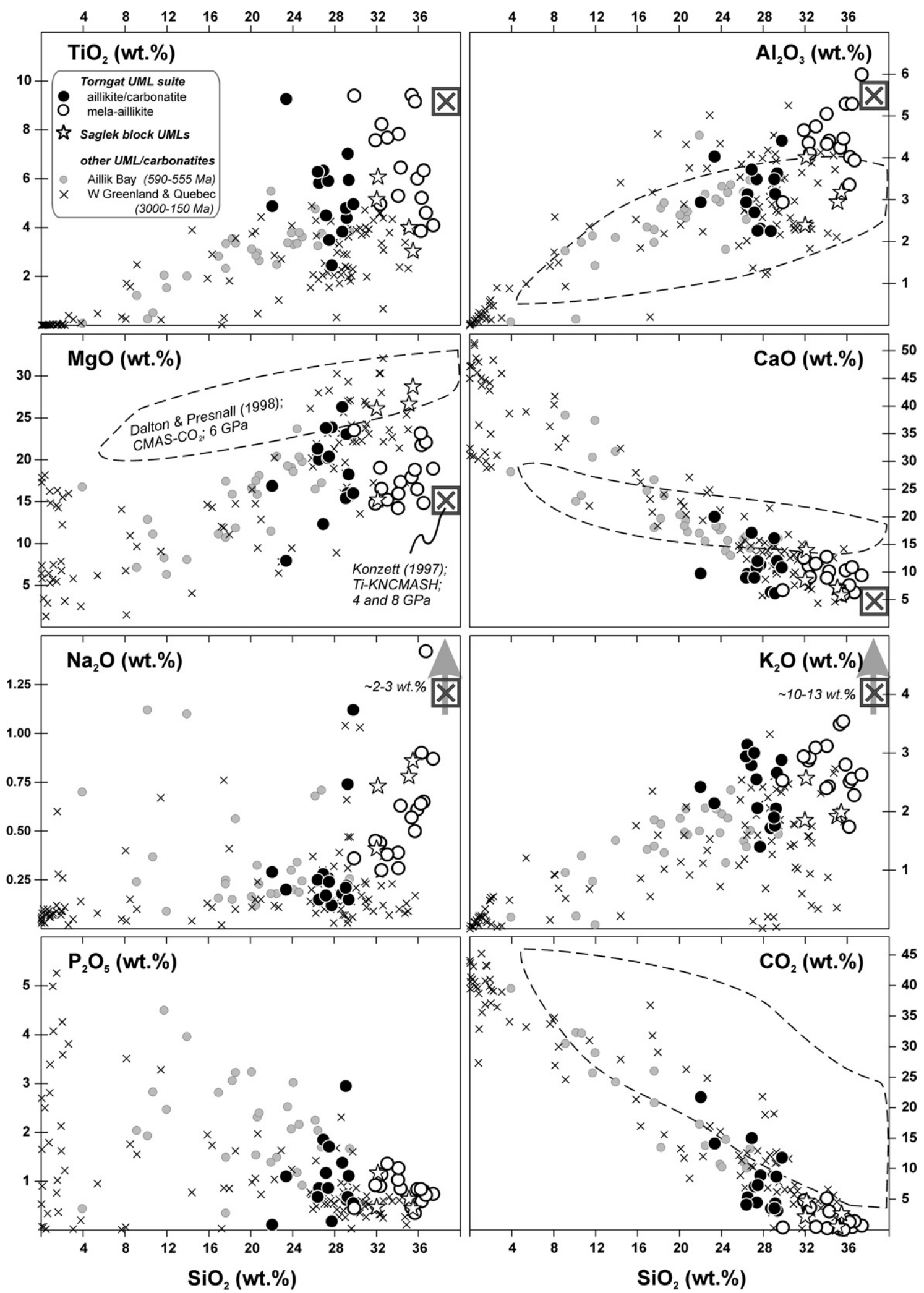

Fig. 3. Major element oxide vs. $\mathrm{SiO}_{2}(\mathrm{wt} \%)$ for the Torngat and Saglek block aillikite/carbonatite and mela-aillikite dykes. The dashed field shows the compositional range of six near-solidus melts including $1 \sigma$ errors produced experimentally from synthetic carbonated peridotite in a simple CMAS- $\mathrm{CO}_{2}$ system at $6 \mathrm{GPa}$ (Dalton and Presnall, 1998). The grey box with the cross shows the area covered by two experimental melt compositions produced from a synthetic MARID-type starting material in a Ti-KNCMASH system at 4 and 8 GPa (Konzett, 1997). Other UML/carbonatite intrusives from central Labrador (Aillik Bay), western Greenland, and eastern Quebec of various ages belonging to the aillikite - carbonatite association are shown for comparison. Data sources are available from the first author upon request. 
Table 1

$\mathrm{U}-\mathrm{Pb}$ perovskite results for ultramafic lamprophyre dykes from the Torngat Mountains and the Saglek block, North Atlantic craton, Labrador, Canada

\begin{tabular}{|c|c|c|c|c|c|c|c|c|c|c|c|c|c|}
\hline \multirow[t]{2}{*}{ Description $^{\mathrm{a}}$} & \multirow{2}{*}{$\begin{array}{l}\text { Weight } \\
(\mu \mathrm{g})\end{array}$} & \multirow{2}{*}{$\begin{array}{l}\mathrm{U} \\
(\mathrm{ppm})\end{array}$} & \multirow{2}{*}{$\begin{array}{l}\text { Th } \\
(\mathrm{ppm})\end{array}$} & \multirow{2}{*}{$\begin{array}{l}\mathrm{Pb} \\
(\mathrm{ppm})\end{array}$} & \multirow[t]{2}{*}{$\mathrm{Th} / \mathrm{U}$} & \multirow{2}{*}{$\begin{array}{l}\mathrm{TCPb} \\
(\mathrm{pg})\end{array}$} & \multirow[t]{2}{*}{${ }^{206} \mathrm{~Pb} /{ }^{238} \mathrm{U}^{\mathrm{b}}$} & \multirow[t]{2}{*}{${ }^{207} \mathrm{~Pb} /{ }^{235} \mathrm{U}^{\mathrm{b}}$} & \multirow[t]{2}{*}{${ }^{207} \mathrm{~Pb} /{ }^{206} \mathrm{~Pb}^{\mathrm{b}}$} & \multicolumn{4}{|c|}{ Apparent ages (Ma) } \\
\hline & & & & & & & & & & ${ }^{206} \mathrm{~Pb} /{ }^{238} \mathrm{U}$ & ${ }^{207} \mathrm{~Pb} /{ }^{235} \mathrm{U}$ & ${ }^{207} \mathrm{~Pb} /{ }^{206} \mathrm{~Pb}$ & $\begin{array}{l}\text { Discordance } \\
{[\%]}\end{array}$ \\
\hline \multicolumn{14}{|l|}{ Torngat Q39 (aillikite) } \\
\hline 1. Dark brown fragments/cubes; M@0.5 A (100) & 38 & 105 & 587 & 29 & 5.6 & 187 & $0.09483 \pm 30$ & $0.7976 \pm 93$ & $0.06101 \pm 68$ & $584.0 \pm 1.8$ & $595.5 \pm 5.3$ & $639 \pm 24$ & 9.1 \\
\hline 2. Dark brown fragments/cubes; M@0.5 A (246) & 50 & 130 & 729 & 36 & 5.6 & 296 & $0.09388 \pm 32$ & $0.7818 \pm 97$ & $0.06040 \pm 74$ & $578.5 \pm 1.9$ & $586.5 \pm 5.6$ & $618 \pm 26$ & 6.7 \\
\hline \multicolumn{14}{|l|}{ Torngat Q24 (aillikite) } \\
\hline Brown clear cubes/octahedrons; M@0.5 A (100) & 105 & 55 & 415 & 25 & 7.6 & 1140 & $0.09792 \pm 55$ & $0.7835 \pm 345$ & $0.05803 \pm 258$ & $602.2 \pm 3.2$ & $588 \pm 20$ & $531 \pm 95$ & -14 \\
\hline \multicolumn{14}{|l|}{ Torngat Q15 (mela-aillikite) } \\
\hline Black irregular fragments; MI@H (40) & 225 & 127 & 1655 & 58 & 13 & 1020 & $0.09395 \pm 43$ & $0.7739 \pm 715$ & $0.05974 \pm 51$ & $578.9 \pm 2.6$ & $582.0 \pm 4.1$ & $594 \pm 19$ & 2.7 \\
\hline \multicolumn{14}{|l|}{ Hebron ST263 (mela-aillikite) } \\
\hline Dark brown irregular fragments; M@0.5 A (152) & 293 & 133 & 150 & 22 & 1.1 & 1850 & $0.09857 \pm 30$ & $0.8064 \pm 977$ & $0.05933 \pm 70$ & $606.0 \pm 1.8$ & $600.4 \pm 5.5$ & $579 \pm 25$ & -4.8 \\
\hline \multicolumn{14}{|l|}{ Saglek ST264 (mela-aillikite) } \\
\hline Black irregular fragments; M@0.3A (150) & 22 & 44 & 1222 & 33 & 28 & 68 & $0.09212 \pm 29$ & $0.7213 \pm 228$ & $0.05679 \pm 172$ & $568.1 \pm 1.7$ & $551 \pm 13$ & $483 \pm 65$ & -18 \\
\hline \multicolumn{14}{|l|}{ Killinek Island ST266 (mela-aillikite) } \\
\hline Brown clear octahedrons/fragments; MI@H (80) & 80 & 52 & 509 & 22 & 9.7 & 371 & $0.09353 \pm 54$ & $0.7767 \pm 162$ & $0.06023 \pm 129$ & $576.4 \pm 3.2$ & $583.6 \pm 9.2$ & $612 \pm 46$ & 6.0 \\
\hline \multicolumn{14}{|l|}{ Eclipse Harbour ST267 (mela-aillikite) } \\
\hline Black irregular fragments; M@0.4A (150) & 63 & 170 & 667 & 35 & 3.9 & 171 & $0.09383 \pm 20$ & $0.7673 \pm 438$ & $0.05930 \pm 31$ & $578.2 \pm 1.2$ & $578.2 \pm 2.5$ & $578 \pm 11$ & 0.0 \\
\hline
\end{tabular}

${ }^{\mathrm{b}}$ Atomic ratios corrected for fractionation, blank (1 pg $\left.\mathrm{Pb} ; 0.5 \mathrm{pg} \mathrm{U}\right)$, isotopic tracer, and initial common $\mathrm{Pb}$. 
on mass ${ }^{176} \mathrm{Hf} .{ }^{176} \mathrm{Lu}$ interferences were negligible and the contribution of ${ }^{176} \mathrm{Yb}$ to the ${ }^{176} \mathrm{Hf}$ signal never exceeded $0.005 \%$ during analysis. The JMC-475 Hf standard was run repeatedly during each analytical session with a mean average for ${ }^{176} \mathrm{Hf} /{ }^{177} \mathrm{Hf}$ of $0.282168 \pm 17(2 \sigma \mathrm{SD}$ for 48 measurements) during the measurement period. This is in good agreement with the long-term average of $0.282161 \pm 16$ ( $2 \sigma \mathrm{SD}$ for 415 measurements).

$\mathrm{Pb}$ from rock powders was separated using anion exchange resin Bio-Rad AG1-X8 applying the $\mathrm{HCl}-\mathrm{HBr}$ technique described by Romer et al. (2005). The Pb isotopic composition was measured on a Finnigan MAT 262 instrument on single Re filaments in static mode (GFZ Potsdam, Germany). Instrumental fractionation was corrected with $+0.1 \%$ per amu as determined from the long-term reproducibility of $\mathrm{Pb}$ reference material NBS-981. Accuracy and precision of reported $\mathrm{Pb}$ isotope ratios is better than $0.1 \%$ at the $2 \sigma$ level of uncertainty.

Total procedural blanks are less than $50 \mathrm{pg} \mathrm{Sr}, 30 \mathrm{pg} \mathrm{Nd}$, $200 \mathrm{pg} \mathrm{Hf}, 30 \mathrm{pg} \mathrm{Pb}$, and considered negligible given the high concentrations of these elements in the samples analysed. The initial $\mathrm{Sr}, \mathrm{Nd}, \mathrm{Hf}$, and $\mathrm{Pb}$ isotopic compositions of the UML dykes were calculated for an intrusion age of $582 \mathrm{Ma}$ (Torngat Mountains), $606 \mathrm{Ma}$ (Hebron), $568 \mathrm{Ma}$ (Saglek), $578 \mathrm{Ma}$ (Eclipse Harbour), and $576 \mathrm{Ma}$ (Killinek Island) using the following decay constants: ${ }^{87} \mathrm{Rb} 1.42 \times 10^{-11} \mathrm{a}^{-1}$ (Steiger and Jäger, 1977); ${ }^{147} \mathrm{Sm}$ $6.54 \times 10^{-12} \mathrm{a}^{-1} \quad$ (Lugmair and Marti, 1978); ${ }^{176} \mathrm{Lu}$ $1.865 \times 10^{-11} \mathrm{a}^{-1}$ (Scherer et al., 2001); ${ }^{232} \mathrm{Th} 4.9475 \times 10^{-11} \mathrm{a}^{-1}$; ${ }^{235} \mathrm{U} 9.8485 \times 10^{-10} \mathrm{a}^{-1} ;{ }^{238} \mathrm{U} 1.55125 \times 10^{-10} \mathrm{a}^{-1}$ (Jaffey et al., 1971; Steiger and Jäger, 1977).

\subsection{Carbon and oxygen isotope composition}

The carbon and oxygen isotope composition of bulk-rock carbonate fractions was measured at the University of Alberta, Canada. Rock powders were reacted with anhydrous $\mathrm{H}_{3} \mathrm{PO}_{4}$ under vacuum at $25{ }^{\circ} \mathrm{C}$ for ca. $24 \mathrm{~h}$ in order to liberate $\mathrm{CO}_{2} . \mathrm{CO}_{2}$ gas recoveries were close to $100 \%$ of theoretical yields so no isotope fractionation during dissolution should have occurred. We applied acid fractionation factors of 1.01025 and 1.01178 to calcite- and dolomite-bearing samples, respectively. The purified $\mathrm{CO}_{2}$ was analysed using a Finnigan MAT-252 gas source mass spectrometer and measured isotope ratios are expressed as $\delta^{13} \mathrm{C}$ and $\delta^{18} \mathrm{O} \%$ relative to PDB (Pee Dee Belemnite) and SMOW (Standard Mean Ocean Water), respectively. Reproducibility was better than $0.1 \%$ for $\delta^{13} \mathrm{C}$ and $0.2 \%$ for $\delta^{18} \mathrm{O}$ as determined by repeated measurements $(n=9,2 \sigma \mathrm{SD})$ of an in-house limestone standard.

\section{NEW U-PB PEROVSKITE AGES}

$\mathrm{U}-\mathrm{Pb}$ perovskite ages were determined for seven individual UML dykes from the Torngat Mountains and the Saglek block along the western NAC edge (Fig. 1). Data are reported in Table 1 and illustrated in Fig. 4. In general, the uranium contents (44-170 ppm) and $\mathrm{Th} / \mathrm{U}$ ratios (1.113.1) of perovskite isolated from these UML dykes are similar to perovskite from North American kimberlites (Heaman and Kjarsgaard, 2000). However, perovskite from the Saglek mela-aillikite ST264 has a higher than average $\mathrm{Th} / \mathrm{U}$ ratio of 27.5 .

A second perovskite fraction from the previously dated Torngat aillikite dyke Q39 (Tappe et al., 2004) was analyzed during this study and yielded a ${ }^{206} \mathrm{~Pb} /{ }^{238} \mathrm{U}$ age of $578.5 \pm 3.8 \mathrm{Ma}$, which is similar to the published age of $584.0 \pm 3.6 \mathrm{Ma}$ (errors are quoted at the $2 \sigma$ level of uncertainty throughout the text). Hence, a weighted average ${ }^{206} \mathrm{~Pb} /{ }^{238} \mathrm{U}$ age of $581.4 \pm 2.6 \mathrm{Ma}$ is considered the best estimate for emplacement of this aillikite dyke. Perovskite from Torngat mela-aillikite dyke Q15 yielded a similar ${ }^{206} \mathrm{~Pb} /{ }^{238} \mathrm{U}$ age of $578.9 \pm 5.2 \mathrm{Ma}$. The age of Torngat aillikite dyke Q24 is slightly older at $602.2 \pm 6.4 \mathrm{Ma}$ and represents the oldest emplacement age yet determined from the Torngat UML dyke swarm. If only these U-Pb age determinations are taken into account, then the Torngat aillik-

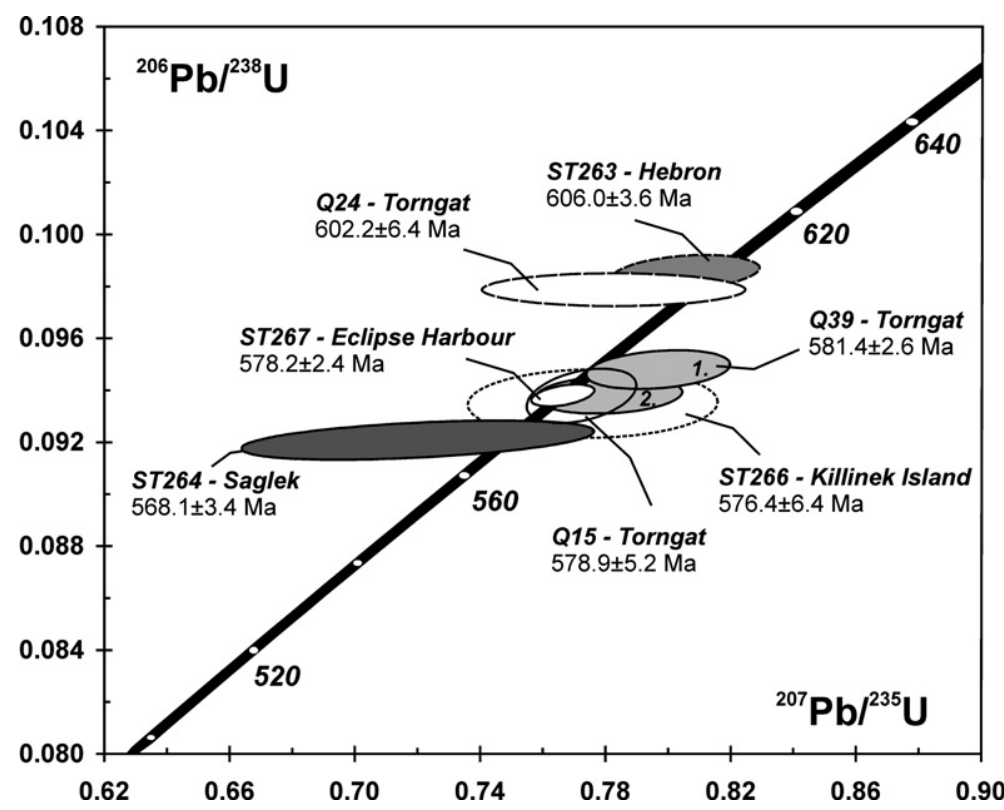

Fig. 4. U-Pb perovskite results for aillikite and mela-aillikite dykes from the Torngat Mountains and the Saglek block in northern Labrador displayed in a concordia diagram. Reported ages are ${ }^{206} \mathrm{~Pb} /{ }^{238} \mathrm{U}$ dates with quoted uncertainties and envelopes at $2 \sigma$. For Torngat aillikite Q39 a weighted average ${ }^{206} \mathrm{~Pb} /{ }^{238} \mathrm{U}$ date of two perovskite fractions is given (1 and 2). See Fig. 1 and Table 1, and text for further details. 
ite/cabonatite and mela-aillikite magmatism occurred over a 20-Myr time span between ca. 600 and $580 \mathrm{Ma}$. However, the ${ }^{40} \mathrm{Ar} /{ }^{39} \mathrm{Ar}$ phlogopite age of $550 \pm 15 \mathrm{Ma}$ determined for a Torngat aillikite dyke by Digonnet et al. (2000) suggests that deep volatile-rich magmatism continued for an even longer time period.

On the Saglek block, the Hebron mela-aillikite dyke ST263 yielded the oldest ${ }^{206} \mathrm{~Pb} /{ }^{238} \mathrm{U}$ perovskite age $(606.0 \pm 3.6 \mathrm{Ma})$ of the UML dykes investigated from the northern Labrador coast, which is similar to the emplacement age of Torngat aillikite Q24. The Eclipse Harbour ST267 (578.2 $\pm 2.4 \mathrm{Ma})$ and Killinek Island ST266 $(576.4 \pm 6.4 \mathrm{Ma})$ mela-aillikite dykes have identical ${ }^{206} \mathrm{~Pb} /{ }^{238} \mathrm{U}$ emplacement ages similar to Torngat UML Q15 and Q39. The Saglek mela-aillikite ST264 $(568.1 \pm 3.4 \mathrm{Ma})$ is the youngest UML dyke known from the northern Labrador coast with an emplacement age that straddles the uncertainty limit of the 'young' ${ }^{40} \mathrm{Ar} /{ }^{39} \mathrm{Ar}$ age for the Torngat aillikite reported by Digonnet et al. (2000).

Taken together, the new ${ }^{206} \mathrm{~Pb} /{ }^{238} \mathrm{U}$ perovskite ages obtained for aillikite and mela-aillikite dykes from the Torngat Mountains and Saglek block indicate that the entire area was subjected to carbonate-potassic silicate magma production during the Late Neoproterozoic between ca. 610 and $565 \mathrm{Ma}$. These currently available age data, however, do not allow better resolution of the timing of UML/carbonatite magmatism within this area. Considering the region covered in Fig. 1, time equivalents occur in the Aillik Bay area, central Labrador coast (ca. 590 to $555 \mathrm{Ma}$; Tappe et al., 2006) and in the Sisimiut-Sarfartoq-Maniitsoq areas of West Greenland (ca. 610 to $550 \mathrm{Ma}$; Larsen and Rex, 1992; Heaman, 2005) at the southern and northern NAC margins, respectively. In the greater North Atlantic region, Late Neoproterozoic UML/carbonatite occurrences are known from the St. Lawrence Valley Rift system (e.g., Doig and Barton, 1968; Gittins et al., 1975) and the Scandinavian Peninsula (e.g., Doig, 1970; Brueckner and Rex, 1980; Dahlgren, 1994).

\section{MAGMA COMPOSITIONS}

Major and compatible trace element compositions of the Torngat UML are summarized in Section 2.2 and Fig. 3; more detailed descriptions can be found in Tappe et al. (2004). The new mela-aillikite samples from the Saglek block compositionally resemble their Torngat analogues and mineralogical descriptions are provided as Electronic annex B. All bulk-rock chemical analyses for the Torngat and Saglek block UML are given in Table 2. The bulk-rock $\mathrm{Sr}-\mathrm{Nd}-\mathrm{Hf}-\mathrm{Pb}$ as well as carbon and oxygen isotope compositions are listed in Table 3. The complete dataset is available online as Electronic annex A.

\subsection{Incompatible elements}

Torngat aillikites/carbonatites and mela-aillikites, as well as Saglek block mela-aillikites have high incompatible element abundances with similar primitive upper mantle (PUM; Palme and O’Neill, 2003) normalized element distri- butions (Fig. 5). They resemble the carbonate-rich type aillikites from Aillik Bay, central Labrador (Tappe et al., 2006, 2007). However, a few subtle but petrogenetically important differences in the incompatible element distribution exist between Torngat aillikites and mela-aillikites on the one hand, and between the Torngat UML and the type rocks from Aillik Bay on the other hand (Fig. 5).

In general, the LFSE, HFSE, and LREE show high concentration levels of up to $700 \times$ PUM, whereas the HREE content is low (below $5 \times \mathrm{PUM}$ for $\mathrm{Yb}$ and $\mathrm{Lu}$ ). The LREE/HREE fractionation is extreme in both Torngat aillikites and mela-aillikites with chondrite normalized La/ $\mathrm{Yb}_{\mathrm{CN}}$ ranging between 70 and 180 . Pronounced relative depletions occur at $\mathrm{K}, \mathrm{Pb}, \mathrm{Sr}-\mathrm{P}$, and $\mathrm{Zr}-\mathrm{Hf}$ in a $\mathrm{PUM}$-normalized multi-element diagram, mainly due to the extreme enrichment of similarly incompatible $\mathrm{Nb}-\mathrm{Ta}$ and the LREE (Fig. 5). A minor trough exists at $\mathrm{U}$, which is in contrast to the $U$ spike in the Aillik Bay pattern.

Torngat aillikites have slightly higher $\mathrm{Cs}$ but lower $\mathrm{Rb}$ concentrations (lower Rb/Cs; Figs. 6 and 7) at a given $\mathrm{K}$ content compared to the majority of mela-aillikites. The characteristic $\mathrm{Zr}-\mathrm{Hf}$ trough in the aillikite/carbonatite incompatible element pattern $\left(\mathrm{ZrHf}^{*}=0.1-0.6\right.$; $\mathrm{ZrHf}^{*} \mathrm{de}-$ fined as PUM-normalized $(\mathrm{Zr}+\mathrm{Hf}) /(\mathrm{Nd}+\mathrm{Sm}))$ is absent from some of the most Ti-rich Torngat and Saglek block mela-aillikites, which have $\mathrm{ZrHf}^{*}$ greater than 0.7 (Figs. 5 and 6). This marked $\mathrm{Zr}-\mathrm{Hf}$ increase at only slightly increasing $\mathrm{Nb}-\mathrm{Ta}$ and constant LREE content from aillikites/carbonatites towards carbonate-poor mela-aillikites is furthermore evident from elevated $\mathrm{Zr} / \mathrm{Nb}, \mathrm{Zr} / \mathrm{Nd}$, and $\mathrm{Zr} /$ $\mathrm{Sm}$ ratios of the mela-aillikites (Fig. 7). The highest $\mathrm{ZrHf}^{*}$ samples have also elevated Rb/Cs (Fig. 6). The Zr/Hf (31$47)$ and $\mathrm{Nb} / \mathrm{Ta}(8-18)$ ratios are highly variable; however, no difference is apparent between aillikites and mela-aillikites (Fig. 8). These ratios overlap the range reported for oceanic basalts (cf. Pfänder et al., 2007). Mela-aillikites tend to have slightly higher $\mathrm{Nb} / \mathrm{U}$ than the majority of aillikites ( $35-55$ vs. $23-43$; Fig. 7). Importantly, all Nb/U values are significantly higher than for average continental crust (ca. 8-12; Rudnick and Fountain, 1995) and fall within, or close to, the range of oceanic basalts $(47 \pm 10$; Hofmann et al., 1986).

\section{2. $\mathrm{Sr}-\mathrm{Nd}-\mathrm{Hf}-\mathrm{Pb}$ isotope composition}

The Torngat aillikites/carbonatites and mela-aillikites define an array in $\mathrm{Sr}-\mathrm{Nd}-\mathrm{Hf}$ isotope space between isotopically depleted and enriched end-members (Figs. 9 and 10). Aillikites/carbonatites show a wide range of initial ${ }^{87} \mathrm{Sr} /{ }^{86} \mathrm{Sr}$ $(0.70323-0.70545 ; \quad \mathrm{Q} 35=0.70713), \varepsilon_{\mathrm{Nd}}(-3.1$ to +1.8 ; $\mathrm{Q} 35=-4.9)$, and $\varepsilon_{\mathrm{Hf}}(-4.8$ to $+3.5 ; \mathrm{Q} 35=-5.7)$ values. Although overlapping to a large extent, mela-aillikites have a more restricted, long-term enriched radiogenic isotope composition characterized by negative $\varepsilon_{\mathrm{Nd}(\mathrm{i})}(-3.9$ to $-0.5)$ and $\varepsilon_{\mathrm{Hf}(\mathrm{i})}(-6.0$ to -0.6$)$ at moderately radiogenic ${ }^{87} \mathrm{Sr} /{ }^{86} \mathrm{Sr}_{(\mathrm{i})}(0.70388-0.70523)$. Aillikite samples with positive $\varepsilon_{\mathrm{Nd}}$ and $\varepsilon_{\mathrm{Hf}}$ have higher $\mathrm{Fe}_{2} \mathrm{O}_{3} / \mathrm{FeO}$ ratios $(>1.7)$ than the remainder of the Torngat UML suite with negative $\varepsilon_{\mathrm{Nd}}$ and $\varepsilon_{\mathrm{Hf}}$ (Fig. 7e). The Saglek block mela-aillikites fall along the Torngat $\mathrm{Sr}-\mathrm{Nd}-\mathrm{Hf}$ isotope array. Mela-aillikites 


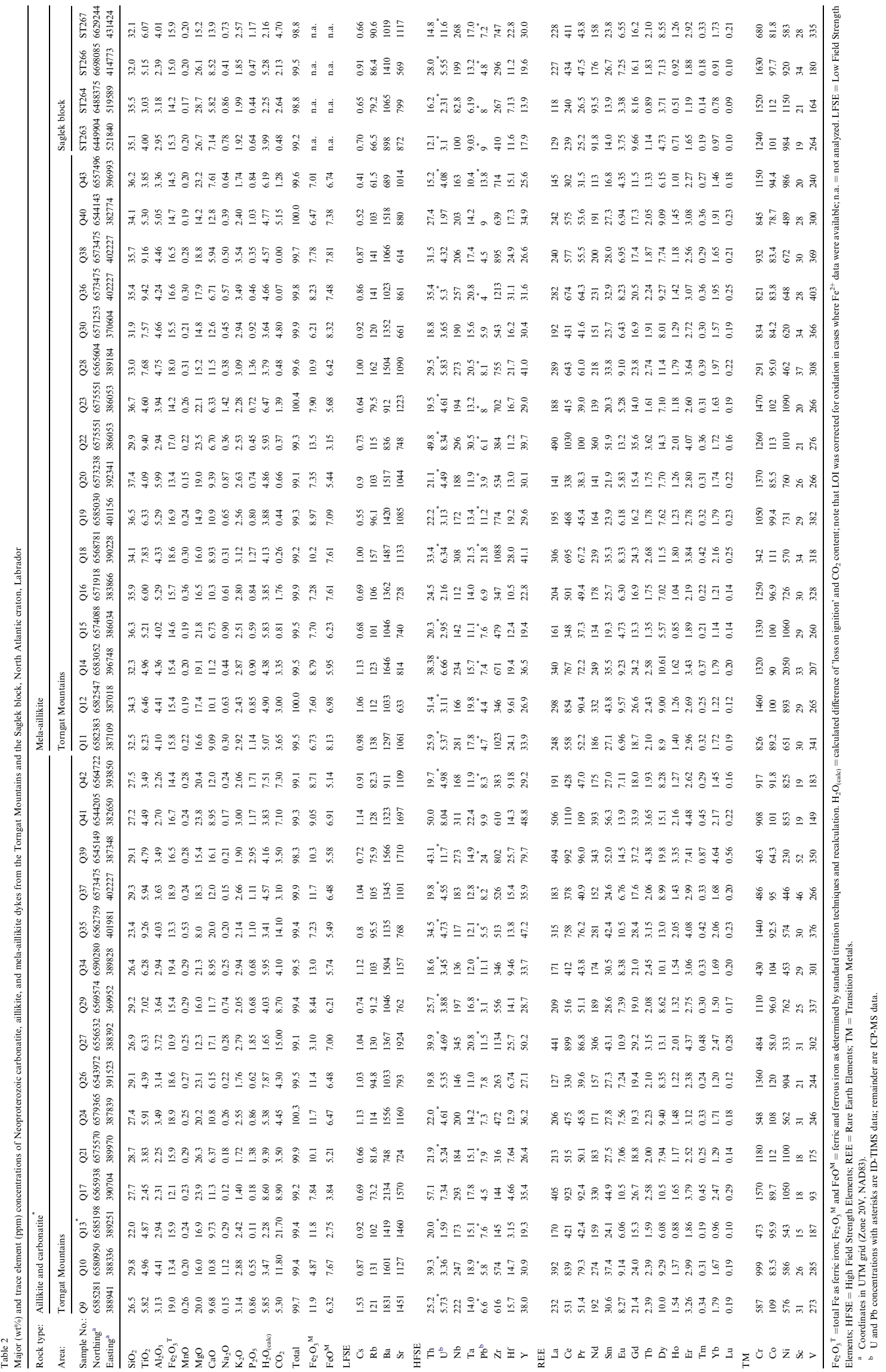


Table 3

$\mathrm{Sr}-\mathrm{Nd}-\mathrm{Hf}-\mathrm{Pb}$ and $\mathrm{C}-\mathrm{O}$ isotope composition of Neoproterozoic carbonatite, aillikite, and mela-aillikite dykes from the Torngat Mountains and the Saglek block, North Atlantic craton, Labrador

\begin{tabular}{|c|c|c|c|c|c|c|c|c|c|c|c|c|c|c|c|c|c|c|}
\hline \multirow{2}{*}{\multicolumn{2}{|c|}{$\frac{{ }^{87} \mathrm{Sr} /{ }^{86} \mathrm{Sr}_{\mathrm{m}}}{\text { Tornoat carbonatite }}$}} & \multicolumn{5}{|c|}{${ }^{\mathrm{a} 87} \mathrm{Sr} /{ }^{86} \mathrm{Sr}_{\mathrm{i}}{ }^{143} \mathrm{Nd} /{ }^{144} \mathrm{Nd}_{\mathrm{m}}{ }^{\mathrm{a} 143} \mathrm{Nd} /{ }^{144} \mathrm{Nd}_{\mathrm{i}}{ }^{\mathrm{b}}\left(\varepsilon_{\mathrm{Nd}}\right)_{\mathrm{i}}{ }^{\mathrm{c}} T_{(\mathrm{DM})}^{\mathrm{Nd}}$} & \multicolumn{4}{|c|}{${ }^{176} \mathrm{Hf} /{ }^{177} \mathrm{Hf} f_{\mathrm{m}}{ }^{\mathrm{a} 176} \mathrm{Hf} /{ }^{177} \mathrm{Hf}_{\mathrm{i}}{ }^{\mathrm{b}}\left(\varepsilon_{\mathrm{Hf}}\right)_{\mathrm{i}}{ }^{\mathrm{c}} T_{(\mathrm{DM})}^{\mathrm{Hf}}$} & \multicolumn{8}{|l|}{${ }^{206} \mathrm{~Pb} / 204$} \\
\hline & & & & & & & & & & & & & & & & & & \\
\hline Q13 & $0.705230(7)$ & 0.70359 & $0.512314(7)$ & 0.51196 & 1.5 & 1.0 & $0.282617(40)$ & 0.28257 & 3.5 & 1.0 & 19.73 & 18.37 & 15.74 & 15.65 & 43.35 & 37.88 & $-3.22(2)$ & $12.91(10)$ \\
\hline \multicolumn{19}{|c|}{ Torngat aillikites } \\
\hline Q9 & $0.705395(7)$ & 0.70344 & $0.512338(6)$ & 0.51197 & 1.6 & 1.0 & $0.282530(6)$ & 0.28251 & 1.5 & 1.0 & 24.79 & 18.44 & 15.99 & 15.62 & 47.65 & 38.74 & $-5.26(9)$ & $9.49(37)$ \\
\hline Q10 & $0.707266(7)$ & 0.70455 & $0.512084(6)$ & 0.51177 & -2.3 & 1.2 & n.a. & n.a. & n.a. & n.a. & 22.39 & 17.94 & 15.87 & 15.60 & 55.06 & 38.39 & $-6.81(1)$ & $13.79(2)$ \\
\hline Q17 & $0.704867(7)$ & 0.70377 & $0.512276(6)$ & 0.51196 & 1.4 & 1.0 & $0.282602(6)$ & 0.28251 & 1.4 & 1.1 & n.a. & n.a. & n.a. & n.a. & n.a. & n.a. & $-4.29(1)$ & $7.86(2)$ \\
\hline Q21 & $0.706955(7)$ & 0.70431 & $0.512088(6)$ & 0.51174 & -2.9 & 1.2 & $0.282379(7)$ & 0.28235 & -4.2 & 1.3 & 22.45 & 17.79 & 15.81 & 15.54 & 46.74 & 40.52 & $-4.84(1)$ & $11.51(1)$ \\
\hline Q24 & $0.705673(8)$ & 0.70337 & $0.512336(5)$ & 0.51196 & 1.4 & 1.0 & $0.282540(5)$ & 0.28252 & 1.8 & 1.0 & 22.72 & 18.33 & 15.89 & 15.63 & 45.33 & 38.65 & $-5.23(4)$ & $11.21(17)$ \\
\hline Q26 & $0.706031(8)$ & 0.70323 & $0.512352(5)$ & 0.51195 & 1.2 & 1.0 & $0.282553(11)$ & 0.28253 & 2.0 & 1.0 & n.a. & n.a. & n.a. & n.a. & n.a. & n.a. & $-4.76(1)$ & $12.18(3)$ \\
\hline Q27 & $0.707032(7)$ & 0.70545 & $0.512078(5)$ & 0.51175 & -2.7 & 1.2 & n.a. & n.a. & n.a. & n.a. & 20.70 & 17.92 & 15.77 & 15.60 & 45.92 & 38.37 & $-5.43(1)$ & $13.71(2)$ \\
\hline Q29 & $0.707371(7)$ & 0.70457 & $0.512160(7)$ & 0.51181 & -1.5 & 1.2 & $0.282428(8)$ & 0.28241 & -2.1 & 1.2 & 29.75 & 18.14 & 16.23 & 15.54 & 64.71 & 40.16 & n.a. & n.a. \\
\hline Q34 & $0.705760(7)$ & 0.70366 & $0.512384(6)$ & 0.51198 & 1.8 & 1.0 & $0.282558(6)$ & 0.28253 & 2.0 & 1.0 & 20.19 & 18.19 & 15.72 & 15.61 & 41.84 & 38.39 & $-5.72(5)$ & $12.80(21)$ \\
\hline Q35 & $0.710044(9)$ & 0.70713 & $0.511984(6)$ & 0.51164 & -4.9 & 1.4 & $0.282334(5)$ & 0.28231 & -5.7 & 1.4 & 24.71 & 17.81 & 15.95 & 15.54 & 56.53 & 40.44 & $-4.94(1)$ & $8.45(1)$ \\
\hline Q37 & $0.705740(7)$ & 0.70349 & $0.512331(6)$ & 0.51196 & 1.4 & 1.0 & $0.282520(5)$ & 0.28251 & 1.4 & 1.0 & 22.29 & 18.48 & 15.86 & 15.63 & 44.53 & 39.22 & $-5.47(1)$ & $13.78(3)$ \\
\hline Q39 & $0.705323(7)$ & 0.70428 & $0.512102(4)$ & 0.51175 & -2.7 & 1.2 & $0.282359(4)$ & 0.28233 & -4.8 & 1.3 & 21.34 & 18.10 & 15.84 & 15.65 & 43.37 & 39.54 & $-5.15(2)$ & $9.26(2)$ \\
\hline Q41 & $0.706181(7)$ & 0.70441 & $0.512061(6)$ & 0.51173 & -3.1 & 1.2 & $0.282360(12)$ & 0.28234 & -4.7 & 1.3 & n.a. & n.a. & n.a. & n.a. & n.a. & n.a. & $-4.44(1)$ & $11.90(2)$ \\
\hline Q42 & $0.706161(7)$ & 0.70442 & $0.512101(5)$ & 0.51174 & -2.8 & 1.2 & $0.282371(5)$ & 0.28234 & -4.5 & 1.3 & 22.22 & 17.98 & 15.83 & 15.58 & 47.63 & 42.26 & $-4.40(2)$ & $13.10(3)$ \\
\hline \multicolumn{19}{|c|}{ Torngat mela-aillikites } \\
\hline Q11 & $0.707583(9)$ & 0.70452 & $0.512068(4)$ & 0.51173 & -3.1 & 1.2 & n.a. & n.a. & n.a. & n.a. & 26.65 & 17.76 & 16.12 & 15.59 & 51.44 & 37.74 & $-6.88(6)$ & $12.84(12)$ \\
\hline Q12 & $0.708768(7)$ & 0.70463 & $0.512038(6)$ & 0.51173 & -3.0 & 1.2 & $0.282335(10)$ & 0.28232 & -5.4 & 1.3 & 23.51 & 17.82 & 15.92 & 15.58 & 58.15 & 28.07 & $-5.94(3)$ & $14.44(7)$ \\
\hline Q14 & $0.707425(7)$ & 0.70388 & $0.512160(6)$ & 0.51183 & -1.1 & 1.1 & $0.282416(9)$ & 0.28240 & -2.5 & 1.2 & 24.77 & 17.94 & 16.01 & 15.61 & 50.50 & 37.92 & $-5.71(1)$ & $13.35(3)$ \\
\hline Q15 & $0.707588(7)$ & 0.70437 & $0.512051(8)$ & 0.51172 & -3.3 & 1.2 & $0.282331(6)$ & 0.28231 & -5.5 & 1.3 & 20.77 & 18.12 & 15.77 & 15.61 & 45.98 & 40.14 & $-6.56(2)$ & $14.30(4)$ \\
\hline Q16 & $0.707841(7)$ & 0.70443 & $0.512049(4)$ & 0.51172 & -3.4 & 1.2 & $0.282320(13)$ & 0.28230 & -6.0 & 1.4 & n.a. & n.a. & n.a. & n.a. & n.a. & n.a. & $-5.47(4)$ & $12.41(6)$ \\
\hline Q18 & $0.707655(7)$ & 0.70440 & $0.512126(7)$ & 0.51178 & -2.0 & 1.2 & $0.282409(5)$ & 0.28240 & -2.6 & 1.2 & 19.74 & 17.88 & 15.69 & 15.58 & 41.60 & 38.48 & n.a. & n.a. \\
\hline Q19 & $0.706393(7)$ & 0.70432 & $0.512023(7)$ & 0.51169 & -3.9 & 1.3 & n.a. & n.a. & n.a. & n.a. & 19.76 & 17.94 & 15.73 & 15.63 & 42.90 & 38.79 & n.a. & n.a. \\
\hline Q20 & $0.706278(7)$ & 0.70397 & $0.512222(5)$ & 0.51186 & -0.5 & 1.1 & $0.282477(10)$ & 0.28245 & -0.6 & 1.1 & 27.13 & 18.20 & 16.10 & 15.57 & 50.76 & 37.32 & $-6.32(1)$ & $14.93(5)$ \\
\hline Q22 & $0.707938(7)$ & 0.70433 & $0.512063(4)$ & 0.51173 & -3.1 & 1.2 & $0.282350(6)$ & 0.28233 & -5.0 & 1.3 & 30.32 & 17.99 & 16.33 & 15.60 & 62.69 & 39.16 & n.a. & n.a. \\
\hline Q23 & $0.706085(7)$ & 0.70453 & $0.512070(5)$ & 0.51173 & -3.2 & 1.2 & $0.282341(5)$ & 0.28232 & -5.2 & 1.3 & 21.96 & 18.00 & 15.82 & 15.59 & 44.28 & 38.99 & $-6.13(6)$ & $14.38(4)$ \\
\hline Q28 & $0.707876(8)$ & 0.70439 & $0.512121(5)$ & 0.51176 & -2.4 & 1.2 & $0.282392(4)$ & 0.28238 & -3.3 & 1.2 & 23.34 & 18.00 & 15.85 & 15.53 & 50.51 & 41.90 & $-5.27(1)$ & 16.11(2) \\
\hline $\mathrm{Q} 30$ & $0.709492(6)$ & 0.70523 & $0.512174(6)$ & 0.51181 & -1.5 & 1.2 & $0.282415(3)$ & 0.28240 & -2.6 & 1.2 & n.a. & n.a. & n.a. & n.a. & n.a. & n.a. & $-4.92(2)$ & $15.25(3)$ \\
\hline Q36 & $0.708396(7)$ & 0.70456 & $0.512079(7)$ & 0.51175 & -2.7 & 1.2 & $0.282346(6)$ & 0.28233 & -4.8 & 1.3 & 29.84 & 18.14 & 16.27 & 15.58 & 60.93 & 35.97 & n.a. & n.a. \\
\hline Q38 & $0.709780(7)$ & 0.70439 & $0.512076(4)$ & 0.51175 & -2.7 & 1.2 & $0.282339(7)$ & 0.28233 & -5.1 & 1.3 & n.a. & n.a. & n.a. & n.a. & n.a. & n.a. & n.a. & n.a. \\
\hline Q43 & $0.705732(7)$ & 0.70431 & $0.512076(8)$ & 0.51173 & -3.1 & 1.2 & $0.282332(8)$ & 0.28233 & -4.9 & 1.3 & 20.13 & 18.06 & 15.66 & 15.53 & 48.70 & 46.25 & $-4.22(1)$ & $15.59(3)$ \\
\hline \multicolumn{19}{|c|}{ Saglek block mela-aillikites } \\
\hline ST263 & $0.705687(7)$ & 0.70383 & $0.512235(5)$ & 0.51187 & 0.2 & 1.1 & $0.282429(10)$ & 0.28241 & -1.4 & 1.2 & 20.59 & 18.29 & 15.57 & 15.43 & 41.38 & 38.51 & $-5.39(3)$ & $16.04(7)$ \\
\hline ST264 & $0.706639(10)$ & 0.70437 & $0.512069(5)$ & 0.51173 & -3.4 & 1.2 & $0.282369(7)$ & 0.28235 & -4.5 & 1.3 & 19.91 & 18.08 & 15.56 & 15.45 & 43.13 & 39.03 & $-4.82(3)$ & $11.25(3)$ \\
\hline ST266 & $0.707168(7)$ & 0.70365 & $0.512339(3)$ & 0.51199 & 1.9 & 1.0 & $0.282544(8)$ & 0.28253 & 2.0 & 1.0 & 27.66 & 18.43 & 16.06 & 15.51 & 54.17 & 39.28 & $-5.19(2)$ & $11.94(16)$ \\
\hline ST267 & $0.705753(7)$ & 0.70387 & $0.512193(4)$ & 0.51185 & -0.9 & 1.1 & $0.282411(10)$ & 0.28240 & -2.7 & 1.2 & 30.12 & 18.20 & 16.26 & 15.56 & 43.06 & 38.20 & $-3.22(2)$ & $11.68(3)$ \\
\hline
\end{tabular}

Numbers in parentheses are $2 \sigma$-of-the-mean errors for individual isotope ratio measurements.

Duplicate $\mathrm{Hf}$ isotope data for two samples that had additionally been dissolved in teflon autoclaves are listed in the electronic annex A.

Initial isotope ratios calculated for emplacement ages of $582 \mathrm{Ma}$ (Torngat), $606 \mathrm{Ma}$ (Hebron), $568 \mathrm{Ma}$ (Saglek), $578 \mathrm{Ma}$ (Eclipse Harbour), $576 \mathrm{Ma}$ (Killinek Island).

${ }^{\text {b }}$ Initial epsilon Nd values were calculated using ${ }^{147} \mathrm{Sm}$ decay constant of $6.54 \times 10^{-12}$ year ${ }^{-1}$ (Lugmair and Marti, 1978); $\left({ }^{143} \mathrm{Nd} /{ }^{144} \mathrm{Nd}\right)_{\mathrm{CHUR}}=0.512638(\mathrm{Goldstein}$ et al., 1984$) ;\left({ }^{147} \mathrm{Sm} /{ }^{144} \mathrm{Nd}\right)$ CHUR $=0.1967($ Peucat et al., 1988$)$. Initial epsilon $\mathrm{Hf}$ values were calculated using ${ }^{176} \mathrm{Lu}$ decay constant of $1.865 \times 10^{-11}$ eear ${ }^{-1}$ (Scherer et al., 2001); $\left({ }^{176} \mathrm{Hf} /{ }^{177} \mathrm{Hf}\right)_{\mathrm{CHUR}}=0.282843$ and $\left({ }^{176} \mathrm{Lu} /{ }^{177} \mathrm{Hf}\right)_{\mathrm{CHUR}}=0.0342(\mathrm{Patchett}$ et al., 2004$)$.

${ }^{c}$ Depleted Mantle model ages $\left[\mathrm{T}_{(\mathrm{DM})}^{\mathrm{Nd}}\right]$ in Ga were calculated using ${ }^{147} \mathrm{Sm}$ decay constant of $6.54 \times 10^{-12}$ year $^{-1} ;\left({ }^{143} \mathrm{Nd} /{ }^{144} \mathrm{Nd}\right)_{\mathrm{DM}}=0.513150\left(\right.$ Peucat et al., 1988) and $\left({ }^{147} \mathrm{Sm} /{ }^{1{ }^{14}} \mathrm{Nd}\right){ }_{\mathrm{DM}}=0.222(\mathrm{Mich} a r d$ et al., 1985$)$. Depleted Mantle model ages $T_{(\mathrm{DM})}^{\mathrm{Hf}}$ were calculated using ${ }^{176} \mathrm{Lu}$ decay constant of $1.865 \times 10^{-11}$ year ${ }^{-1} ;\left({ }^{176} \mathrm{Hf} /{ }^{177} \mathrm{Hf}\right)_{\mathrm{DM}}=0.283150$ and $\left({ }^{176} \mathrm{Lu} /{ }^{177} \mathrm{Hf}\right)_{\mathrm{DM}}=0.034$ 


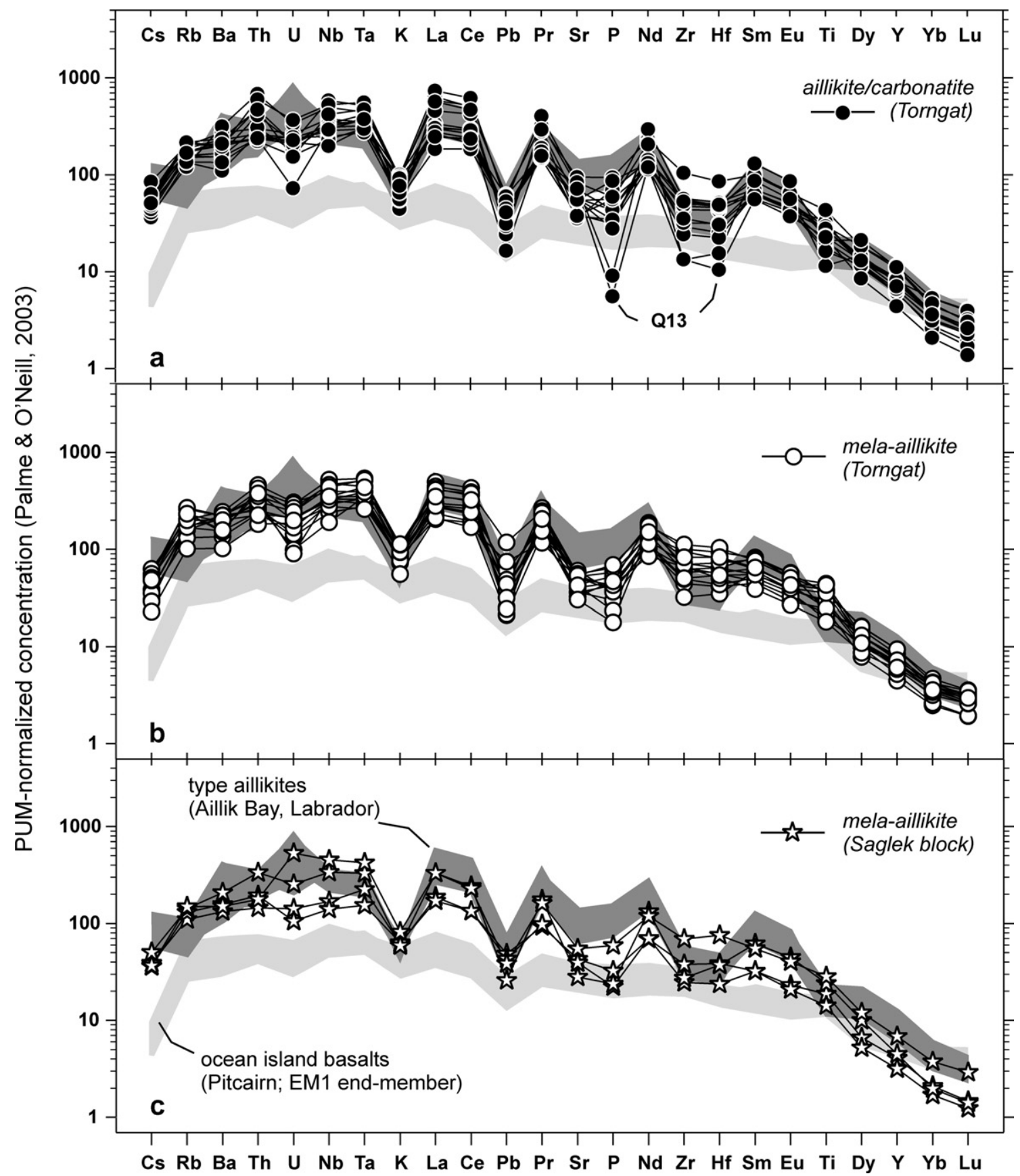

Fig. 5. Primitive upper mantle-normalized (PUM) incompatible element distribution for (a) Torngat aillikites/carbonatites, (b) Torngat melaaillikites, and (c) Saglek block mela-aillikites. Dark grey field shows the incompatible element pattern of type aillikites from Aillik Bay in central Labrador (Tappe et al., 2006, 2007). Pale grey field displays a typical 'Enriched Mantle' derived ocean island basalt trace element pattern for comparison (Eisele et al., 2002). Note the striking similarity between the various aillikite/mela-aillikite patterns from Labrador with only subtle differences, for example, at Cs-Rb and Zr-Hf. PUM values are from Palme and O'Neill (2003).

ST266 from Killinek Island $\left({ }^{87} \mathrm{Sr} /{ }^{86} \mathrm{Sr}_{(\mathrm{i})}=0.70361\right.$; $\left.\varepsilon_{\mathrm{Nd}(\mathrm{i})}=+2.0 ; \quad \varepsilon_{\mathrm{Hf(i)}}=+2.1\right)$ and $\mathrm{ST} 264$ from Saglek $\left({ }^{87} \mathrm{Sr} /{ }^{86} \mathrm{Sr}_{(\mathrm{i})}=0.70437 ; \quad \varepsilon_{\mathrm{Nd}(\mathrm{i})}=-3.4 ; \quad \varepsilon_{\mathrm{Hf}(\mathrm{i})}=-4.5\right)$ represent the isotopically most depleted and enriched coastal Labrador UML, respectively (Figs. 9 and 10).

The initial $\mathrm{Pb}$ isotope compositions of the Torngat aillikites/carbonatites are moderately radiogenic ranging between ${ }^{206} \mathrm{~Pb} /{ }^{204} \mathrm{~Pb}_{(\mathrm{i})}=17.8-18.5,{ }^{207} \mathrm{~Pb} /{ }^{204} \mathrm{~Pb}_{(\mathrm{i})}=15.5-15.7$
(Fig. 11), and ${ }^{208} \mathrm{~Pb} /{ }^{204} \mathrm{~Pb}_{(\mathrm{i})}=37.9-40.5$. Torngat mela-aillikite $\mathrm{Pb}$ isotope compositions $\left({ }^{206} \mathrm{~Pb} /{ }^{204} \mathrm{~Pb}_{(\mathrm{i})}=17.8-18.2\right.$, $\left.{ }^{207} \mathrm{~Pb} /{ }^{204} \mathrm{~Pb}_{(\mathrm{i})}=15.5-15.6,{ }^{208} \mathrm{~Pb} /{ }^{204} \mathrm{~Pb}_{(\mathrm{i})}=36.0-41.9\right)$ are less variable and overlap the unradiogenic end of the Torngat aillikite range. The Saglek block mela-aillikites have ${ }^{206} \mathrm{~Pb} /{ }^{204} \mathrm{~Pb}_{(\mathrm{i})}(18.1-18.3)$ ratios within the range of the Torngat UML, but importantly they tend to have less radiogenic ${ }^{207} \mathrm{~Pb} /{ }^{204} \mathrm{~Pb}_{(\mathrm{i})}(15.4-15.6)$ resembling the urano- 

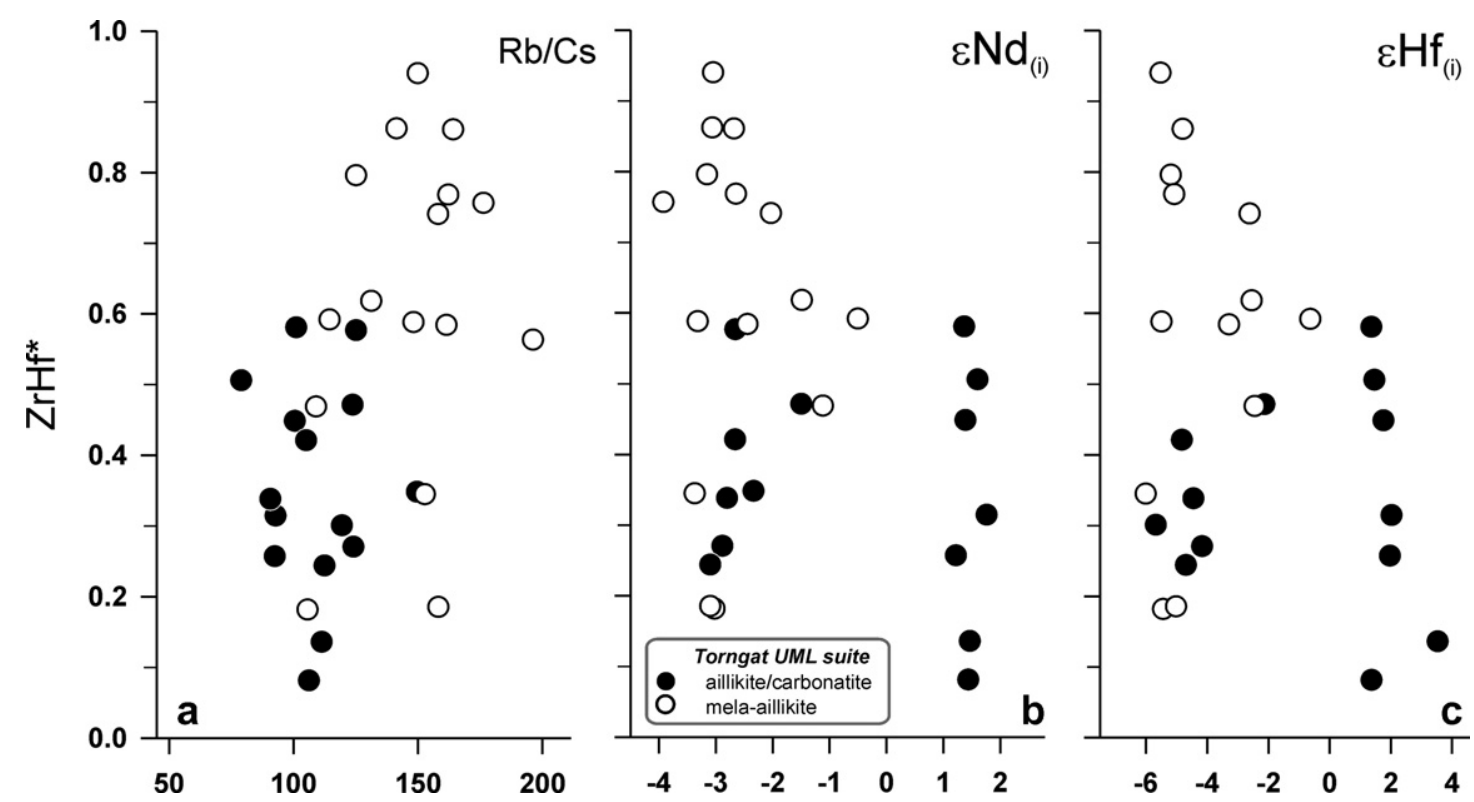

Fig. 6. $\mathrm{ZrHf}^{*}$ vs. (a) $\mathrm{Rb} / \mathrm{Cs}$, (b) initial $\varepsilon \mathrm{Nd}$, and (c) initial $\varepsilon \mathrm{Hf}$ for Torngat aillikite/carbonatite and mela-aillikite dykes from northern Labrador. $\mathrm{ZrHf}^{*}$ is an expression of the negative $\mathrm{Zr}$-Hf anomaly in the PUM-normalized incompatible element pattern in Fig. 5 and defined as PUM-normalized $(\mathrm{Zr}+\mathrm{Hf}) /(\mathrm{Nd}+\mathrm{Sm})$. The Saglek block UMLs are not shown for the sake of clarity.

genic $\mathrm{Pb}$ isotope composition of aillikites/carbonatites from Aillik Bay on the central Labrador coast (Fig. 11).

\subsection{Carbon and oxygen isotope composition}

The carbon and oxygen isotope compositions of the Torngat aillikite carbonates range between -6.8 and $-4.3 \% \quad \delta^{13} \mathrm{C}_{\mathrm{PDB}}$, and $+7.9 \%$ and $+13.8 \% \delta^{18} \mathrm{O}_{\mathrm{SMOW}}$ (Fig. 12; dolomite carbonatite Q13 $=-3.2 \%$ o $\delta^{13} \mathrm{C}_{\mathrm{PDB}}$ ). The comparatively small-volume mela-aillikite groundmass carbonates range between $-6.9 \%$ and $-4.2 \% \delta^{13} \mathrm{C}_{\mathrm{PDB}}$, and $+12.4 \%$ and $+16.1 \% \delta^{18} \mathrm{O}_{\text {SMOW. }}$. Groundmass carbonates of the Saglek $\left(-4.8 \% \delta^{13} \mathrm{C}_{\mathrm{PDB}} ; 11.2 \% \delta^{18} \mathrm{O}_{\text {SMOw }}\right)$, Killinek Island $\left(-5.2 \% \delta^{13} \mathrm{C}_{\mathrm{PDB}} ; 11.9 \% \delta^{18} \mathrm{O}_{\mathrm{SMOW}}\right)$, and Hebron $\left(-5.4 \% \delta^{13} \mathrm{C}_{\mathrm{PDB}} ; 16.0 \% \delta^{18} \mathrm{O}_{\text {SMOW }}\right)$ mela-aillikites fall within the Torngat UML compositional range. Eclipse Harbour mela-aillikite carbonate $\left(-3.2 \%\right.$ o $\delta^{13} \mathrm{C}_{\mathrm{PDB}} ; 11.7 \%$ $\left.\delta^{18} \mathrm{O}_{\text {SMOW }}\right)$ compositionally resembles the Torngat dolomite carbonatite Q13; these carbonates contain isotopically heavier carbon than their Torngat UML analogues (Fig. 12). The isotopically most primary 'mantle-like' Torngat aillikite carbonates overlap the carbon and oxygen isotope composition of carbonates from type aillikites at Aillik Bay in central Labrador (Tappe et al., 2006).

\section{DISCUSSION}

Aillikite/carbonatite and mela-aillikite magma production occurred beneath the Torngat Mountains and the Saglek block along the western NAC margin between ca. 610 to 565 Ma (Figs. 1, 4). This UML magmatism was contemporaneous with long lasting aillikite and carbonatite igneous activity in the Aillik Bay area, central Labrador (ca. 590 to $555 \mathrm{Ma}$ ), and the Sisimiut-Sarfartoq-Maniitsoq areas of western Greenland (ca. 610 to $550 \mathrm{Ma}$ ) at the southern and northern NAC margins, respectively. Some of the Torngat and West Greenland UML/carbonatite dykes have been confirmed to contain diamonds (Digonnet et al., 2000; Nielsen and Jensen, 2005; Tappe et al., 2005). Furthermore, Bizzarro and Stevenson (2003) demonstrated that garnet peridotite xenoliths recovered from the West Greenland examples had last equilibrated at pressures of up to $6.3 \mathrm{GPa}$. Thus, UML and carbonatite magma production beneath the NAC occurred, at least in some areas, at depths greater than $150 \mathrm{~km}$, i.e., well within the diamond stability field.

In the following discussion we place constraints on the heterogeneities within the deep source region of the Torngat UML suite as revealed by their wide range of $\mathrm{Sr}-\mathrm{Nd}-\mathrm{Hf}-$ $\mathrm{Pb}$ isotope compositions. An attempt is made to assign the contrasting isotopic components to discernable mineral assemblages and to shed light onto the melting mechanisms by which these components/assemblages were mobilized producing the aillikite/carbonatite and mela-aillikite continuum.

\subsection{Source mineralogical control on aillikite/carbonatite and mela-aillikite magma compositions}

\subsubsection{High $\mathrm{Mg}$ and compatible trace elements}

Despite the distinctly different carbonate modes and $\mathrm{SiO}_{2}, \mathrm{Al}_{2} \mathrm{O}_{3}, \mathrm{Na}_{2} \mathrm{O}$ contents of the Torngat aillikites/carbonatites and mela-aillikites, their high $\mathrm{MgO}, \mathrm{Ni}$, and $\mathrm{Cr}$ contents overlap and meet the criteria for near-primary peridotitic mantle-derived magmas (e.g., $\mathrm{Mg} \#>68$; $\mathrm{Ni}>320$ ppm; cf. Frey et al., 1978). Furthermore, the Torngat aillikites, as well as many other carbonate-rich UML from Labrador and West Greenland, broadly resemble near-solidus melt compositions experimentally produced from synthetic carbonate-bearing peridotite in the 


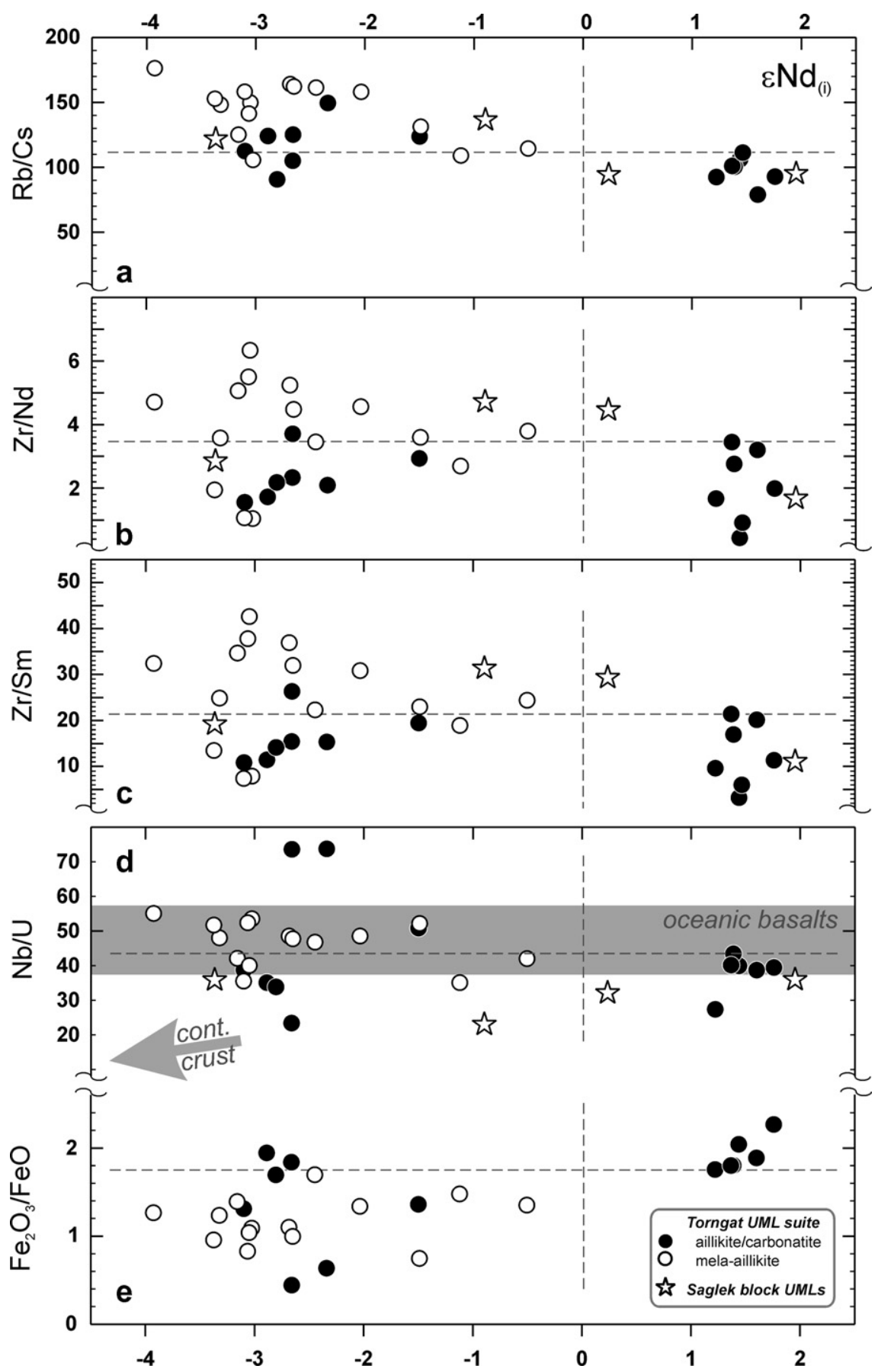

Fig. 7. Incompatible element ratios (a-d) and $\mathrm{Fe}_{2} \mathrm{O}_{3} / \mathrm{FeO}$ ratios (e) vs. initial $\varepsilon \mathrm{Nd}$ of the Torngat and Saglek block aillikite/carbonatite and mela-aillikite dykes. The grey band shows the range of $\mathrm{Nb} / \mathrm{U}$ ratios for oceanic basalts (Hofmann et al., 1986). The arrow points towards the compositions of typical continental crust lithologies from cratonic areas. The dashed lines are visual aids enabling a better recognition of the subtle trace element differences between the isotopically depleted (positive $\varepsilon \mathrm{Nd}$ ) and enriched samples (negative $\varepsilon \mathrm{Nd}$ ). See text for further explanations.

CMAS- $-\mathrm{CO}_{2}$ system at $6 \mathrm{GPa}$ (Dalton and Presnall, 1998) (Fig. 3). Although these experiments have limitations as to their applicability to natural magmas, because important components such as alkalis, $\mathrm{H}_{2} \mathrm{O}, \mathrm{FeO}, \mathrm{TiO}_{2}$, and $\mathrm{P}_{2} \mathrm{O}_{5}$ are lacking, they demonstrate that partial melts produced from garnet peridotite under $\mathrm{CO}_{2}$-rich high-pressure conditions can have intermediate compositions between carbonate and ultramafic silicate melts. It is important here to note that the primitive $\mathrm{CO}_{2}$-poor Torngat mela-aillikites are different in some respects from these CMAS- $\mathrm{CO}_{2}$ experimental melt compositions; they have higher $\mathrm{Al}_{2} \mathrm{O}_{3}$ and lower $\mathrm{MgO}$ and $\mathrm{CaO}$ than the high-pressure run products at a given $\mathrm{SiO}_{2}$ content (Fig. 3). However, mela-aillikite major element compositions (e.g., $\mathrm{SiO}_{2}, \mathrm{TiO}_{2}, \mathrm{Al}_{2} \mathrm{O}_{3}, \mathrm{CaO}$, and 


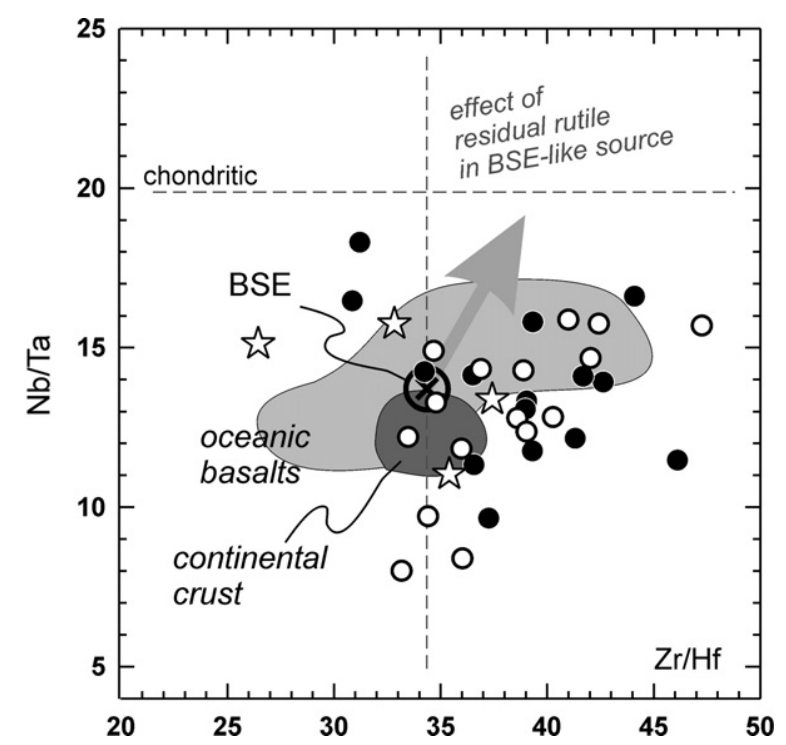

Fig. 8. $\mathrm{Nb} / \mathrm{Ta}$ vs. $\mathrm{Zr} / \mathrm{Hf}$ ratios for Torngat and Saglek block aillikite/carbonatite and mela-aillikite dykes from northern Labrador. Despite significant variability in these HFSE ratios, no differences are apparent among the UML types. Fields for oceanic basalts and continental crust materials are shown for comparison (Pfänder et al., 2007). The arrow indicates a presumed trend of a melt formed in the presence of residual rutile with Bulk Silicate Earth-like (BSE) $\mathrm{Nb} / \mathrm{Ta}$ and $\mathrm{Zr} / \mathrm{Hf}$.

alkalis) are similar to melts produced experimentally from a synthetic Ti-rich hydrous ultrabasic glass at 4 and $8 \mathrm{GPa}$ (Konzett, 1997) (Fig. 3). This concordance strongly implies enhanced involvement of trace mineral assemblages ('veins') within peridotite during magma generation, as also suggested by a reconnaissance high-pressure melting experiment on a melilite-bearing UML from Antarctica (Foley and Andronikov, 2003).

The high $\mathrm{MgO}$ of the Torngat UMLs is coupled to extremely high incompatible element concentrations (i.e., up to $700 \times$ PUM for the LREE), which are unlikely to be attained by partial melting of peridotite alone, not even at reasonably low degrees of partial melting within an incipient melting regime (Green and Falloon, 1998). This apparent conflict can be readily explained by the presence of mixed source rocks (Irving, 1980; Menzies, 1983; Foley, 1992a,b; Harte et al., 1993), where the vast majority of incompatible elements reside in non-peridotitic trace minerals such as phlogopite, Ti-oxides, and apatite (among others) that have been reported from composite mantle xenoliths. Hence, the Rb-Sr, Sm-Nd, Lu-Hf, and U-Th$\mathrm{Pb}$ radiogenic isotope systems utilized here are unlikely to fingerprint signatures from a pure peridotite substrate, for which Os isotope compositions would be more suitable (Carlson et al., 1996, 2007). Rather, they provide time-integrated information about the metasomatic component(s) in the mantle source region.

The large spread in Torngat aillikite/carbonatite and mela-aillikite $\mathrm{Sr}-\mathrm{Nd}-\mathrm{Hf}-\mathrm{Pb}$ isotope ratios (Figs. 9-11), with marked correlations between isotope systems, clearly reflects that more than one mantle source enrichment event had occurred during the protracted geologic history of the NAC. However, the strong similarity of the incompatible element distribution among UML types (Fig. 5) requires that multiple metasomatic overprinting of the peridotite substrate had produced some common trace element-rich minerals, which controlled incompatible element partitioning during both aillikite and mela-aillikite melt production. In order to better understand the metasomatic history of the Torngat UML magma source region, we now explore the nature of potential metasomatic minerals by integration of petrologic, geochemical, and isotopic information retrieved from the observed magma compositions.

\subsubsection{High $K$ and LFSE}

Aillikite/carbonatite and mela-aillikite magma clearly segregated from a mantle source region that contained an early melting hydrous K-bearing phase. Phlogopite and K-richterite are known from metasomatized mantle assemblages (Dawson and Smith, 1977; Waters, 1987; Ionov and Hofmann, 1995; Grégoire et al., 2002, 2003), both being stable to pressures above $7 \mathrm{GPa}$ (Sudo and Tatsumi, 1990; Foley, 1991; Konzett et al., 1997; Konzett and Ulmer, 1999). Phlogopite is considered the most likely K-bearing phase in the melting assemblage of aillikites because of its potential to produce silica-undersaturated melts with extremely high $\mathrm{K}_{2} \mathrm{O} / \mathrm{Na}_{2} \mathrm{O}$ ratios ( $11 \pm 5$ for Torngat aillikites). In contrast, K-richterite was demonstrated to melt out close to the solidus of ultramafic assemblages, yielding $\mathrm{SiO}_{2}$-richer melt compositions that are more akin to lamproites (Konzett, 1997; Foley et al., 1999). The Torngat mela-aillikites show exactly this increase in $\mathrm{SiO}_{2}$ (Fig. 3), as well as lowered $\mathrm{K}_{2} \mathrm{O} / \mathrm{Na}_{2} \mathrm{O}(6 \pm 3)$, that would be expected if $\mathrm{K}$ richterite enters a carbonate-rich melt similar to aillikite. However, $\mathrm{K}_{2} \mathrm{O}$ of the mela-aillikites is near-constant $(2.7 \pm 0.5 \mathrm{wt} \%)$ and overlaps the aillikites $(2.4 \pm 0.5 \mathrm{wt} \%)$, which indicates buffering by residual phlogopite during melt segregation. We reach this conclusion because $\mathrm{K}$ is a stoichiometric component in phlogopite and the $\mathrm{K}_{2} \mathrm{O}$ content in the melt will be near-constant as long as this phase is residual and the melting mode remains constant. Accordingly, $\mathrm{K}$ varies mainly as a function of the proportion to which phlogopite enters the melt. The $\mathrm{K}_{2} \mathrm{O}$ content of a melt in equilibrium with mantle phlogopite which melts to an extent of $\sim 20-50 \%$ (Greenough, 1988) is $\sim 1.6-5 \mathrm{wt} \%$. The average $\mathrm{K}_{2} \mathrm{O}$ contents of aillikite and mela-aillikite are at the lower end of this range, which is consistent with lower $\mathrm{K}$-saturation levels in $\mathrm{SiO}_{2}$-poor melts produced under $\mathrm{CO}_{2}$-rich conditions (Mengel and Green, 1989; Rogers et al., 1992). Note that phlogopite precipitation from aillikite and mela-aillikite magma en route to the surface can be ruled out as the principle cause for the relatively low $\mathrm{K}_{2} \mathrm{O}$ contents, because of their high $\mathrm{MgO}$ contents and the presence of mantle-derived materials such as diamond, which is indicative of rapid magma ascent.

The impact of residual phlogopite on the incompatible element patterns of aillikites and mela-aillikites is clearly seen at the relative depletions of $\mathrm{Cs}$ and $\mathrm{Rb}$, and the pronounced trough at $\mathrm{K}$ and $\mathrm{Pb}$ if compared to the highly incompatible HFSE (Th, U, Nb, Ta) and LREE (Fig. 5). The higher $\mathrm{Rb} / \mathrm{Cs}$ of the majority of mela-aillikites com- 


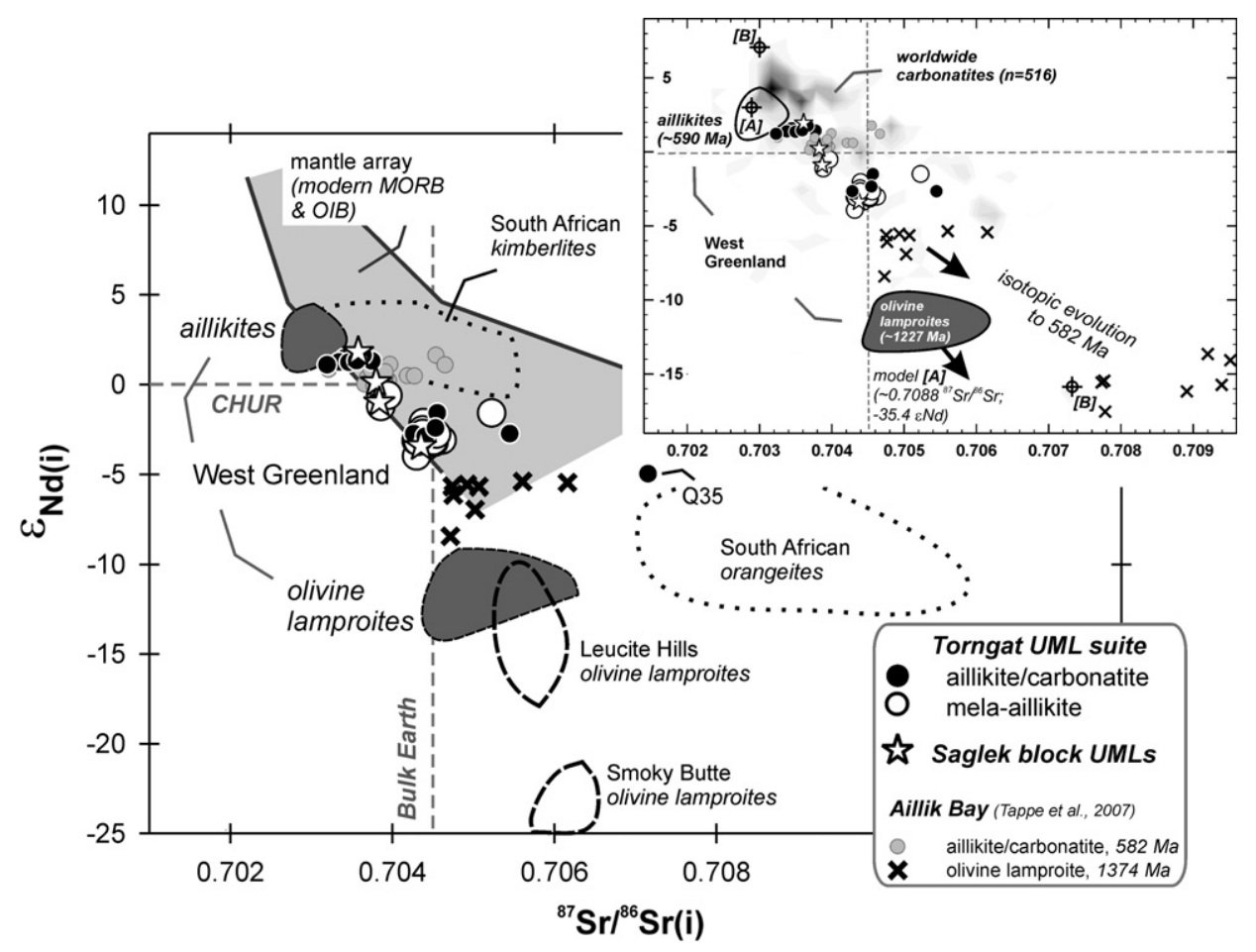

Fig. 9. Initial $\varepsilon N d$ vs. ${ }^{87} \mathrm{Sr} /{ }^{86} \mathrm{Sr}$ for Torngat and Saglek block aillikite/carbonatite and mela-aillikite dykes from northern Labrador. The inset shows the new Torngat isotope data in relationship to our choice of end-member compositions utilized for binary mixing calculations as illustrated in greater detail in Fig. 14 and Table 4. Model $\boldsymbol{A}$ interprets the Torngat aillikite/carbonatite and mela-aillikite compositional continuum as binary mixtures between melts derived from long-term mildly depleted (worldwide carbonatites) and strongly enriched (West Greenland olivine lamproite) isotope end-member components. A similar approach was adopted in model B, where the long-term depleted end-member is represented by the most depleted carbonatite that has yet been reported (Kola Alkaline Province) and the enriched endmember by central Labrador Aillik Bay olivine lamproite derived from ancient hydrous metasomatized SCLM. Fields for West Greenland aillikites and olivine lamproites (Nelson, 1989), Leucite Hills and Smoky Butte olivine lamproites (Fraser et al., 1985), and South African kimberlites and orangeites (Nowell et al., 2004) are shown for comparison. Data for the aillikite/carbonatites and olivine lamproites from Aillik Bay can be found in Tappe et al. (2007).

pared to aillikites at a similar $\mathrm{K}$ concentration level (Fig. 6a) could have been produced by the inferred K-richterite fusion, because ${ }^{\mathrm{Krcht} / \mathrm{melt}^{2}} \mathrm{D}_{\mathrm{Rb}}$ is almost two orders of magnitude lower than ${ }^{\mathrm{ph} l / m e l t} \mathrm{D}_{\mathrm{Rb}}$ (Foley et al., 1996; Tiepolo et al., 2003). However, $\mathrm{Cs}$ and $\mathrm{Rb}$ concentrations of this phase are an order of magnitude lower than in mantle phlogopite (Grégoire et al., 2002), which is an essential phase during both aillikite and mela-aillikite magma production. Moreover, K-richterite is almost certainly consumed during partial melting (Konzett, 1997; Foley et al., 1999), and so does not buffer the incompatible element distribution. Thus, the difference in $\mathrm{Rb} / \mathrm{Cs}$ may indicate the presence of distinct phlogopite populations with variable $\mathrm{Rb}$ and $\mathrm{Cs}$ concentrations. Such differences in the trace element content among distinct phlogopite populations may be the result of growth from contrasting metasomatic agents (i.e., hydrous- vs. carbonate-dominated) during multiple metasomatic events, which are known to have overprinted the cratonic mantle in the region (Griffin et al., 2004; Tappe et al., 2007). This interpretation is consistent with a slightly negative correlation between $\mathrm{Rb} / \mathrm{Cs}$ and ${ }_{\varepsilon} \mathrm{Nd}$ (Fig. 7a) implying the high $\mathrm{Rb} / \mathrm{Cs}$ signature of melaaillikites, and some aillikites, fingerprints an old hydrous metasomatized mantle lithology (phlogopite- and K-richte- rite-bearing) as opposed to a younger phlogopite-bearing metasomatic assemblage that largely contributed to the aillikite/carbonatite magma.

\subsubsection{High Ti and HFSE}

The extremely high $\mathrm{TiO}_{2}$ concentrations of aillikites $(5.3 \pm 1.6 \mathrm{wt} \%)$ and mela-aillikites $(6.6 \pm 1.9 \mathrm{wt} \%)$ cannot be explained solely by melting Ti-rich silicates such as phlogopite (cf. Konzett, 1997). As this high $\mathrm{TiO}_{2}$ is coupled to extremely high $\mathrm{Nb}-\mathrm{Ta}$ abundances $(213 \pm 68$ and $212 \pm 58 \mathrm{ppm} \mathrm{Nb}$, respectively; >200 $\times$ PUM), an early melting Ti-rich oxide phase seems to have largely contributed to the HFSE budget during melt production. Melting experiments on non-peridotitic ultramafic assemblages showed that ilmenite melts out quickly, whereas rutile persists to higher temperatures (Foley et al., 1999). This observation makes ilmenite the more likely Ti-phase that contributed to both aillikite and mela-aillikite magmas accounting for the similar high levels of $\mathrm{Ti}, \mathrm{Nb}-\mathrm{Ta}$, and $\mathrm{Zr}-\mathrm{Hf}$ in these rock types (Fig. 5). Furthermore, both the $\mathrm{Nb}-\mathrm{Ta}$ and the $\mathrm{Zr}-\mathrm{Hf}$ pair are mildly compatible with ilmenite/melt partition coefficients close to unity (Klemme et al., 2006), which is consistent with the absence of significant $\mathrm{Nb} / \mathrm{Ta}$ and $\mathrm{Zr} / \mathrm{Hf}$ fractionation in the aillikite and 


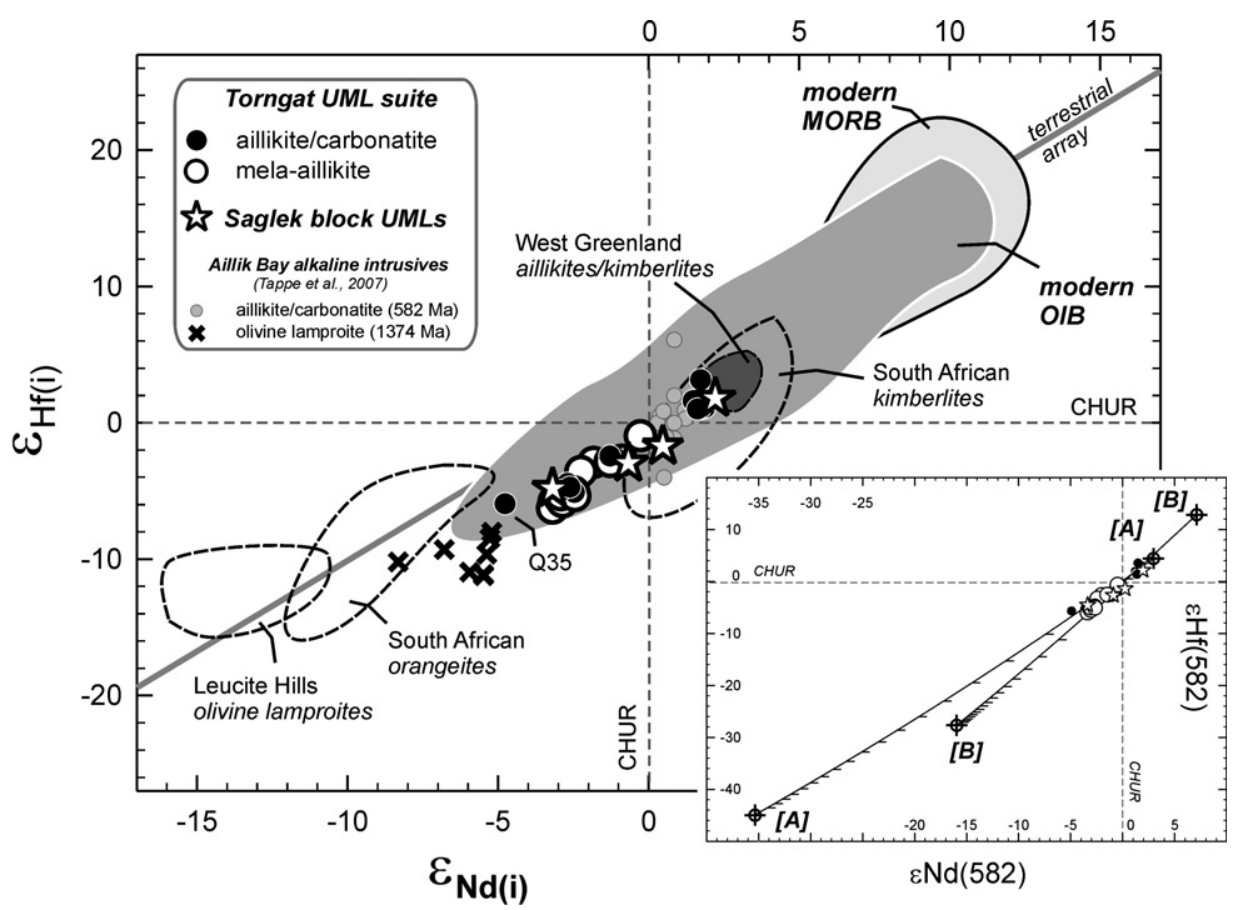

Fig. 10. Initial $\varepsilon H f$ vs. $\varepsilon N d$ for Torngat and Saglek block aillikite/carbonatite and mela-aillikite dykes from northern Labrador. The inset shows two mixing hyperbolae (model $\boldsymbol{A}$ and $\boldsymbol{B}$; see Figs. 9, 14, and Table 4) constructed between isotopically depleted radiogenic components (proxy for convective mantle-derived carbonate melt) and isotopically enriched unradiogenic components (proxy for hydrous metasomatized cratonic mantle-derived potassic melt). The underlying modelling assumptions are described in the main text and in the caption to Fig. 14. Tick marks are at 5\% intervals. Fields for West Greenland aillikites/kimberlites (Gaffney et al., 2007), South African kimberlites and orangeites (Nowell et al., 2004), Leucite Hills olivine lamproites (Salters and Hart, 1991), and modern oceanic basalts (compilation retrieved from http://georoc.mpch-mainz.gwdg.de/georoc/) are shown for comparison. Data for the aillikite/carbonatites and olivine lamproites from Aillik Bay can be found in Tappe et al. (2007). Terrestrial array after Vervoort et al. (1999).

mela-aillikite magmas (Fig. 8). Some mela-aillikites show a marked increase in $\mathrm{Ti}$ and $\mathrm{Zr}$-Hf (Fig. 5) accompanied by slightly increasing $\mathrm{Nb}-\mathrm{Ta}$ contents. This appears to indicate that more ilmenite melted locally, presumably reflecting its heterogeneous distribution within the metasomatic source assemblages. Note that rutile is unlikely to be the source of this additional HFSE contribution to some of the mela-aillikites, because it would have significantly increased the $\mathrm{Nb}-\mathrm{Ta}$ content and $\mathrm{Nb} / \mathrm{Ta}$ ratio of the melt (Foley et al., 2000; Klemme et al., 2005), which is not observed (Fig. 8). By analogy to the elevated Rb/Cs of mela-aillikites, $\mathrm{Zr} / \mathrm{Nd}$ and $\mathrm{Zr} / \mathrm{Sm}$ ratios and thus the $\mathrm{ZrHf}^{*}$ value of mela-aillikites (Fig. $6 \mathrm{~b}$ and c) show a rough negative correlation with $\varepsilon \mathrm{Hf}$ and $\varepsilon \mathrm{Nd}$ (Fig. $7 \mathrm{~b}$ and $\mathrm{c}$ ). This implies that the additionally fused $\mathrm{HFSE}^{4+}$ resided in a Ti-oxide phase, most likely ilmenite, which appears to be part of the old hydrous metasomatic assemblage. It furthermore appears that some of the carbonate-rich aillikites with negative $\varepsilon \mathrm{Nd}$ and ${ }_{\varepsilon} \mathrm{Hf}$ isotope signatures have assimilated some of this longterm enriched material in a rather erratic fashion given their still low $\mathrm{Rb} / \mathrm{Cs}, \mathrm{Zr} / \mathrm{Nd}$, and $\mathrm{Zr} / \mathrm{Sm}$ ratios (Fig. $7 \mathrm{a}-\mathrm{c}$ ), as well as low $\mathrm{SiO}_{2}$ contents (Fig. 3).

\subsubsection{High P, LREE, and Th/U}

Apatite must be an essential constituent of the source region of aillikites and mela-aillikites given their similarly high buffered $\mathrm{P}_{2} \mathrm{O}_{5}$ concentrations $(1.1 \pm 0.7$ and
$0.8 \pm 0.3 \mathrm{wt} \%$ ). It is among the most important phases in controlling the REE and Th- $\mathrm{U}$ budgets of alkaline mantle-derived melts (O'Reilly and Griffin, 2000; Klemme and Dalpe, 2003), and the imprint of residual apatite on the incompatible element patterns of the Torngat UMLs may be seen at the $\mathrm{Sr}-\mathrm{P}$ trough (Fig. 5). Interestingly, this trough also occurs in the type aillikite pattern from Aillik Bay, but less pronounced. The Torngat UMLs have higher $\mathrm{Th} / \mathrm{U}$ ratios (U trough) than the Aillik Bay type aillikites (U spike), which have higher modal carbonate contents but otherwise share an identical mineral assemblage (Tappe et al., 2006). Given the strong influence of melt composition (i.e., silica activity) on apatite/melt trace element partitioning (Watson and Green, 1981; Klemme and Dalpe, 2003), the observed difference in $\mathrm{Th} / \mathrm{U}$ between the UML occurrences may reflect the presence of distinctively different apatite within the metasomatic source assemblages, i.e., apatite populations precipitated from silica-rich hydrous and/or carbonate-rich melts/fluids.

\subsection{Origin of the carbonate}

The $\delta^{13} \mathrm{C}$ values of the aillikite and mela-aillikite carbonate fractions overlap (Fig. 12), with values consistent for primary mantle-derived carbon (Deines, 2002). However, only few aillikite carbonates exhibit mantle-like oxygen isotope compositions, i.e. $<9 \%{ }^{18} \mathrm{O}_{\text {SMOW }}$ (Clarke et al., 1994; 


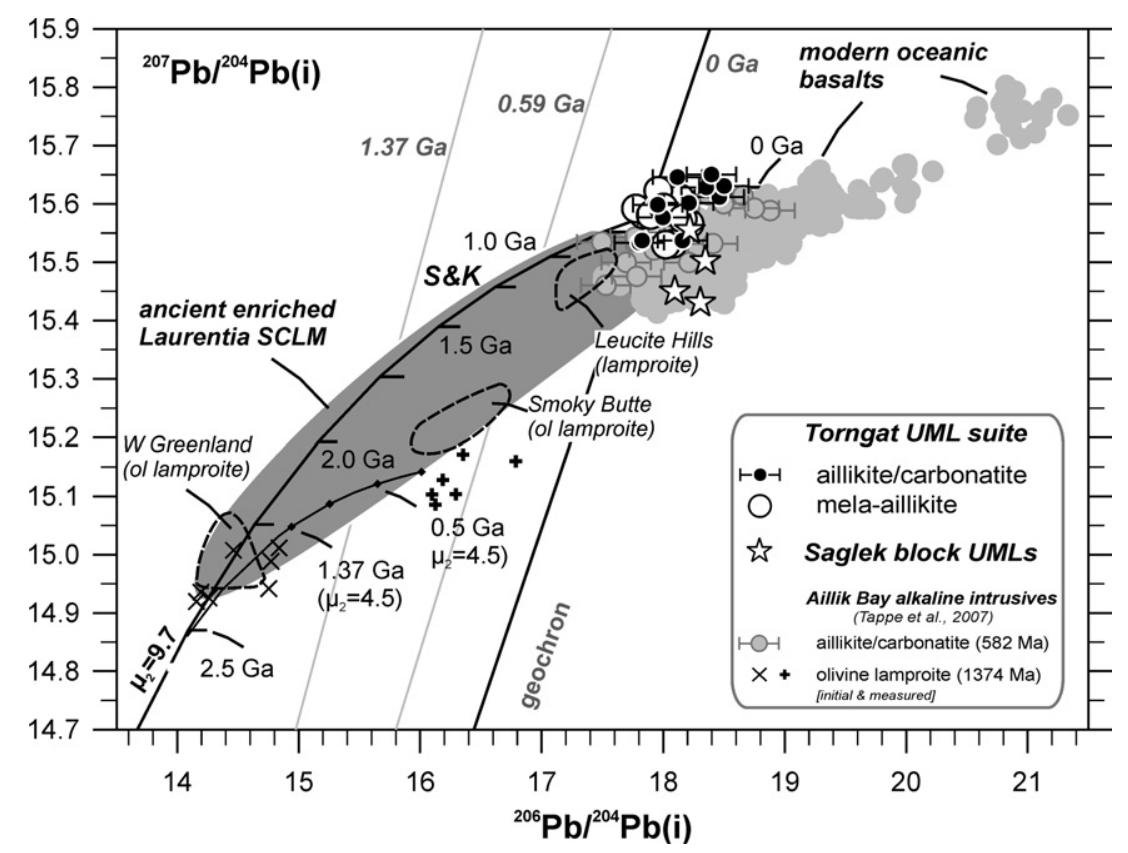

Fig. 11. Initial ${ }^{207} \mathrm{~Pb} /{ }^{204} \mathrm{~Pb}$ vs. ${ }^{206} \mathrm{~Pb} /{ }^{204} \mathrm{~Pb}$ for Torngat and Saglek block aillikite/carbonatite and mela-aillikite dykes from northern Labrador. The cumulative $2 \sigma$ uncertainties for the initialized lead isotope ratios are relatively large as indicated by the whiskers for aillikites due to the combined effects of uncertainties from the high measured $\mathrm{U} / \mathrm{Pb}$ ratios and large age corrections. The Torngat UMLs fall at the end of the Stacey and Kramers (1975) terrestrial Pb evolution curve (tick marks for 250-Myr intervals) close to the present-day geochron. Note the less radiogenic ${ }^{207} \mathrm{~Pb} /{ }^{204} \mathrm{~Pb}$ of the Saglek block UMLs compared to their Torngat analogues at similar ${ }^{206} \mathrm{~Pb} /{ }^{204} \mathrm{~Pb}$. The Mesoproterozoic olivine lamproites from Aillik Bay in central Labrador and from West Greenland have been interpreted as being derived from a metasomatic SCLM component that was withdrawn from the convective mantle at ca. $2.3 \mathrm{Ga}$ and subsequently evolved with a low second-stage $\mu_{2}(\sim 4.5$; Tappe et al., 2007). Such an unradiogenic $\mathrm{Pb}$ within ancient metasomatized cratonic SCLM may have imparted $\operatorname{low}^{206} \mathrm{~Pb} /{ }^{204} \mathrm{~Pb}$ and ${ }^{207} \mathrm{~Pb} /{ }^{204} \mathrm{~Pb}$ to the carbonate-rich Torngat aillikites thereby shifting $\mathrm{Pb}$ isotope compositions towards mela-aillikites. Ancient enriched Laurentia SCLM as defined by potassic anorogenic magma compositions and micaceous SCLM xenoliths: Fraser et al. (1985), Dudas et al. (1987), Nelson (1989), Carlson and Irving (1994), Peterson et al. (1994), O’Brien et al. (1995), Mirnejad and Bell (2006), and Tappe et al. (2007). Modern oceanic basalt data were compiled from literature and database sources (http://georoc.mpch-mainz.gwdg.de/georoc/). The position of the present-day geochron was calculated for $4.55 \mathrm{Ga}$ and initial $\mathrm{Pb}$ isotope composition similar to the Canyon Diablo troilite.

Keller and Hoefs, 1995). The majority of aillikite and all mela-aillikite carbonates have $\delta^{18} \mathrm{O}_{\text {SMOw }}$ values $>9 \%$, i.e., not in equilibrium with mantle peridotite $(5.5 \pm 0.5 \%$; Mattey et al., 1994). Mela-aillikite carbonates tend to be heavier in oxygen, typically $>12 \%{ }^{18} \mathrm{O}_{\mathrm{SMO}}$, compared to aillikite carbonates (Fig. 12).

Large variations in $\delta^{18} \mathrm{O}$ accompanied by only small variations in $\delta^{13} \mathrm{C}$ are known from carbonates in hydrothermally altered systems and volatile-rich ultramafic intrusives such as kimberlites (e.g., Kirkley et al., 1989; Santos and Clayton, 1995; Wilson et al., 2007). The Torngat UML formed rapidly cooled small intrusive dykes that appear pristine down to the microscopic scale with olivine being preserved in most instances (Fig. 2). Furthermore, concentrations of fluid-mobile elements such as $\mathrm{Cs}$ and Ba show only a restricted range (Fig. 5) and do not correlate with $\delta^{18} \mathrm{O}$. This excludes the possibility of carbonate oxygen isotope overprinting by externally-derived meteoric fluids. Wilson et al. (2007) modelled the closed-system oxygen isotope fractionation between primary kimberlite magmatic carbonates and coexisting magmatic $\mathrm{CO}_{2}-\mathrm{H}_{2} \mathrm{O}$ fluids as a function of temperature, fluid $\mathrm{CO}_{2} / \mathrm{H}_{2} \mathrm{O}$ ratio, and fluid/solid ratio. They demonstrated the strong influence of the fluid $\mathrm{CO}_{2} / \mathrm{H}_{2} \mathrm{O}$ ratio on the magnitude of oxygen isotopic fractionation at temperatures below $750{ }^{\circ} \mathrm{C}$, i.e., along a magmatic cooling path (see temperature scales in Fig. 12). For example, the $\delta^{18} \mathrm{O}$ value of primary magmatic carbonate increases from approximately $7.5 \%$ to $17 \%$ upon cooling $\left(1100\right.$ to $\left.100{ }^{\circ} \mathrm{C}\right)$ in the presence of a $\mathrm{H}_{2} \mathrm{O}$-rich equilibrium magmatic fluid (low molar $\mathrm{CO}_{2} / \mathrm{H}_{2} \mathrm{O}$ of 0.25 ; Fig. 12). In the presence of a $\mathrm{CO}_{2}$-richer magmatic fluid $\left(\mathrm{CO}_{2} / \mathrm{H}_{2} \mathrm{O}\right.$ of 0.5$)$, however, the isotopic shift is lower $\left(7.5-13 \% \delta^{18} \mathrm{O}\right)$ over the same temperature range (Wilson et al., 2007). These modelled values compare quite well with the observed range of $\delta^{18} \mathrm{O}$ values for the Torngat UML suite, i.e., the $\mathrm{CO}_{2}$-rich aillikites (bulk-rock $\mathrm{CO}_{2} / \mathrm{H}_{2} \mathrm{O}$ ratios of up to 5) show generally lower $\delta^{18} \mathrm{O}$ values than the $\mathrm{H}_{2} \mathrm{O}$ rich mela-aillikites $\left(\mathrm{CO}_{2} / \mathrm{H}_{2} \mathrm{O}\right.$ of $0.4 \pm 0.4$; Fig. 12).

Hence, it appears that aillikite and mela-aillikite magmas tapped the same carbonated mantle source region given their 'primitive' overlapping carbonate $\delta^{13} \mathrm{C}$ values. The observed variation in the carbonate oxygen isotope data is consistent with sub-solidus (low-temperature) isotope fractionation between this mantle-derived carbonate and magmatic fluids with distinctively different $\mathrm{CO}_{2} / \mathrm{H}_{2} \mathrm{O}$. Our earlier proposal that explained the co-occurrence of carbonate-rich and carbonate-poor UML magma types in the Torngat Mountains by near-surface $\mathrm{CO}_{2}$-degassing 


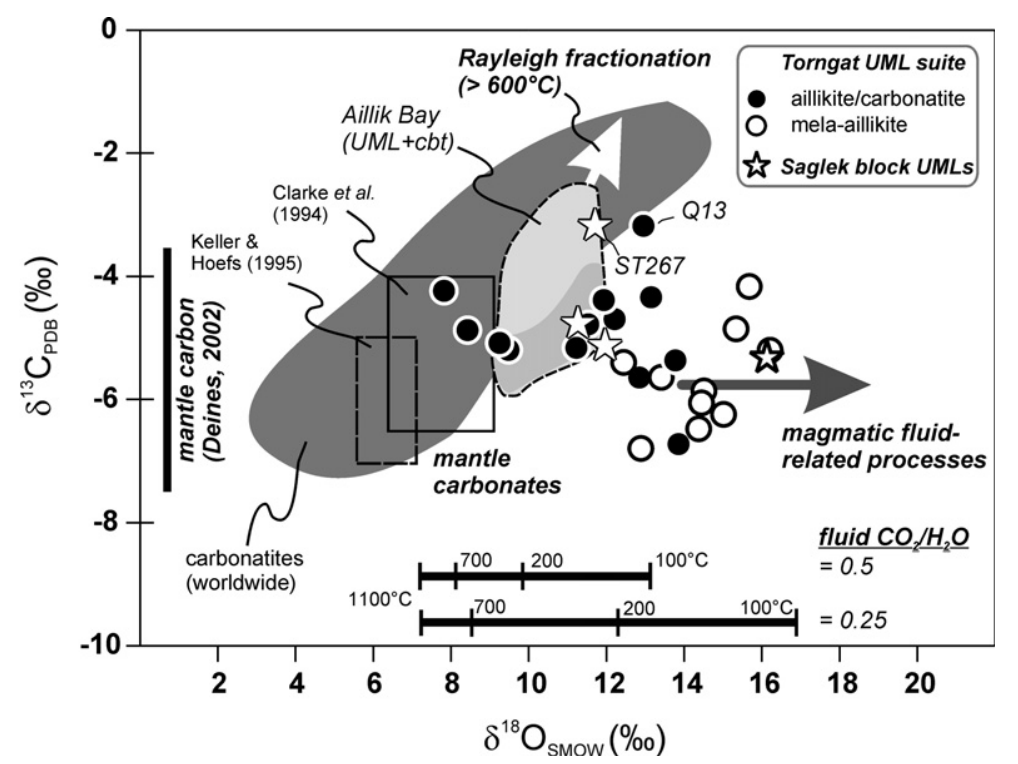

Fig. 12. Carbon and oxygen isotope composition (expressed as $\% \delta^{13} \mathrm{C}$ and $\delta^{18} \mathrm{O}$ relative to PDB and SMOW, respectively) of bulk-rock carbonate fractions from the Torngat and Saglek block aillikite/carbonatite and mela-aillikite dykes from northern Labrador. Open-system $\mathrm{CO}_{2}$-degassing and concomittant Rayleigh fractionation processes, as well as low-temperature magmatic fluid-related processes have the potential to change primary stable isotope compositions as illustrated by arrows (see Deines, 2002, 2004; Wilson et al., 2007). The temperature scales at the bottom refer to two closed-system isotopic fractionation models between primary magmatic carbonates and magmatic fluids with different $\mathrm{CO}_{2} / \mathrm{H}_{2} \mathrm{O}$. See main text and Wilson et al. (2007) for more details. Dark grey field for worldwide carbonatites was compiled from literature sources and data are available from the first author upon request. An outline for the Aillik Bay aillikite (darker) and carbonatite (paler) bulk-rock carbonates is shown for comparison (Tappe et al., 2006). Symbol size is larger than the $2 \sigma$ uncertainty.

(Tappe et al., 2004) appears invalid in view of the new isotope data. Such an open-system Rayleigh fractionation process would have produced a significant difference in the carbon isotope composition (Chacko et al., 1991; Ray and Ramesh, 2000; Deines, 2004), which is not observed. Hence this implies that the different bulk-rock $\mathrm{CO}_{2} / \mathrm{H}_{2} \mathrm{O}$ ratios and modal carbonate abundances of aillikites and mela-aillikites are inherited from the magma source region. The stable isotope evidence for a primordial origin of the carbon for the $\mathrm{CO}_{2}$-rich magmatism of the NAC between 610 and $550 \mathrm{Ma}$ (see also Tappe et al., 2006) suggests the recently inferred 'surface' origin of this magmatic carbonate within the 'subducted oceanic crust model' by Gaffney et al. (2007) is incorrect.

\subsection{Origins of the depleted and enriched $\mathrm{Sr}-\mathrm{Nd}-\mathrm{Hf}$ isotope components}

Torngat aillikites/carbonatites and mela-aillikites form well defined arrays in $\mathrm{Sr}-\mathrm{Nd}-\mathrm{Hf}$ isotope space (Figs. 9 and 10) implying that the peridotite mantle source region contained two isotopically distinct metasomatic assemblages. An isotopically mildly depleted isotope component is identified in some aillikites/carbonatites with initial ${ }^{87} \mathrm{Sr} /{ }^{86} \mathrm{Sr} \leqslant 0.7038, \quad{ }_{\varepsilon} \mathrm{Nd} \geqslant+1.2, \quad \varepsilon \mathrm{Hf} \geqslant+1.4, \quad$ and ${ }^{206} \mathrm{~Pb} /{ }^{204} \mathrm{~Pb} \geqslant 18.2$ (Figs. 9-11). The extremely high incompatible element concentrations of these primitive carbonate-rich rocks, along with elevated ferric/ferrous ratios, suggest that isotopically depleted oxidized melt was extracted during very low degrees of partial melting of convecting upper mantle material shortly prior to the onset of UML magmatism at ca. $610 \mathrm{Ma}$. An asthenospheric origin of the carbonate-rich material is in keeping with similar mildly depleted $\mathrm{Sr}-\mathrm{Nd}-\mathrm{Hf}$ isotope compositions of similar old aillikites/carbonatites from central Labrador and West Greenland (Nelson, 1989; Tappe et al., 2006, 2007; Gaffney et al., 2007). This indicates that the extraction of $\mathrm{CO}_{2}$-rich low-degree melts from the asthenosphere was a widely operating process beneath the NAC lithosphere during the Late Neoproterozoic. However, calculated $\mathrm{Nd}$ and $\mathrm{Hf}$ depleted mantle model ages for the isotopically depleted aillikites/ carbonatites range between 1.0 and $1.1 \mathrm{Ga}$ (Table 3), thus, indicating that an older component is likely to be involved in their genesis.

A long-term enriched isotope component, with initial ${ }^{87} \mathrm{Sr} /{ }^{86} \mathrm{Sr}>0.7038$, negative $\varepsilon \mathrm{Nd}$ and $\varepsilon \mathrm{Hf}$, and ${ }^{206} \mathrm{~Pb} /{ }^{204} \mathrm{~Pb}$ as low as 17.8, is identified in carbonate-poor Torngat mela-aillikites, but importantly, it is also present in some of the aillikites (Figs. 9-11). All of these 'enriched' samples have lower ferric/ferrous ratios than 'depleted' aillikites (Fig. 7e) and their calculated Nd- and Hf-depleted mantle model ages are slightly older (1.1-1.4 Ga). If these model ages represent mixtures of the widely recognized carbonate-rich isotopically depleted component and long-term enriched material, then the enriched 'more reduced' component can be assumed to be significantly older than $1.4 \mathrm{Ga}$.

Recycled ancient continental crust-derived material can be ruled out as the source of this enriched component given the generally high $\mathrm{Ce} / \mathrm{Pb}$ and $\mathrm{Nb} / \mathrm{U}$ of the Torngat UMLs. $\mathrm{The} \mathrm{Nb} / \mathrm{U}$ ratios correlate positively with silica and negatively with $\varepsilon \mathrm{Nd}$ (Fig. 7d); an opposite relationship would 
be expected if recycled continental crust material represents this isotopically enriched component (Hofmann et al., 1986; Carlson et al., 1996). Furthermore, it should be noted that the initial $\mathrm{Sr}-\mathrm{Nd}-\mathrm{Hf}-\mathrm{Pb}$ isotope signatures of mela-aillikites are unlike any of the time-integrated isotope compositions from crustal lithologies in the region (cf. Taylor et al., 1980; Collerson et al., 1989; Blichert-Toft et al., 1999; Vervoort and Blichert-Toft, 1999). This excludes the possibility of a relationship between aillikite and mela-aillikite magmas by assimilation of continental crust en route to the surface.

In $\mathrm{Sr}-\mathrm{Nd}-\mathrm{Hf}$ isotope space, the isotopically most enriched Torngat samples fall near to the Mesoproterozoic Aillik Bay olivine lamproites (Figs. 1, 9, and 10). Moreover, it appears that the enriched mantle component that contributed to the Torngat UML magmas has strong resemblance to the type of isotopically enriched mantle identified in many other North American primitive potassic rock suites such as the West Greenland, Leucite Hills, and Smoky Butte olivine lamproites (Fig. 9). The magma source regions of each of these potassic rock suites must have had a common history of relatively low time-integrated $\mathrm{Rb} / \mathrm{Sr}$ but extreme LREE and HFSE enrichment (low time-integrated $\mathrm{Sm} / \mathrm{Nd}$ and $\mathrm{Lu} / \mathrm{Hf}$ ), and it is generally believed that isotopic evolution of certain frozen metasomatic melts/fluids isolated within cratonic mantle lithosphere can develop such peculiar isotope signatures (Fraser et al., 1985; O'Brien et al., 1995; Davies et al., 2006; Mirnejad and Bell, 2006). There is an increasing body of evidence to support the view that minor mantle phases such as metasomatic Ti-oxides and pyroxenitic clinopyroxene are much more likely to be the principal source of negative $\varepsilon \mathrm{Hf}$ signatures than peridotitic clinopyroxene and garnet (Pearson and Nowell, 2004; Choukroun et al., 2005), which are the major hosts of Hf in the SCLM (Griffin et al., 2000). A number of studies have demonstrated that peridotitic clinopyroxene and garnet are characterized by extremely radiogenic ${ }^{176} \mathrm{Hf} /{ }^{177} \mathrm{Hf}$ typically exceeding $+10 \varepsilon \mathrm{Hf}$, even if their $\mathrm{Sr}$ and/or Nd isotope compositions indicate long-term enrichment (Schmidberger et al., 2002; Carlson et al., 2004; Ionov et al., 2005; Simon et al., 2007). Note that cratonic eclogites can also be a source of negative $\varepsilon \mathrm{Nd}$ and $\varepsilon \mathrm{Hf}$ (Jacob et al., 2005), but their trace element budget is too low to cause a significant imprint on the $\mathrm{Nd}-\mathrm{Hf}$ isotope systematics of highly incompatible element enriched magmas.

Although similar mantle source enrichment patterns with respect to the LFSE ( $\mathrm{Rb}-\mathrm{Sr})$, LREE $(\mathrm{Sm}-\mathrm{Nd})$, and HFSE ( $\mathrm{Lu}-\mathrm{Hf}$ ) can be recognized in potassic magmas erupted across Laurentia (Figs. 9 and 10), there are marked differences in terms of the $\mathrm{U} / \mathrm{Pb}$ evolution, even between neighbouring cratonic segments. This is best illustrated by the less radiogenic ${ }^{207} \mathrm{~Pb} /{ }^{204} \mathrm{~Pb}$ of the Saglek block UMLs compared to the Torngat UMLs at similar ${ }^{206} \mathrm{~Pb} /{ }^{204} \mathrm{~Pb}$ ratios (Fig. 11), but the origin of this difference is not clear to us at present. Unfortunately, we have no direct age constraints on the timing of the ancient mantle enrichment event that affected the western NAC margin before $1.4 \mathrm{Ga}$. However, inferences from other regions suggest that there was regionally widespread hydrous metasomatic overprinting of the Laurentian cratonic mantle during the
Paleo- and Mesoproterozoic (Dudas et al., 1987; Peterson and LeCheminant, 1993; Morin et al., 2005), e.g. at ca. $1.8 \mathrm{Ga}$ beneath the Wyoming craton (Carlson and Irving, 1994; Rudnick et al., 1999). This period of enhanced mantle overprinting may have been a consequence of failed alkaline magmatism beneath the newly assembled stagnant supercontinent configuration (Hoffman, 1989).

\subsection{Petrogenesis of the Torngat ultramafic lamprophyre suite}

\subsubsection{Physical appearance of deep magma source heterogeneities}

An important constraint on the location of the mantle source region comes from the thermal stability of the required source mineralogy. We have pointed out that phlogopite is essential in the melting assemblage of both aillikite and mela-aillikite magmas and this phase, along with many other of the inferred source assemblage minerals, are not stable at convecting upper mantle temperatures ( $\sim 1480{ }^{\circ} \mathrm{C}$; McKenzie and Bickle, 1988). Phlogopite is stable, however, at $P-T$ conditions of the cooler mantle lithosphere (Mengel and Green, 1989; Sweeney et al., 1993; Konzett, 1997; Ulmer and Sweeney, 2002) and this restricts aillikite and mela-aillikite melt equilibration to cratonic mantle portions $\left(<1400{ }^{\circ} \mathrm{C}\right.$; McKenzie et al., 2005). It does not, however, rule out a contribution from the convecting asthenospheric upper mantle (see previous section), e.g., in the form of metasomatizing melts/fluids.

There exists a general consensus among experimental petrologists that the generation of $\mathrm{SiO}_{2}$-poor, alkali- and volatile-rich melts cannot be produced by melting of peridotite alone (Arima and Edgar, 1983a,b; Foley, 1992a,b; Konzett, 1997; Ulmer and Sweeney, 2002; Mitchell, 2004). Recently, Foley et al. (2002) and Tappe et al. (2006) have invoked a vein-and-wall rock melting mechanism for the genesis of UML magmas similar to the model developed for the generation of lamproite melts by Foley (1992a). The veins reside in the lower reaches of the peridotite dominated cratonic SCLM and represent frozen convective mantle-derived low-degree melts produced under various oxygen fugacity and volatile conditions (cf. Green et al., 1987), which can lead to a variety of vein compositions (Foley, 1988, 1992b). Whether UML or lamproite magma is produced critically depends on the nature of the vein assemblage, i.e., phlogopite-carbonate dominated veins for aillikites as opposed to phlogopite-richterite-clinopyroxene dominated veins for lamproites. Hence, a plausible scenario for aillikite melt generation could invoke remelting of young phlogopite and carbonate plus minor apatite and Ti-oxide veins in garnet peridotite at the craton base producing potassic, hybrid carbonate - ultramafic silicate magma batches. This mechanism can account for both the thermal stability of the essential hydrous source mineralogy and the juvenile radiogenic isotope signature of aillikites since the veins originate as oxidized low-degree $\mathrm{CO}_{2}$-rich potassic silicate melts from the convective mantle shortly beforehand and, thus, largely control the incompatible element budget of the magma blend. However, the closely associated mela-aillikites fingerprint more reduced longterm isotopically enriched material within the Torngat 
UML magma source region and we have demonstrated that this component most likely resided in an old phlogopite-, K-richterite-, Ti-oxide-, and apatite-bearing assemblage. Such hydrous dominated, Ti-rich metasomatic assemblages are known from many cratons worldwide including the Wyoming and Churchill Provinces of Laurentia (Peterson and LeCheminant, 1993; Carlson and Irving, 1994; Downes et al., 2004). They form part of mantle-derived xenolith suites entrained by volatile-rich mafic/ultramafic magmas and appear to represent reaction products between hydrous alkaline melts of lamproitic composition and cratonic peridotite (Waters, 1987; Sweeney et al., 1993; Konzett et al., 1997; Grégoire et al., 2002; Downes et al., 2004). Rarely observed contact relationships indicate these assemblages form veins and striations within the deeper parts of cratonic lithosphere (Kramers et al., 1983; Waters and Erlank, 1988; Waters et al., 1989; Carlson and Irving, 1994; Rudnick et al., 1999). Among the various hydrous dominated metasomatic assemblages reported, the MARID suite of xenoliths (mica-amphibole-rutile-ilmenite-diopside; Dawson and Smith, 1977) from the Kaapvaal craton is by far the most investigated in terms of geochemistry and phase petrology. MARID nodules are highly enriched in incompatible elements (Kramers et al., 1983; Grégoire et al., 2002) and were shown to have crystallized at pressures higher than 4 GPa (Konzett, 1997; Konzett et al., 1997), i.e., within the diamond stability field. They have extreme isotopic compositions, e.g., rutiles with highly variable $\varepsilon \mathrm{Hf}$ ranging between -55 and +110 (Choukroun et al., 2005), testifying to a complex magmatic history.
In short, the above line of reasoning suggests a role for young carbonate-bearing and older hydrous dominated MARID-type vein assemblages in the genesis of the Torngat UML suite. The observed aillikite/carbonatite and mela-aillikite continuum is best explained by the concomitant fusion of these contrasting vein assemblages, which have been introduced into the deep cratonic mantle during previous melting events, but under distinctively different physicochemical conditions due to largescale structural changes within the NAC lithosphere (Fig. 13).

\subsubsection{Course of melting events}

As pointed out previously, the isotopically most enriched aillikites and mela-aillikites approach the $\mathrm{Sr}-\mathrm{Nd}-$ $\mathrm{Hf}-\mathrm{Pb}$ isotope compositions of olivine lamproites from central Labrador and West Greenland (Figs. 9-11), which were emplaced between 1400 and 1200 Ma. This Mesoproterozoic lamproite magmatism in the NAC region appears to have been confined to the craton margins (Fig. 1). Lead isotopic constraints suggest that melt/fluid-related lithospheric lamproite source enrichment occurred between ca. 2400 and 2100 Ma (Nelson, 1989; Tappe et al., 2007), i.e., during the course of the Paleoproterozoic Laurentia amalgamation. It can be inferred from our isotope data that the MARID-type vein assemblage identified within the Torngat UML magma source region was presumably also produced during this time period (>1400 Ma). This metasome, however, did not produce lamproite magma beneath the Torngat Mountains and the Saglek block during the

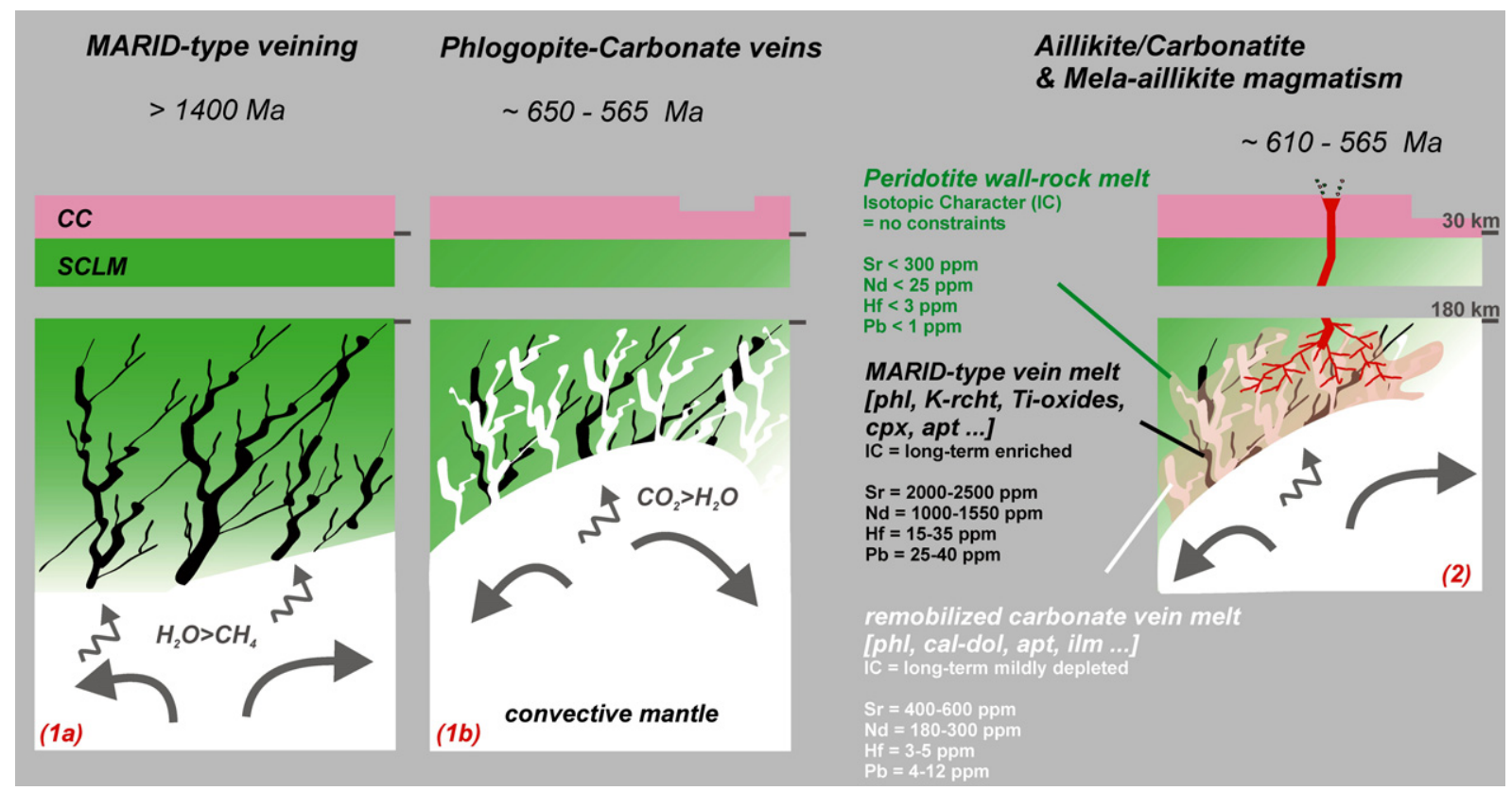

Fig. 13. Cartoon illustrating the petrogenesis of the Torngat aillikite/carbonatite and mela-aillikite compositional continuum. Important steps that may have preconditioned the magma source region and eventually led to the spectrum of hybrid UML magmas encompass: (1a) Formation of MARID-type veins within lower reaches of the NAC lithosphere before ca. $1400 \mathrm{Ma}$, presumably during the Paleoproterozoic. (1b) Injection of oxidized low-degree $\mathrm{CO}_{2}$-rich convective mantle-derived melts into this pre-existing more reduced cratonic vein network shortly prior and subsequently to ca. $610 \mathrm{Ma}$; this produced phlogopite-carbonate dominated veins. (2) Remelting of these carbonate-rich veins, which triggered volatile-fluxed melting of cratonic peridotite and the concomitant fusion of old hydrous MARID-type vein assemblages; this produced the Torngat aillikite/carbonatite and mela-aillikite melt continuum at ca. 610 and $565 \mathrm{Ma}$. 
Mesoproterozoic, presumably owing to a steeper cratonic geotherm in this particular area.

During the Late Neoproterozoic, pronounced stretching and thinning of the North Atlantic cratonic lithosphere occurred throughout contiguous Laurentia and Baltica between ca. 610 and $550 \mathrm{Ma}$ (Doig, 1970; Torsvik et al., 1996). Numerous carbonatite intrusions, many of which are associated with carbonate-rich UML magmas, were emplaced through zones of persistent lithospheric weakness such as the present-day St. Lawrence Valley and Labrador Sea rift systems (Doig and Barton, 1968; Larsen and Rex, 1992; Tappe et al., 2007). The Torngat UML magmatism occurred within this tectonic framework of incipient rifting of thick cratonic lithosphere with low-degree $\mathrm{CO}_{2}$-rich melt fractions, produced from regionally upwelling convective mantle material, continuously leaking into overlying cratonic mantle peridotite. In addition, these more oxidized $\mathrm{CO}_{2}$-rich melts may have been preferentially channelled into the pre-existing more reduced MARID-type vein network within the lower reaches of the Torngat cratonic lithosphere, where they solidified as phlogopite-carbonate dominated veins. Continued lithosphere stretching may have caused the quasi-instantaneous remelting of the low$T$ fusible carbonate-rich veins, thereby inducing volatilefluxed melting of the MARID-type veins and ambient cratonic peridotite, which eventually gave rise to the Torngat aillikite/carbonatite and mela-aillikite melt continuum at 610 to $565 \mathrm{Ma}$ (Fig. 13).

To which side of the compositional continuum a melt batch falls is a function of vein abundances, proportions, and their spatial distribution within the peridotite substrate. We view this rather complex melting scenario as a sequence of incongruent melting reactions affecting a wide range of trace element-rich solid solutions, so that pre-existing differences in the bulk incompatible element distribution between the two metasomatic assemblages will be largely masked (cf. Reiners, 1998). However, the isotopic differences observed within the Torngat UML suite provide compelling evidence for melting of a highly heterogeneous multiply metasomatized mantle source region (Fig. 13). In the following section we will test and quantify this petrogenetic model by means of the available isotope information.

\subsection{Quantitative constraints on a possible melting scenario}

Important steps that may have preconditioned the Torngat UML magma source region and eventually led to aillikite/carbonatite and mela-aillikite melt production encompass:

(1a) Formation of MARID-type veins within lower reaches of the NAC lithosphere before ca. $1400 \mathrm{Ma}$, presumably during the Paleoproterozoic; such metasomes have produced lamproite magma elsewhere in the region at ca. 1400 and $1200 \mathrm{Ma}$.

(1b) Injection of low-degree $\mathrm{CO}_{2}$-rich convective mantlederived melts into this pre-existing cratonic vein network shortly prior and subsequently to ca. $610 \mathrm{Ma}$; this produced phlogopite-carbonate dominated veins. (2a) Remelting of these carbonate-rich veins, which triggered volatile-fluxed melting of cratonic peridotite at ca. 610 and $565 \mathrm{Ma}$; this produced 'purest' aillikite melt represented by the isotopically depleted Torngat dykes and phlogopite-poor Sarfartoq dykes, West Greenland.

(2b) Concomitant fusion of the old MARID-type veins into which the $\mathrm{CO}_{2}$-rich melts were preferentially channelled (see Step 1b); this produced the Torngat aillikite/carbonatite and mela-aillikite melt continuum at ca. 610 and $565 \mathrm{Ma}$ (Fig. 13).

Whereas significant amounts of incompatible elements are pre-concentrated during Steps $1 \mathrm{a}$ and $1 \mathrm{~b}$, the high-degree partial melt produced from cratonic peridotite during Step 2a seems insignificant in terms of the incompatible element budget of the hybrid UML magmas. Hence, only the isotope compositions of the carbonate-rich and MARIDtype veins are considered in a $\mathrm{Sr}-\mathrm{Nd}-\mathrm{Hf}-\mathrm{Pb}$ isotope mixing model (Fig. 14). Unfortunately, the curvature of a potential mixing relationship between isotopically depleted and enriched materials, as suggested by the Torngat data, is not sufficient to define the involved end-member compositions by means of the mixing hyperbolae asymptotes (Figs. 911). For this reason we have performed isotope mixing calculations between plausible end-member compositions (Fig. 14; Table 4), which will be assessed along with the elemental abundances required below.

It seems reasonable to assign the isotopically depleted carbonate-rich vein component, identified in aillikites/carbonatites, to $\mathrm{CO}_{2}$-rich convective mantle-derived melts. Our compilation of high-quality $\mathrm{Sr}-\mathrm{Nd}$ isotope data from more than 500 carbonatites from over 50 worldwide occurrences shows that there is a convergence of the data array towards a cluster between 0.7029 and $0.7037{ }^{87} \mathrm{Sr} /{ }^{86} \mathrm{Sr}$ and from +2.8 to $+5.8 \varepsilon \mathrm{Nd}$ (Fig. 9). This indicates that the vast majority of worldwide carbonatites have mildly depleted isotope compositions similar to the $\mathrm{Sr}-\mathrm{Nd}$ isotope compositions of many ocean island basalts (cf. Nelson et al., 1988). However, one must be cautious when trying to model mixing relationships between melts that formed early in Earth history, i.e., at ca. 610 and $565 \mathrm{Ma}$, given the isotopic evolution of the crust-mantle system and the fact that the database is dominated by carbonatites younger than $200 \mathrm{Ma}$. Our 'depleted' end-member choice is therefore twofold: First, we use a 'mildly depleted' $\mathrm{Sr}-\mathrm{Nd}$ isotope end-member composition that falls at the unradiogenic side of the worldwide carbonatite cluster $\left(\right.$ model $\mathrm{A} ;{ }^{87} \mathrm{Sr} /{ }^{86} \mathrm{Sr}=0.7029 ; \varepsilon \mathrm{Nd}=+3$; Figs. 9, 10, 14). This rather conservative end-member choice is independently supported by similar $\mathrm{Sr}-\mathrm{Nd}$ isotope compositions of carbonatite and phlogopite-poor aillikite dykes from the Sarfartoq and Maniitsoq areas in West Greenland (Fig. 9; Nelson, 1989), which have ages within the Torngat UML range. Second, we use the most depleted $\mathrm{Nd}$ isotope composition of carbonatites yet reported $(\varepsilon \mathrm{Nd}=+7.1$; $\left.{ }^{87} \mathrm{Sr} /{ }^{86} \mathrm{Sr}=0.7030\right)$, which is viewed as a more extreme scenario (model B; Figs. 9, 10, and 14). These isotopically strongly depleted carbonatites occur in the Sokli and Kovdor complexes (Kramm, 1993; Lee et al., 2006), forming part of the ca. 380-Ma-old Kola Alkaline Province that is known 


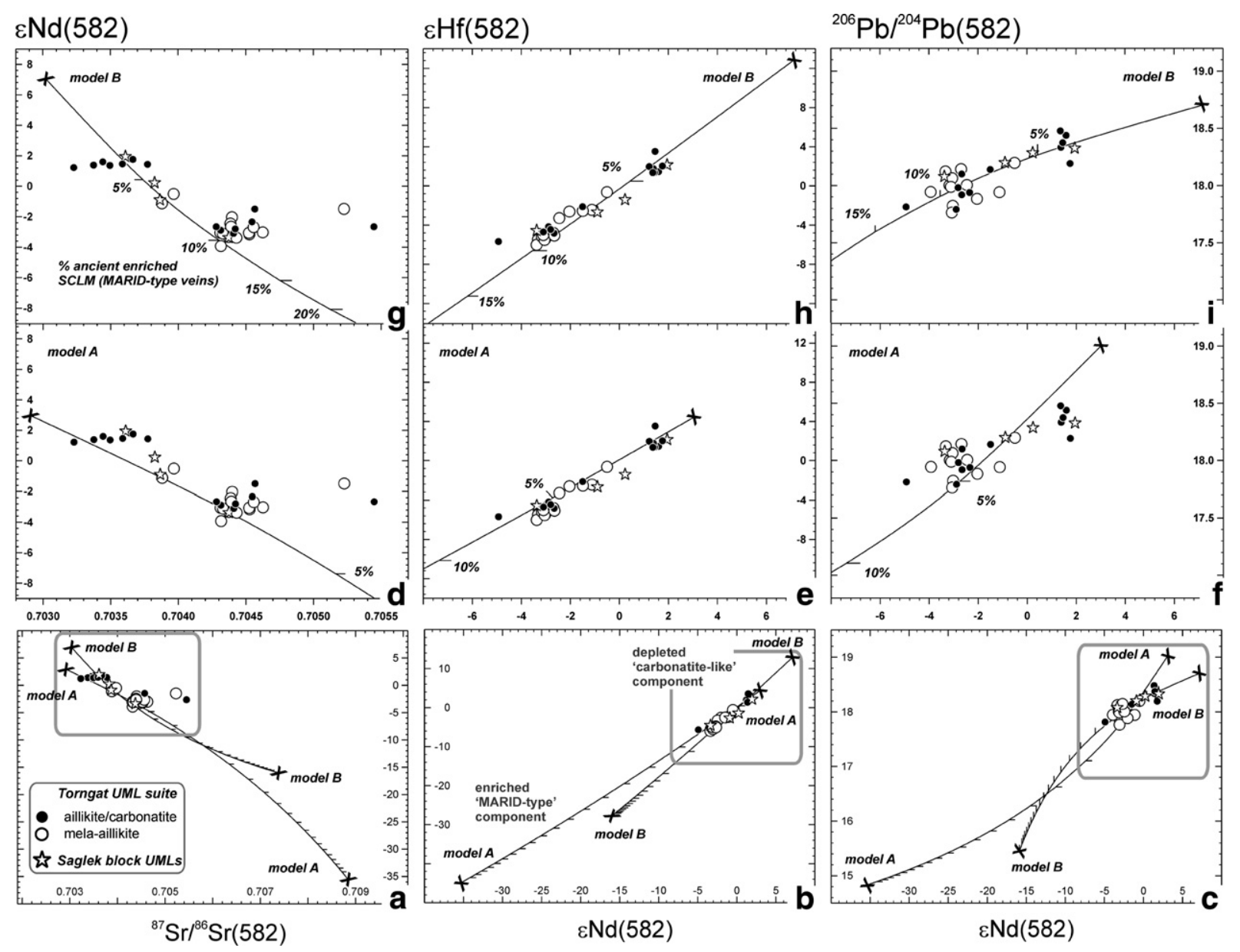

Fig. 14. Combined $\mathrm{Sr}-\mathrm{Nd}-\mathrm{Hf}-\mathrm{Pb}$ isotope variation diagrams for Torngat and Saglek block UML dykes from northern Labrador. The bottom panels (a-c) depict our binary mixing models $\boldsymbol{A}$ and $\boldsymbol{B}$ in $\mathrm{Sr}-\mathrm{Nd}, \mathrm{Nd}-\mathrm{Hf}$, and $\mathrm{Nd}-\mathrm{Pb}$ isotope space. Mixing calculations were performed between isotopically depleted 'carbonatite-like' and enriched 'MARID-type' components. Tick marks are for $5 \%$ intervals and the chosen end-member parameters are summarized in Table 4. Enlarged portions of the model $\boldsymbol{A}$ and $\boldsymbol{B}$ mixing hyperbolae are shown in the middle $(\mathrm{d}-\mathrm{f})$ and top ( $\mathrm{g}-\mathrm{i})$ panels, respectively. This modelling suggests that the incompatible element budget of the majority of aillikite/ carbonatite is dominated by a mildly depleted convective mantle-derived component. However, contribution of ca. 5-12\% incompatible elements from a long-term enriched isotopically extreme component such as MARID-type veins to the depleted carbonate-rich component can explain the compositional shift from aillikites towards mela-aillikites.

to contain UML intrusives (Beard et al., 1996; Downes et al., 2005).

Mixing hyperbolae can be fit from the two long-term depleted carbonate-rich end-members through the Torngat UML Sr-Nd isotope dataset to 1400- and 1200-Ma-old olivine lamproites from central Labrador $\left({ }^{87} \mathrm{Sr} /{ }^{86} \mathrm{Sr}=\right.$ $0.7073 ; \varepsilon \mathrm{Nd}=-15.9)$ and West Greenland $\left({ }^{87} \mathrm{Sr} /{ }^{86} \mathrm{Sr}=\right.$ 0.7088; $\varepsilon \mathrm{Nd}=-35.4)$, which have been time-integrated to $582 \mathrm{Ma}$ (Figs. 9, 14a; the $\mathrm{Rb} / \mathrm{Sr}$ ratio was corrected for $20 \%$ melting-related parent/daughter element fractionation). These lamproite isotope compositions are our best available proxy for the long-term enriched MARID-type component (Nelson, 1989; Tappe et al., 2007), because lamproitic melts were shown experimentally and geochemically to play a crucial role in MARID formation (Waters, 1987; Konzett et al., 1997). Moreover, lamproites represent the only magma type that may exceed the incompatible element concentrations of carbonatite liquids (cf. Foley et al., 1987;
Mitchell and Bergman, 1991). This is an essential prerequisite for the melt fraction that carried the isotopically enriched component, because the increase of the $\mathrm{Hf}$ concentrations towards some of the most isotopically enriched mela-aillikites (Fig. $6 \mathrm{~b}$ and c), along with pronounced $\mathrm{Sr}-\mathrm{Nd}-\mathrm{Hf}$ isotope coupling (Fig. 14a and b), requires that the enriched component contributed disproportionately large amounts of these elements to the UML magma blends. Thus, the most extreme lamproite $\mathrm{Sr}, \mathrm{Nd}$, $\mathrm{Hf}$, and $\mathrm{Pb}$ concentration level was chosen for the longterm isotopically enriched MARID-type component (cf. Mitchell and Bergman, 1991), whereas concentrations from the low end of reported carbonatites were assigned to the long-term depleted component (Table 4).

The Hf isotope composition of our 'mildly depleted' endmember (model $\mathrm{A} ; \varepsilon \mathrm{Nd}=+3 ; \varepsilon \mathrm{Hf}=+4.4$ ) was guided by carbonatite and phlogopite-poor aillikite dyke compositions from Sarfartoq and Maniitsoq, West Greenland (Bizzarro 
Table 4

$\mathrm{Sr}-\mathrm{Nd}-\mathrm{Hf}-\mathrm{Pb}$ isotope compositions and element concentrations of end-members used in the binary mixing calculations

\begin{tabular}{|c|c|c|c|c|c|c|c|c|}
\hline & \multicolumn{4}{|c|}{ Isotopically depleted component } & \multicolumn{4}{|c|}{ Isotopically enriched component } \\
\hline & ${ }^{87} \mathrm{Sr} /{ }^{86} \mathrm{Sr}$ & $\varepsilon_{\mathrm{Nd}}$ & $\varepsilon_{\mathrm{Hf}}$ & ${ }^{206} \mathrm{~Pb} /{ }^{204} \mathrm{~Pb}$ & ${ }^{87} \mathrm{Sr} /{ }^{86} \mathrm{Sr}$ & $\varepsilon_{\mathrm{Nd}}$ & $\varepsilon_{\mathrm{Hf}}$ & ${ }^{206} \mathrm{~Pb} /{ }^{204} \mathrm{~Pb}$ \\
\hline & Proxy for mild & epleted ca & atite ( & 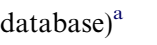 & West Greenlan & nproite \#561 & me-integra & $582 \mathrm{Ma})^{\mathrm{c}}$ \\
\hline \multirow{2}{*}{ Model A } & $0.70290(450)$ & $3.0(300)$ & $4.4(4)$ & $19.0(4)$ & $0.708829(2500)$ & $-35.4(1000)$ & $-45(15)$ & $14.83(30)$ \\
\hline & \multicolumn{4}{|c|}{ Most depleted carbonatite (Kola Alkaline Province) ${ }^{\mathrm{b}}$} & \multicolumn{4}{|c|}{ Central Labrador lamproite L68 (time-integrated to $582 \mathrm{Ma})^{\mathrm{c}}$} \\
\hline Model B & $0.703016(600)$ & $7.1(200)$ & $12.8(3)$ & $18.7(12)$ & $0.707323(2300)$ & $-15.9(1550)$ & $-27.6(35)$ & $15.45(25)$ \\
\hline
\end{tabular}

Numbers in parentheses are elemental concentrations of melts derived from end-member components in ppm. For detailed discussion of the choice of end-member compositions and further explanation see main text.

${ }^{a}$ The mildly depleted carbonatite isotope values are retrieved from our own unpublished carbonatite database (516 analyses from 50 occurrences worldwide ranging in age between Recent and $2000 \mathrm{Ma}$ ).

b The most depleted Nd isotope compositions of carbonatites are reported from the Kola Alkaline Province (i.e., Sokli and Kovdor carbonatite complexes; Kramm, 1993; Lee et al., 2006).

${ }^{c}$ Isotopic compositions of Aillik Bay (central Labrador; Tappe et al., 2007) and Sisimiut (West Greenland; Nelson, 1989) lamproites are time-integrated to $582 \mathrm{Ma}(\mathrm{Rb} / \mathrm{Sr}$ was corrected for $20 \%$ of parent/daughter element fractionation upon melting).

et al., 2002; Gaffney et al., 2007). The Hf isotope composition of the more depleted end-member (model B; $\varepsilon \mathrm{Nd}=+7.1 ; \quad \varepsilon \mathrm{Hf}=+12.8$ ) was chosen by extrapolating $\varepsilon \mathrm{Nd}$ to the terrestrial $\mathrm{Nd}-\mathrm{Hf}$ isotope array of Vervoort et al. (1999) (Figs. 10,14b). This seems reasonable in view of the general paucity of carbonatite $\mathrm{Hf}$ isotope data. The West Greenland lamproite $\varepsilon \mathrm{Hf}$ values were retrieved in a similar manner $(\varepsilon \mathrm{Nd}=-35.4 ; \varepsilon \mathrm{Hf}=-45$ at $582 \mathrm{Ma})$, whereas the Hf isotope composition of the central Labrador olivine lamproites is known $(\varepsilon \mathrm{Nd}=-15.9 ; \varepsilon \mathrm{Hf}=-27.6$ at $582 \mathrm{Ma}$; Tappe et al., 2007). The ${ }^{206} \mathrm{~Pb} /{ }^{204} \mathrm{~Pb}$ values of the two long-term depleted end-members were assigned as 19.0 for mildly depleted upper mantle (FOZO of Hauri et al., 1994) and 18.7 for the most depleted carbonatites from the Kola Peninsula (Beard et al., 1996; Lee et al., 2006). The ${ }^{206} \mathrm{~Pb} /{ }^{204} \mathrm{~Pb}$ ratios of the central Labrador and West Greenland lamproites (15.45 and 14.83 at $582 \mathrm{Ma}$, respectively) serve as a proxy for the range of $\mathrm{Pb}$ isotope compositions of old MARID-type metasomes beneath the NAC margins (Fig. 14c; Nelson, 1989; Tappe et al., 2007).

The following relationship between Torngat aillikites/ carbonatites and mela-aillikites is supported by our modelling (Fig. 14d-i; Table 4): Remelting of young phlogopitecarbonate dominated veins triggering volatile-fluxed melting of the surrounding peridotite (Step 2a) can reproduce the isotopic composition of the most depleted aillikites/carbonatites, which appear to have incorporated only very small volumes of the older isotopically enriched MARIDtype material $(\leqslant 2-5 \%)$. Our data require no more than $5-$ $12 \%$ trace element contribution from this MARID-type component to a 'pure' carbonated silicate magma blend (i.e., Sarfartoq aillikites) in order to explain the observed deflection towards more enriched $\mathrm{Sr}-\mathrm{Nd}-\mathrm{Hf}-\mathrm{Pb}$ isotope compositions as exemplified by mela-aillikites and some aillikites. This rather restricted MARID contribution to the overall Torngat UML trace element budget is in good agreement with the similar 'carbonatite-like' incompatible element patterns of aillikites/carbonatites and mela-aillikites, with only subtle variations exhibited by the latter (Fig. 5). It furthermore reinforces our suggestion that this MARID-type component is volumetrically small, albeit geochemically and isotopically extreme, and most likely represents an old cratonic vein network (Fig. 13).

\section{IMPLICATIONS FOR CRATONIC CARBONATITE AND POTASSIC MAGMATISM}

Whereas the majority of carbonate-rich magmas ultimately originate from the convective upper mantle, cratonic potassic magmas are mainly derived from metasomatized lithospheric mantle sources. This relatively straightforward scenario has emerged from experimental and isotopic studies over the past few decades. However, as each craton has a unique metasomatic history, we are left with a proliferation of primitive potassic magma compositions compared to relatively uniform convective mantle-derived magmas such as common basalts and even the less common carbonatites. Further diversification of potassic magma compositions may arise from interactions between cratonic metasomes and convective mantle-derived magmas, which presumably was the impetus for potassic magmatism in a number of alkaline rock provinces.

On the basis of new isotope data for the diamondiferous Torngat ultramafic lamprophyres, we made a case for interaction between ancient MARID-style veined lithospheric mantle, which is a commonly envisaged cratonic lamproite source, and $\mathrm{CO}_{2}$-rich melts/fluids of carbonatitic affinity invading from the convective asthenospheric mantle. The first conclusion to be drawn from our petrogenetic model is that the carbonate-rich magma production itself appears to be induced by stretching and thinning of the overlying cratonic lithosphere alone. Temperatures at the lithosphere-asthenosphere boundary must have been high enough for carbonate material to melt $\left(>1000^{\circ} \mathrm{C}\right)$, but no higher than $1250{ }^{\circ} \mathrm{C}$ (corresponding to a $40 \mathrm{mWm}^{-2}$ cratonic geotherm at ca. $180 \mathrm{~km}$ depth) to prevent the MARID-type assemblage from independent 'lamproite' melt generation, which has not occurred beneath the Torngat Mountains. Hence this implies that the ancient MARIDtype metasome has been remobilized solely by $\mathrm{CO}_{2}$-fluxing during the course of regionally widespread extension-related carbonate magmatism between ca. 610 and $550 \mathrm{Ma}$. 
This finding rules out the direct influence of a mantle plume on Late Neoproterozoic UML/carbonatite magma generation within the North Atlantic region as proposed by Tachibana et al. (2006) on noble gas isotopic grounds.

Second, it appears that the low thermal stability of carbonate-bearing assemblages under conditions of the deep cratonic mantle drastically limits the lifetime of carbonate-bearing veins compared to hydrous dominated carbonate-free vein assemblages such as MARID. This difference may help to explain the typically observed contrasting isotope signatures of otherwise similarly strong incompatible element enriched UML/carbonatite and lamproite magmas. Significant long-term radiogenic in-growth is characteristic for the hydrous metasomatic vein assemblages of anorogenic lamproites, whereas it is not facilitated within relatively short-lived carbonate-bearing vein assemblages of cratonic UML/carbonatites.

Third, we concur with Wyllie's (1989) and Eggler's (1989) notion that direct eruption of primary convective mantle-derived carbonatite may be a rare event because our model is consistent with the high potential for $\mathrm{CO}_{2}$-rich melts to interact with lithospheric mantle. For the North Atlantic craton, we suggest that apart from the initial $\mathrm{CO}_{2}$ abundances in the source region, the degree of interaction is an important factor in controlling whether $\mathrm{CO}_{2}$ (i) remains dissolved in carbonated silicate magmas, enabling separation of carbonatite intrusions at crustal levels or (ii) largely reacts out at mantle depths resulting in carbonate-poor, $\mathrm{SiO}_{2}$ undersaturated UML magmas, as in the Torngat example.

\section{ACKNOWLEDGMENTS}

This study was conducted within the DFG project Fo 181/15 (Germany) and manuscript preparation was carried out whilst S.T. was supported by the Humboldt Foundation. Randy Edmunds and Lori Dyson are thanked for logistical support during fieldwork in northern Labrador in 2003. Invitation of S.T. to fieldwork in Greenland by the Geological Survey of Denmark and Greenland in 2006 and 2007 is gratefully acknowledged as it helped to better understand alkaline and carbonatite magmatism across the North Atlantic craton. Karlis Muehlenbachs is thanked for access to his stable isotope facility and Jason Cameron, Gayle Hatchard, and Judy Schultz are thanked for advice during the geochronology work in Edmonton. The manuscript has benefited through the steering efforts of Dejan Prelevic for which we are sincerely grateful. Lotte Larsen, Hugh O'Brien, and Anton le Roex provided detailed and thoughtful reviews that improved the clarity of our argumentation, even if we were not able to address all the questions raised. This is Geological Survey of Canada Contribution 20080897 and Geocycles Publication No. 469.

\section{APPENDIX A. SUPPLEMENTARY DATA}

Supplementary data associated with this article can be found, in the online version, at doi:10.1016/j.gca.2008. 03.008.

\section{REFERENCES}

Arima M. and Edgar A. D. (1983a) High pressure experimental studies on a katungite and their bearing on the genesis of some potassium-rich magmas of the west branch of the African rift. J. Petrol. 24, 166-187.

Arima M. and Edgar A. D. (1983b) A high pressure experimental study on a magnesian-rich leucite-lamproite from the West Kimberley area, Australia: petrogenetic implications. Contrib. Mineral. Petrol. 84, 228-234.

Beard A. D., Downes H., Vetrin V. R., Kempton P. D. and Maluski H. (1996) Petrogenesis of Devonian lamprophyre and carbonatite minor intrusions, Kandalaksha Gulf (Kola Peninsula, Russia). Lithos 39, 93-119.

Bizzarro M. and Stevenson R. (2003) Major element composition of the lithospheric mantle under the North Atlantic craton: evidence from peridotite xenoliths of the Sarfartoq area, southwestern Greenland. Contrib. Mineral. Petrol. 146, 223240.

Bizzarro M., Simonetti A., Stevenson R. K. and David J. (2002) Hf isotope evidence for a hidden mantle reservoir. Geology 30, 771-774.

Blichert-Toft J., Albarede F., Rosing M. T., Frei R. and Bridgwater D. (1999) The Nd and Hf isotope evolution of the mantle through the Archean: results from the Isua supracrustals, West Greenland, and from the Birimian terranes of West Africa. Geochim. Cosmochim. Acta 63, 3901-3914.

Bridgwater D., Watson J. and Windley B. F. (1973) The Archaean craton of the North Atlantic region. Philos. Trans. Roy. Soc. Lond. 273, 493-512.

Brueckner H. K. and Rex D. C. (1980) K-Ar and Rb-Sr geochronology and $\mathrm{Sr}$ isotopic study of the Alnö alkaline complex, northeastern Sweden. Lithos 13, 111-119.

Carlson R. W. and Irving A. J. (1994) Depletion and enrichment history of subcontinental lithospheric mantle: an $\mathrm{Os}, \mathrm{Sr}, \mathrm{Nd}$ and $\mathrm{Pb}$ isotopic study of ultramafic xenoliths from the northwestern Wyoming Craton. Earth Planet. Sci. Lett. 126, 457-472.

Carlson R. W., Esperança S. and Svisero D. P. (1996) Chemical and Os isotopic study of Cretaceous potassic rocks from Southern Brazil. Contrib. Mineral. Petrol. 125, 393-405.

Carlson R. W., Irving A. J., Schulze D. J. and Hearn B. C. (2004) Timing of Precambrian melt depletion and Phanerozoic refertilization events in the lithospheric mantle of the Wyoming Craton and adjacent Central Plains Orogen. Lithos 77, 453-472.

Carlson R. W., Araujo A. L. N., Junqueira-Brod T. C., Gaspar J. C., Brod J. A., Petrinovic I. A., Hollanda M. H. B. M., Pimentel M. M. and Sichel S. (2007) Chemical and isotopic relationships between peridotite xenoliths and mafic-ultrapotassic rocks from Southern Brazil. Chem. Geol. 242, 415-434.

Chacko T., Mayeda T. K., Clayton R. N. and Goldsmith J. R. (1991) Oxygen and carbon isotope fractionations between $\mathrm{CO}_{2}$ and calcite. Geochim. Cosmochim. Acta 55, 2867-2882.

Chakhmouradian A. R. (2006) High-field-strength elements in carbonatitic rocks: geochemistry, crystal chemistry and significance for constraining the sources of carbonatites. Chem. Geol. 235, 138-160.

Choukroun M., O'Reilly S. Y., Griffin W. L., Pearson N. J. and Dawson J. B. (2005) Hf isotopes of MARID (mica-amphibolerutile-ilmenite-diopside) rutile trace metasomatic processes in the lithospheric mantle. Geology 33, 45-48.

Clarke L. B., Le Bas M. J. and Spiro B. (1994) Rare earth, trace element and stable isotope fractionation of carbonatites at Kruidfontein, Transvaal, South Africa. In Kimberlites, Related Rocks and Mantle Xenoliths (eds. H. O. A. Meyer and O. H. Leonardos). Companhia de Pesquisa de Recursos Minerais, Rio de Janeiro, Brazil, pp. 236-251.

Collerson K. D., McCulloch M. T. and Nutman A. P. (1989) Sr and $\mathrm{Nd}$ isotope systematics of polymetamorphic Archean 
gneisses from southern West Greenland and northern Labrador. Can. J. Earth Sci. 26, 446-466.

Dahlgren S. (1994) Late Proterozoic and Carboniferous ultramafic magmatism of carbonatitic affinity in Southern Norway. Lithos 31, 141-154.

Dalton J. A. and Presnall D. C. (1998) The continuum of primary carbonatitic-kimberlitic melt compositions in equilibrium with lherzolite: data from the system $\mathrm{CaO}-\mathrm{MgO}-\mathrm{Al}_{2} \mathrm{O}_{3}-\mathrm{SiO}_{2}-\mathrm{CO}_{2}$ at 6 GPa. J. Petrol. 39, 1953-1964.

Davies G. R., Stolz A. J., Mahotkin I. L., Nowell G. M. and Pearson D. G. (2006) Trace element and $\mathrm{Sr}-\mathrm{Pb}-\mathrm{Nd}-\mathrm{Hf}$ isotope evidence for ancient, fluid-dominated enrichment of the source of Aldan shield lamproites. J. Petrol. 47, 1119-1146.

Dawson J. B. and Smith J. V. (1977) The MARID (micaamphibole-rutile-ilmenite-diopside) suite of xenoliths in kimberlite. Geochim. Cosmochim. Acta 41, 309-323.

Deines P. (2002) The carbon isotope geochemistry of mantle xenoliths. Earth Sci. Rev. 58, 247-278.

Deines P. (2004) Carbon isotope effects in carbonate systems. Geochim. Cosmochim. Acta 68, 2659-2679.

Digonnet S., Goulet N., Bourne J., Stevenson R. and Archibald D. (2000) Petrology of the Abloviak aillikite dykes, New Québec: evidence for a Cambrian diamondiferous alkaline province in northeastern North America. Can. J. Earth Sci. 37, 517-533.

Doig R. (1970) An alkaline rock province linking Europe and North America. Can. J. Earth Sci. 7, 22-28.

Doig R. and Barton, Jr., J. M. (1968) Ages of carbonatites and other alkaline rocks in Quebec. Can. J. Earth Sci. 5, 1401-1407.

Downes H., MacDonald R., Upton B. G. J., Cox K. G., Bodinier J. L., Mason P. R. D., James D., Hill P. G. and Hearn, Jr., B. C. (2004) Ultramafic xenoliths from the Bearpaw Mountains, Montana, USA: evidence for multiple metasomatic events in the lithospheric mantle beneath the Wyoming Craton. J. Petrol. 45, 1631-1662.

Downes H., Balaganskaya E., Beard A. D., Liferovich R. and Demaiffe D. (2005) Petrogenetic processes in the ultramafic, alkaline and carbonatitic magmatism in the Kola Alkaline Province: a review. Lithos $\mathbf{8 5}, 48-75$.

Dudas F. O., Carlson R. W. and Eggler D. H. (1987) Regional Middle Proterozoic enrichment of the subcontinental mantle source of igneous rocks from central Montana. Geology 15, 2225.

Edgar A. D. and Vukadinovic D. (1992) Implications of experimental petrology to the evolution of ultrapotassic rocks. Lithos 28, 205-220.

Eggler D. H. (1989) Carbonatites, primary melts, and mantle dynamics. In Carbonatites: Genesis and Evolution (ed. K. Bell). Unwin Hyman, London, United Kingdom, pp. 561-579.

Eisele J., Sharma M., Galer S. J. G., Blichert-Toft J., Devey C. W. and Hofmann A. W. (2002) The role of sediment recycling in EM-1 inferred from $\mathrm{Os}, \mathrm{Pb}, \mathrm{Hf}, \mathrm{Nd}$, Sr isotope and trace element systematics of the Pitcairn Hotspot. Earth Planet. Sci. Lett. 196, 197-212.

Foley S. F. (1988) The genesis of continental basic alkaline magmas: an interpretation in terms of redox melting. J. Petrol. Special Lithosphere Issue, 139-161.

Foley S. F. (1991) High-pressure stability of the fluor- and hydroxy-endmembers of pargasite and K-richterite. Geochim. Cosmochim. Acta 55, 2689-2694.

Foley S. F. (1992a) Vein-plus-wall-rock melting mechanism in the lithosphere and the origin of potassic alkaline magmas. Lithos 28, 435-453.

Foley S. F. (1992b) Petrological characterization of the source components of potassic magmas: geochemical and experimental constraints. Lithos 28, 187-204.
Foley S. F. (1993) An experimental study of olivine lamproite: first results from the diamond stability field. Geochim. Cosmochim. Acta 57, 483-489.

Foley S. F. and Andronikov A. V. (2003) The genesis of ultramafic lamprophyres. Extended Abstracts of the Eighth International Kimberlite Conference, Victoria, Canada, CD-ROM 1-3.

Foley S. F., Taylor W. R. and Green D. H. (1986) The role of fluorine and oxygen fugacity in the genesis of the ultrapotassic rocks. Contrib. Mineral. Petrol. 94, 183-192.

Foley S. F., Venturelli G., Green D. H. and Toscani L. (1987) The ultrapotassic rocks: characteristics, classification, and constraints for petrogenetic models. Earth Sci. Rev. 24, 81-134.

Foley S. F., Jackson S. E., Fryer B. J., Greenough J. D. and Jenner G. A. (1996) Trace element partition coefficients for clinopyroxene and phlogopite in an alkaline lamprophyre from Newfoundland by LAM-ICP-MS. Geochim. Cosmochim. Acta 60, 629-638.

Foley S. F., Musselwhite D. S. and van der Laan S. R. (1999) Melt compositions from ultramafic vein assemblages in the lithospheric mantle: a comparison of cratonic and non-cratonic settings. In Proceedings of the VIIth International Kimberlite Conference (eds. J. J. Gurney, J. L. Gurney, M. D. Pascoe and S. H. Richardson). Red Roof Design, Cape Town, pp. 238-246.

Foley S. F., Barth M. G. and Jenner G. A. (2000) Rutile/melt partition coefficients for trace elements and an assessment of the influence of rutile on the trace element characteristics of subduction zone magmas. Geochim. Cosmochim. Acta 64, 933-938.

Foley S. F., Andronikov A. V. and Melzer S. (2002) Petrology of ultramafic lamprophyres from the Beaver Lake area of Eastern Antarctica and their relation to the breakup of Gondwanaland. Mineral. Petrol. 74, 361-384.

Fraser K. J., Hawkesworth C. J., Erlank A. J., Mitchell R. H. and Scott-Smith B. H. (1985) Sr, $\mathrm{Nd}$ and $\mathrm{Pb}$ isotope and minor element geochemistry of lamproites and kimberlites. Earth Planet. Sci. Lett. 76, 57-70.

Frey F. A., Green D. H. and Roy S. D. (1978) Integrated models of basalt petrogenesis: a study of quartz tholeiites to olivine melilitites from south eastern Australia utilizing geochemical and experimental petrological data. J. Petrol. 19, 463-513.

Gaffney A. M., Blichert-Toft J., Nelson B. K., Bizzarro M., Rosing M. and Albarede F. (2007) Constraints on source-forming processes of West Greenland kimberlites inferred from Hf-Nd isotope systematics. Geochim. Cosmochim. Acta 71, 2820-2836.

Gittins J., Hewins R. H. and Laurin A. F. (1975) Kimberliticcarbonatitic dikes of the Saguenay River valley, Quebec, Canada. Phys. Chem. Earth 9, 137-148.

Goldstein S. L., O'Nions R. K. and Hamilton P. J. (1984) A Sm$\mathrm{Nd}$ isotopic study of atmospheric dusts and particulates from major river systems. Earth Planet. Sci. Lett. 70, 221-236.

Green D. H. and Falloon T. J. (1998) Pyrolite: a Ringwood concept and its current expression. In The Earth's Mantle (ed. I. Jackson). Cambridge University Press, Cambridge, pp. 311378.

Green D. H. and Wallace M. E. (1988) Mantle metasomatism by ephemeral carbonatite melts. Nature 336, 459-462.

Green D. H., Falloon T. J. and Taylor W. R. (1987) Mantlederived magmas: roles of variable source peridotite and variable C-H-O fluid compositions. In Magmatic Processes: Physicochemical Principles (ed. B. O. Mysen). Geochemical Society, University Park, United States, pp. 139-154.

Greenough J. D. (1988) Minor phases in the Earth's mantle: evidence from trace- and minor-element patterns in primitive alkaline magmas. Chem. Geol. 69, 177-192.

Grégoire M., Bell D. R. and Le Roex A. P. (2002) Trace element geochemistry of phlogopite-rich mafic mantle xenoliths: their 
classification and their relationship to phlogopite-bearing peridotites and kimberlites revisited. Contrib. Mineral. Petrol. 142, 603-625.

Grégoire M., Bell D. R. and Le Roex A. P. (2003) Garnet lherzolites from the Kaapvaal Craton (South Africa): trace element evidence for a metasomatic history. J. Petrol. 44, 629657.

Griffin W. L., Pearson N. J., Belousova E. A., Jackson S. E., van Achterbergh E., O'Reilly S. Y. and Shee S. R. (2000) The Hf isotope composition of cratonic mantle: LAM-MC-ICPMS analysis of zircon megacrysts in kimberlites. Geochim. Cosmochim. Acta 64, 133-147.

Griffin W. L., O'Reilly S. Y., Doyle B. J., Pearson N. J., Coopersmith H., Kivi K., Malkovets V. G. and Pokhilenko N. P. (2004) Lithosphere mapping beneath the North American plate. Lithos 77, 873-922.

Harte B., Hunter R. H. and Kinny P. D. (1993) Melt geometry, movement and crystallization, in relation to mantle dykes, veins and metasomatism. Philos. Trans. Roy. Soc. Lond. 342, 1-21.

Hauri E. H., Whitehead J. A. and Hart S. R. (1994) Fluid dynamic and geochemical aspects of entrainment in mantle plumes. $J$. Geophys. Res. 99, 24275-24300.

Heaman L. M. (1989) The nature of the subcontinental mantle from $\mathrm{Sr}-\mathrm{Nd}-\mathrm{Pb}$ isotopic studies on kimberlitic perovskite. Earth Planet. Sci. Lett. 92, 323-334.

Heaman L. M. (2005) Patterns of kimberlite emplacement: the importance of robust geochronology. Report-Geol. Survey Denmark and Greenland 68, 25.

Heaman L. M. and Kjarsgaard B. A. (2000) Timing of eastern North American kimberlite magmatism: continental extension of the Great Meteor hotspot track? Earth Planet. Sci. Lett. 178, 253-268.

Hoffman P. F. (1988) United plates of America, the birth of a craton: early Proterozoic assembly and growth of Laurentia. Annu. Rev. Earth Planet. Sci. 16, 543-603.

Hoffman P. F. (1989) Speculations on Laurentia's first gigayear (2.0 to $1.0 \mathrm{Ga})$. Geology 17, 135-138.

Hoffman P. F. (1990) Dynamics of the tectonic assembly of northeast Laurentia in geon $18(1.9-1.8 \mathrm{Ga})$. Geosci. Can. 17, 222-226.

Hofmann A. W., Jochum K. P., Seufert M. and White W. M. (1986) $\mathrm{Nb}$ and $\mathrm{Pb}$ in oceanic basalts: new constraints on mantle evolution. Earth Planet. Sci. Lett. 79, 33-45.

Ionov D. A. and Hofmann A. W. (1995) Nb-Ta-rich mantle amphiboles and micas: implications for subduction-related metasomatic trace element fractionations. Earth Planet. Sci. Lett. 131, 341-356.

Ionov D. A., Blichert-Toft J. and Weis D. (2005) Hf isotope compositions and HREE variations in off-craton garnet and spinel peridotite xenoliths from central Asia. Geochim. Cosmochim. Acta 69, 2399-2418.

Irving A. J. (1980) Petrology and geochemistry of composite ultramafic xenoliths in alkalic basalts and implications for magmatic processes within the mantle. Am. J. Sci. 280, 389426.

Jacob D. E., Bizimis M. and Salters V. J. M. (2005) Lu/Hf and geochemical systematics of recycled ancient oceanic crust: evidence from Roberts Victor eclogites. Contrib. Mineral. Petrol. 148, 707-720.

Jaffey A. H., Flynn K. F., Glendenin L. E., Bentley W. C. and Essling A. M. (1971) Precision measurements of half-lives and specific activities of ${ }^{235} \mathrm{U}$ and ${ }^{238} \mathrm{U}$. Phys. Rev. 4, 1889-1906.

Keller J. and Hoefs J. (1995) Stable isotope characteristics of recent natrocarbonatite from Oldoinyo Lengai. In Carbonatite Volcanism: Oldoinyo Lengai and the Petrogenesis of Natrocarbonatites (eds. K. Keller and J. Bell). Springer, Berlin, pp. 113-123.
Kirkley M. B., Smith H. S. and Gurney J. J. (1989) Kimberlite carbonates: a carbon and oxygen stable isotope study. In Kimberlites and Related Rocks (ed. J. Ross). Geological Society of Australia, Sydney, NSW, Australia, pp. 264-281.

Klemme S. and Dalpe C. (2003) Trace-element partitioning between apatite and carbonatite melt. Am. Miner. 88, 639-646.

Klemme S., Prowatke S., Hametner K. and Günther D. (2005) Partitioning of trace elements between rutile and silicate melts: implications for subduction zones. Geochim. Cosmochim. Acta 69, 2361-2371.

Klemme S., Günther D., Hametner K., Prowatke S. and Zack T. (2006) The partitioning of trace elements between ilmenite, ulvospinel, annalcolite and silicate melts with implications for the early differentiation of the moon. Chem. Geol. 234, 251-263.

Konzett J. (1997) Phase relations and chemistry of Ti-rich Krichterite-bearing mantle assemblages: an experimental study to 8.0 GPa in a Ti-KNCMASH system. Contrib. Mineral. Petrol. 128, 385-404.

Konzett J. and Ulmer P. (1999) The stability of hydrous potassic phases in lherzolite mantle: an experimental study to $9.5 \mathrm{GPa}$ in simplified and natural bulk compositions. J. Petrol. 40, 629652.

Konzett J., Sweeney R. J., Thompson A. B. and Ulmer P. (1997) Potassium amphibole stability in the upper mantle: an experimental study in a peralkaline KNCMASH system to $8.5 \mathrm{GPa}$. J. Petrol. 38, 537-568.

Korstgård J., Ryan B. and Wardle R. J. (1987) The boundary between Proterozoic and Archaean crustal blocks in central West Greenland and northern Labrador. In Evolution of the Lewisian and Comparable Precambrian High Grade Terrains (eds. R. G. Park and J. Tarney). Geological Society of London, London, United Kingdom, pp. 247-259.

Kramers J. D., Roddick J. C. M. and Dawson J. B. (1983) Trace element and isotope studies on veined, metasomatic and "MARID" xenoliths from Bultfontein, South Africa. Earth Planet. Sci. Lett. 65, 90-106.

Kramm U. (1993) Mantle components of carbonatites from the Kola alkaline province, Russia and Finland: a Nd-Sr study. Eur. J. Mineral. 5, 985-989.

Larsen L. M. and Rex D. C. (1992) A review of the 2500 Ma span of alkaline-ultramafic, potassic and carbonatitic magmatism in West Greenland. Lithos 28, 367-402.

Lee M. J., Lee J. I., Do Hur S., Kim Y., Moutte J. and Balaganskaya E. (2006) Sr-Nd-Pb isotopic compositions of the Kovdor phoscorite-carbonatite complex, Kola Peninsula, NW Russia. Lithos 91, 250-261.

Lugmair G. W. and Marti K. (1978) Lunar initial ${ }^{143} \mathrm{Nd} /{ }^{144} \mathrm{Nd}$ : differential evolution of the lunar crust and mantle. Earth Planet. Sci. Lett. 39, 349-357.

Mattey D. P., Lowry D. and Macpherson C. (1994) Oxygen isotope composition of mantle peridotite. Earth Planet. Sci. Lett. 128, 231-241.

McKenzie D. and Bickle M. J. (1988) The volume and composition of melt generated by extension of the lithosphere. J. Petrol. 29, 625-679.

McKenzie D., Jackson J. and Priestley K. (2005) Thermal structure of oceanic and continental lithosphere. Earth Planet. Sci. Lett. 233, 337-349.

Meen J. K., Ayers J. C. and Fregeau E. J. (1989) A model of mantle metasomatism by carbonated alkaline melts: trace-element and isotopic compositions of mantle source regions of carbonatite and other continental igneous rocks. In Carbonatites: Genesis and Evolution (ed. K. Bell). Unwin Hyman, London, United Kingdom, pp. 464-499.

Mengel K. and Green D. H. (1989) Stability of amphibole and phlogopite in metasomatized peridotite under water-saturated 
and water-undersaturated conditions. In Kimberlites and Related Rocks (ed. J. Ross). Geological Society of Australia, Sydney, NSW, Australia, pp. 571-581.

Menzies M. A. (1983) Mantle ultramafic xenoliths in alkaline magmas: evidence for mantle heterogeneity modified by magmatic activity. In Continental Basalts and Mantle Xenoliths (eds. C. J. Hawkesworth and M. J. Norry). Shiva, Nantwich, pp. 92110.

Michard A., Gurriet P., Soudant M. and Albarede F. (1985) Nd isotopes in French Phanerozoic shales: external versus internal aspects of crustal evolution. Geochim. Cosmochim. Acta 49, 601-610.

Mirnejad H. and Bell K. (2006) Origin and source evolution of the Leucite Hills lamproites: evidence from $\mathrm{Sr}-\mathrm{Nd}-\mathrm{Pb}-\mathrm{O}$ isotopic compositions. J. Petrol. 47, 2463-2489.

Mitchell R. H. (1995) Melting experiments on a sanidine phlogopite lamproite at 4-7 GPa and their bearing on the sources of lamproitic magmas. J. Petrol. 36, 1455-1474.

Mitchell R. H. (2004) Experimental studies at 5-12 GPa of the Ondermatjie hypabyssal kimberlite. Lithos 76, 551-564.

Mitchell R. H. (2005) Carbonatites and carbonatites and carbonatites. Can. Miner. 43, 2049-2068.

Mitchell R. H. and Bergman S. C. (1991) Petrology of Lamproites. Plenum Press, New York.

Mitchell R. H., Scott-Smith B. H. and Larsen L. M. (1999) Mineralogy of ultramafic dikes from the Sarfartoq, Sisimiut and Maniitsoq areas, West Greenland. In Proceedings of the VIIth international kimberlite conference (eds. J. J. Gurney, J. L. Gurney, M. D. Pascoe and S. H. Richardson). Red Roof Design, Cape Town, pp. 574-583.

Morin D., Hébert R. and Corriveau L. (2005) Mesoproterozoic deep K-magmatism recorded in a megacryst- and xenolithbearing minette dyke, western Grenville Province. Can. J. Earth Sci. 42, 1881-1907.

Münker C., Weyer S., Scherer E. and Mezger K. (2001) Separation of high field strength elements ( $\mathrm{Nb}, \mathrm{Ta}, \mathrm{Zr}, \mathrm{Hf}$ ) and $\mathrm{Lu}$ from rock samples for MC-ICPMS measurements. Geochem. Geophys. Geosyst. 2, $2001 \mathrm{GC} 000183$.

Nelson D. R. (1989) Isotopic characteristics and petrogenesis of the lamproites and kimberlites of central West Greenland. Lithos 22, 265-274.

Nelson D. R., Chivas A. R., Chappell B. W. and McCulloch M. T. (1988) Geochemical and isotopic systematics in carbonatites and implications for the evolution of ocean-island sources. Geochim. Cosmochim. Acta 52, 1-17.

Nielsen T. F. D. and Jensen S. M. (2005) The Majuagaa calcite-kimberlite dyke, Maniitsoq, southern West Greenland. Report-Geol. Survey Denmark and Greenland 43, 1-59.

Nowell G. M., Pearson D. G., Bell D. R., Carlson R. W., Smith C. B., Kempton P. D. and Noble S. R. (2004) Hf isotope systematics of kimberlites and their megacrysts: new constraints on their source regions. J. Petrol. 45, 1583-1612.

O'Brien H. E., Irving A. J., McCallum I. S. and Thirlwall M. F. (1995) Strontium, neodymium, and lead isotope evidence for the interaction of post-subduction asthenospheric potassic mafic magmas of the Highwood Mountains, Montana, USA, with ancient Wyoming craton lithospheric mantle. Geochim. Cosmochim. Acta 59, 4539-4556.

Olafsson M. and Eggler D. H. (1983) Phase relations of amphibole, amphibole-carbonate, and phlogopite-carbonate peridotite: petrologic constraints on the asthenosphere. Earth Planet. Sci. Lett. 64, 305-315.

O'Reilly S. Y. and Griffin W. L. (2000) Apatite in the mantle: implications for metasomatic processes and high heat production in Phanerozoic mantle. Lithos 53, 217-232.
Palme H. and O'Neill H. S. C. (2003) Cosmochemical estimates of mantle composition. In Treatise on Geochemistry (ed. R. W. Carlson). Elsevier, Amsterdam, pp. 1-38.

Patchett P. J., Vervoort J. D., Söderlund U. and Salters V. J. M. (2004) $\mathrm{Lu}-\mathrm{Hf}$ and $\mathrm{Sm}-\mathrm{Nd}$ isotopic systematics in chondrites and their constraints on the Lu-Hf properties of the Earth. Earth Planet. Sci. Lett. 222, 29-41.

Pearson D. G. and Nowell G. M. (2004) Re-Os and Lu-Hf isotope constraints on the origin and age of pyroxenites from the Beni Bousera peridotite massif: implications for mixed peridotitepyroxenite mantle sources. J. Petrol. 45, 439-455.

Peterson T. D. and LeCheminant A. N. (1993) Glimmerite xenoliths in early Proterozoic ultrapotassic rocks from the Churchill Province. Can. Miner. 31, 801-819.

Peterson T. D., Esperanca S. and LeCheminant A. N. (1994) Geochemistry and origin of the Proterozoic ultrapotassic rocks of the Churchill Province, Canada. Mineral. Petrol. 51, 251276.

Peucat J. J., Vidal P., Bernard-Griffiths J. and Condie K. C. (1988) $\mathrm{Sr}, \mathrm{Nd}$, and $\mathrm{Pb}$ isotopic systematics in the Archean low- to high-grade transition zone of southern India: syn-accretion versus post-accretion granulites. J. Geol. 97, 537-550.

Pfänder J. A., Münker C., Stracke A. and Mezger K. (2007) Nb/Ta and $\mathrm{Zr} / \mathrm{Hf}$ in ocean island basalts-implications for crustmantle differentiation and the fate of Niobium. Earth Planet. Sci. Lett. 254, 158-172.

Ray J. S. and Ramesh R. (2000) Rayleigh fractionation of stable isotopes from a multicomponent source. Geochim. Cosmochim. Acta 64, 299-306.

Reiners P. W. (1998) Reactive melt transport in the mantle and geochemical signatures of mantle-derived magmas. J. Petrol. 39, 1039-1061.

Rivers T., Mengel F., Scott D. J., Campbell L. M. and Goulet N. (1996) Torngat Orogen - a Palaeoproterozoic example of a narrow doubly vergent collisional orogen. In Precambrian Crustal Evolution in the North Atlantic Region (ed. T. S. Brewer). Geological Society, London, United Kingdom, pp. 117-136.

Rock N. M. S. (1991) Lamprophyres. Blackie \& Son, Glasgow.

Rogers N. W., Hawkesworth C. J. and Palacz Z. A. (1992) Phlogopite in the generation of olivine melilitites from Namaqualand, South Africa and implications for element fractionation processes in the upper mantle. Lithos 28, 347365.

Romer R. L., Heinrich W., Schroder-Smeibidl B., Meixner A., Fischer C. O. and Schulz C. (2005) Elemental dispersion and stable isotope fractionation during reactive fluid-flow and fluid immiscibility in the Bufa del Diente aureole, NE-Mexico: evidence from radiographies and $\mathrm{Li}, \mathrm{B}, \mathrm{Sr}, \mathrm{Nd}$, and $\mathrm{Pb}$ isotope systematics. Contrib. Mineral. Petrol. 149, 400-429.

Rudnick R. L. and Fountain D. M. (1995) Nature and composition of the continental crust: a lower crustal perspective. Rev. Geophys. 33, 267-309.

Rudnick R. L., Ireland T. R., Gehrels G., Irving A. J., Chesley J. T. and Hanchar J. M. (1999) Dating mantle metasomatism: U-Pb geochronology of zircons in cratonic mantle xenoliths from Montana and Tanzania. In Proceedings of the VIIth International Kimberlite Conference (eds. J. J. Gurney, J. L. Gurney, M. D. Pascoe and S. H. Richardson). Red Roof Design, Cape Town, pp. 728-735.

Salters V. J. M. and Hart S. R. (1991) The mantle sources of ocean ridges, islands and arcs: the Hf-isotope connection. Earth Planet. Sci. Lett. 104, 364-380.

Santos R. V. and Clayton R. N. (1995) Variations of oxygen and carbon isotopes in carbonatites: a study of Brazilian alkaline complexes. Geochim. Cosmochim. Acta 59, 1339-1352. 
Scherer E., Münker C. and Mezger K. (2001) Calibration of the Lutetium-Hafnium clock. Science 293, 683-687.

Schmidberger S. S., Simonetti A., Francis D. and Gariépy C. (2002) Probing Archean lithosphere using the $\mathrm{Lu}-\mathrm{Hf}$ isotope systematics of peridotite xenoliths from Somerset Island kimberlites, Canada. Earth Planet. Sci. Lett. 197, 245-259.

Simon N. S. C., Carlson R. W., Pearson D. G. and Davies G. R. (2007) The origin and evolution of the Kaapvaal cratonic lithospheric mantle. J. Petrol. 48, 589-625.

Stacey J. S. and Kramers J. D. (1975) Approximation of terrestrial lead isotope evolution by a two-stage model. Earth Planet. Sci. Lett. 26, 207-221.

Steiger R. H. and Jäger E. (1977) Subcommission on geochronology: convention on the use of decay constants in geo- and cosmochronology. Earth Planet. Sci. Lett. 36, 359-362.

Sudo A. and Tatsumi Y. (1990) Phlogopite and K-amphibole in the upper mantle: implication for magma genesis in subduction zones. Geophys. Res. Lett. 17, 29-32.

Sweeney R. J., Thompson A. B. and Ulmer P. (1993) Phase relations of a natural MARID composition and implications for MARID genesis, lithospheric melting and mantle metasomatism. Contrib. Mineral. Petrol. 115, 225-241.

Tachibana Y., Kaneoka I., Gaffney A. and Upton B. G. J. (2006) Ocean-island basalt-like source of kimberlite magmas from West Greenland revealed by high ${ }^{3} \mathrm{He} /{ }^{4} \mathrm{He}$ ratios. Geology $\mathbf{3 4}$, 273-276.

Tappe S., Jenner G. A., Foley S. F., Heaman L. M., Besserer D., Kjarsgaard B. A. and Ryan B. (2004) Torngat ultramafic lamprophyres and their relation to the North Atlantic Alkaline Province. Lithos 76, 491-518.

Tappe S., Foley S. F., Jenner G. A. and Kjarsgaard B. A. (2005) Integrating ultramafic lamprophyres into the IUGS classification of igneous rocks: rational and implications. J. Petrol. 46, 1893-1900.

Tappe S., Foley S. F., Jenner G. A., Heaman L. M., Kjarsgaard B. A., Romer R. L., Stracke A., Joyce N. and Hoefs J. (2006) Genesis of ultramafic lamprophyres and carbonatites at Aillik Bay, Labrador: a consequence of incipient lithospheric thinning beneath the North Atlantic craton. J. Petrol. 47, 1261-1315.

Tappe S., Foley S. F., Stracke A., Romer R. L., Kjarsgaard B. A., Heaman L. M. and Joyce N. (2007) Craton reactivation on the Labrador Sea margins: ${ }^{40} \mathrm{Ar} /{ }^{39} \mathrm{Ar}$ age and $\mathrm{Sr}-\mathrm{Nd}-\mathrm{Hf}-\mathrm{Pb}$ isotope constraints from alkaline and carbonatite intrusives. Earth Planet. Sci. Lett. 256, 433-454.

Taylor P. N., Moorbath S., Goodwin R. and Petrykowski A. C. (1980) Crustal contamination as an indicator of the extent of early Archaean continental crust: $\mathrm{Pb}$ isotopic evidence from the late Archaean gneisses of West Greenland. Geochim. Cosmochim. Acta 44, 1437-1454.

Thibault Y., Edgar A. D. and Lloyd F. E. (1992) Experimental investigation of melts from a carbonated phlogopite lherzolite: implications for metasomatism in the continental lithospheric mantle. Am. Miner. 77, 784-794.
Tiepolo M., Zanetti A., Oberti R., Brumm R., Foley S. F. and Vannucci R. (2003) Trace-element partitioning between synthetic potassic-richterites and silicate melts, and contrasts with the partitioning behaviour of pargasites and kaersutites. Eur. J. Mineral. 15, 329-340.

Torsvik T. H., Smethurst M. A., Meert J. G., Van der Voo R., McKerrow W. S., Brasier M. D., Sturt B. A. and Walderhaug H. J. (1996) Continental break-up and collision in the Neoproterozoic and Palaeozoic: a tale of Baltica and Laurentia. Earth Sci. Rev. 40, 229-258.

Ulmer P. and Sweeney R. J. (2002) Generation and differentiation of Group II kimberlites: constraints from a high-pressure experimental study to $10 \mathrm{GPa}$. Geochim. Cosmochim. Acta 66, 2139-2153.

Vervoort J. D. and Blichert-Toft J. (1999) Evolution of the depleted mantle: Hf isotope evidence from juvenile rocks through time. Geochim. Cosmochim. Acta 63, 533-556.

Vervoort J. D., Patchett P. J., Blichert-Toft J. and Albarede F. (1999) Relationships between $\mathrm{Lu}-\mathrm{Hf}$ and $\mathrm{Sm}-\mathrm{Nd}$ isotopic systems in the global sedimentary system. Earth Planet. Sci. Lett. 168, 79-99.

Wardle R. J., James D. T., Scott D. J. and Hall J. (2002) The southeastern Churchill Province: synthesis of a Paleoproterozoic transpressional orogen. Can. J. Earth Sci. 39, 639663.

Waters F. G. (1987) A suggested origin of MARID xenoliths in kimberlites by high pressure crystallization of an ultrapotassic rock such as lamproite. Contrib. Mineral. Petrol. 95, 523-533.

Waters F. G. and Erlank A. J. (1988) Assessment of the vertical extent and distribution of mantle metasomatism below Kimberley, South Africa. J. Petrol. Special Lithosphere Issue, 185204.

Waters F. G., Erlank A. J. and Daniels L. R. M. (1989) Contact relationships between MARID rock and metasomatised peridotite in a kimberlite xenolith. Geochem. J. 23, 11-17.

Watson E. B. and Green T. H. (1981) Apatite/ liquid partition coefficients for the rare earth elements and strontium. Earth Planet. Sci. Lett. 56, 405-421.

Wilson M. R., Kjarsgaard B. A. and Taylor B. (2007) Stable isotope composition of magmatic and deuteric carbonate phases in hypabyssal kimberlite, Lac de Gras field, Northwest Territories, Canada. Chem. Geol. 242, 435-454.

Wyllie P. J. (1989) Origin of carbonatites: evidence from phase equilibrium studies. In Carbonatites: Genesis and Evolution (ed. K. Bell). Unwin Hyman, London, United Kingdom, pp. 500545.

Wyllie P. J. and Huang W. L. (1975) Influence of mantle $\mathrm{CO}_{2}$ in the generation of carbonatites and kimberlites. Nature 257, 297299.

Associate editor: Martin A. Menzies 